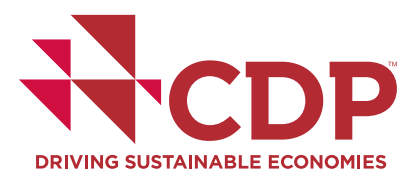

\title{
CDP Russia Climate Change Report 2014
}

\section{On behalf of 767 investors with assets of US\$92 trillion}

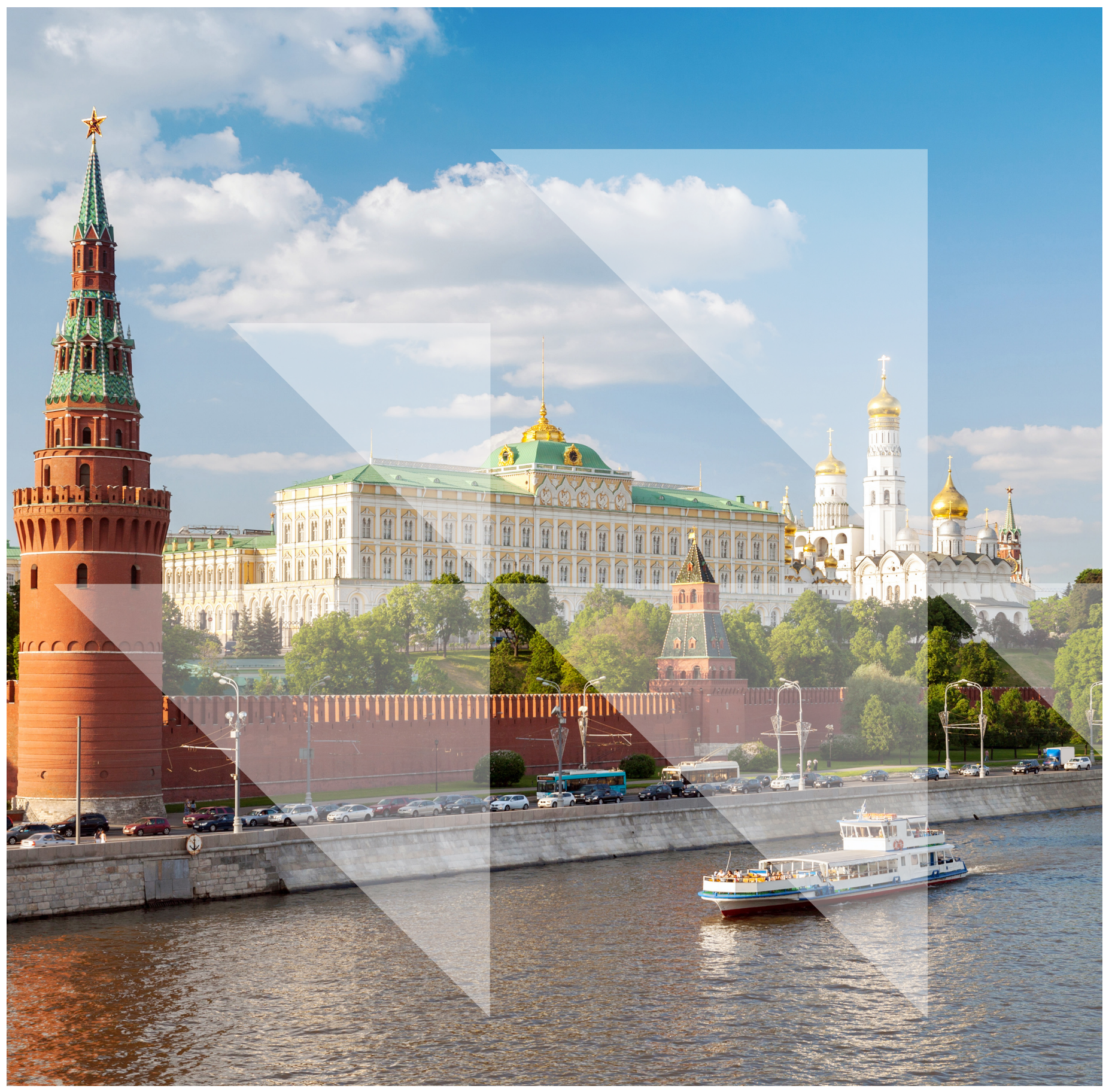




\section{Investor members}

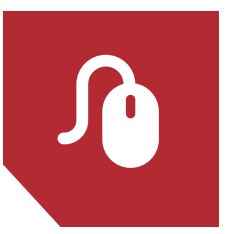

CDP works with investors globally to advance the investment opportunities and reduce the risks posed by climate change by asking over 5,000 of the world's largest companies to report their climate strategies, GHG emissions and energy use through CDP's standardized format. To learn more about CDP's member offering and becoming a member, please contact us or visit www.cdp.net/en-US/WhatWeDo/.

\section{Where are the signatory investors located?*}
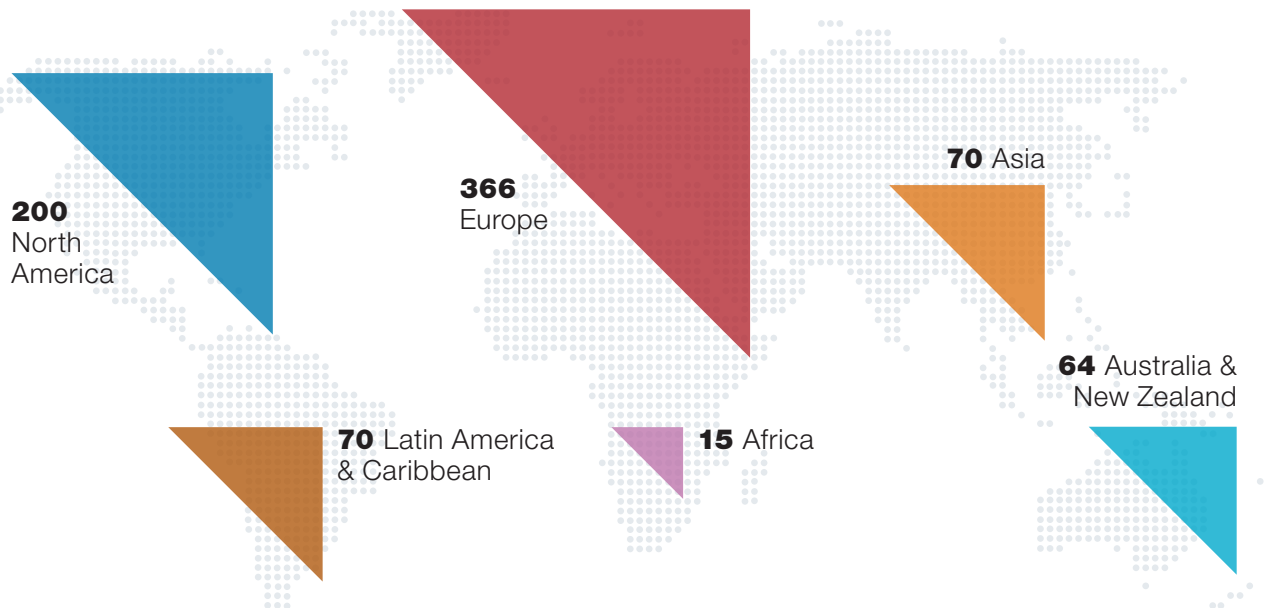

\section{CDP investor base continues to grow*}

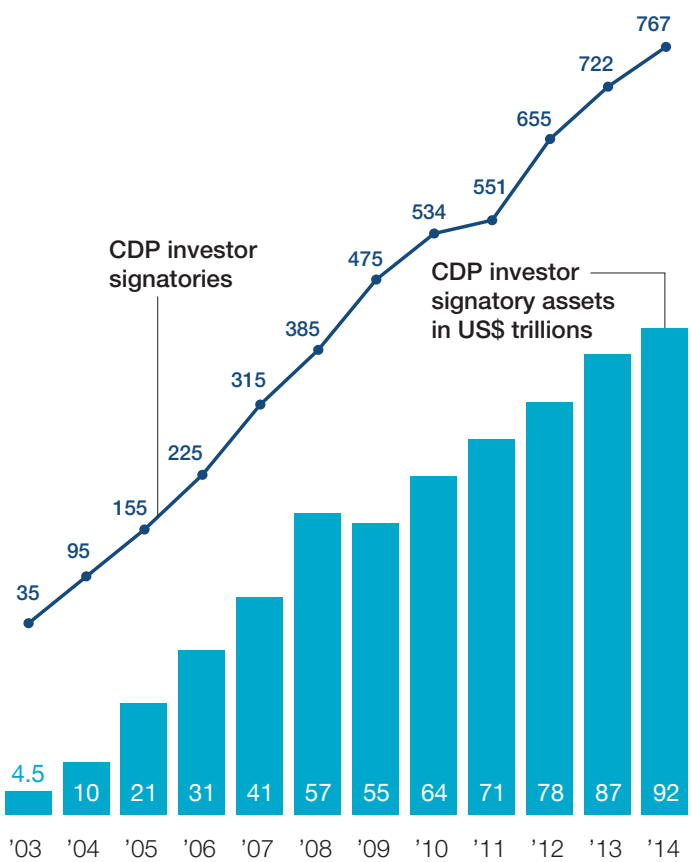

\section{Investors by type}

312 Asset managers

256 Asset owners

152 Banks

38 Insurance

27 Other

\section{CDP investor members 2014}

ABRAPP_Associação Brasileira das Entidades Fechadas de Previdência Complementar

AEGON N.V.

ATP Group

$\overline{\text { Aviva plc }}$

Aviva Investors

Bank of America Merrill Lynch

Bendigo \& Adelaide Bank Limited

BlackRock

Boston Common Asset Management, LLC

BP Investment Management Limited

California Public Employees'

Retirement System

California State Teachers' Retirement System

Calvert Investment Management, Inc.

Capricorn Investment Group, LLC

Catholic Super

CCLA Investment Management Ltd

ClearBridge Investments

DEXUS Property Group

$\overline{\text { Fachesf }}$

Fapes

Fundação Itaú Unibanco

Generation Investment Management

Goldman Sachs Group Inc.

Henderson Global Investors

HSBC Holdings plc

$\overline{\text { Infraprev }}$

$\overline{\mathrm{KLP}}$

Legg Mason Global Asset Management

London Pensions Fund Authority

$\overline{\text { Mobimo Holding AG }}$

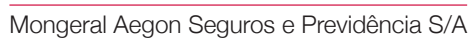

Morgan Stanley

National Australia Bank Limited

Neuberger Berman

Nordea Investment Management

Norges Bank Investment Management

NEI Investments

Petros

$\overline{\text { PFA Pension }}$

$\overline{\text { Previ }}$

$\overline{\text { Real Grandeza }}$

Robeco

RobecoSAM AG

Rockefeller Asset Management, Sustainability \& Impact Investing Group

Royal Bank of Canada

Royal Bank of Scotland Group

Sampension KP Livsforsikring A/S

Schroders

Scottish Widows Investment Partnership

$\overline{\text { SEB AB }}$

$\overline{\text { Serpros }}$

Sistel

Sompo Japan Nipponkoa Holdings, Inc

Standard Chartered

TD Asset Management

The Wellcome Trust 


\section{Contents}

Investor members

CDP foreword - Paul Simpson, CEO

Introduction - Melsa Ararat, Director, CDP Turkey

EY Russia commentary 

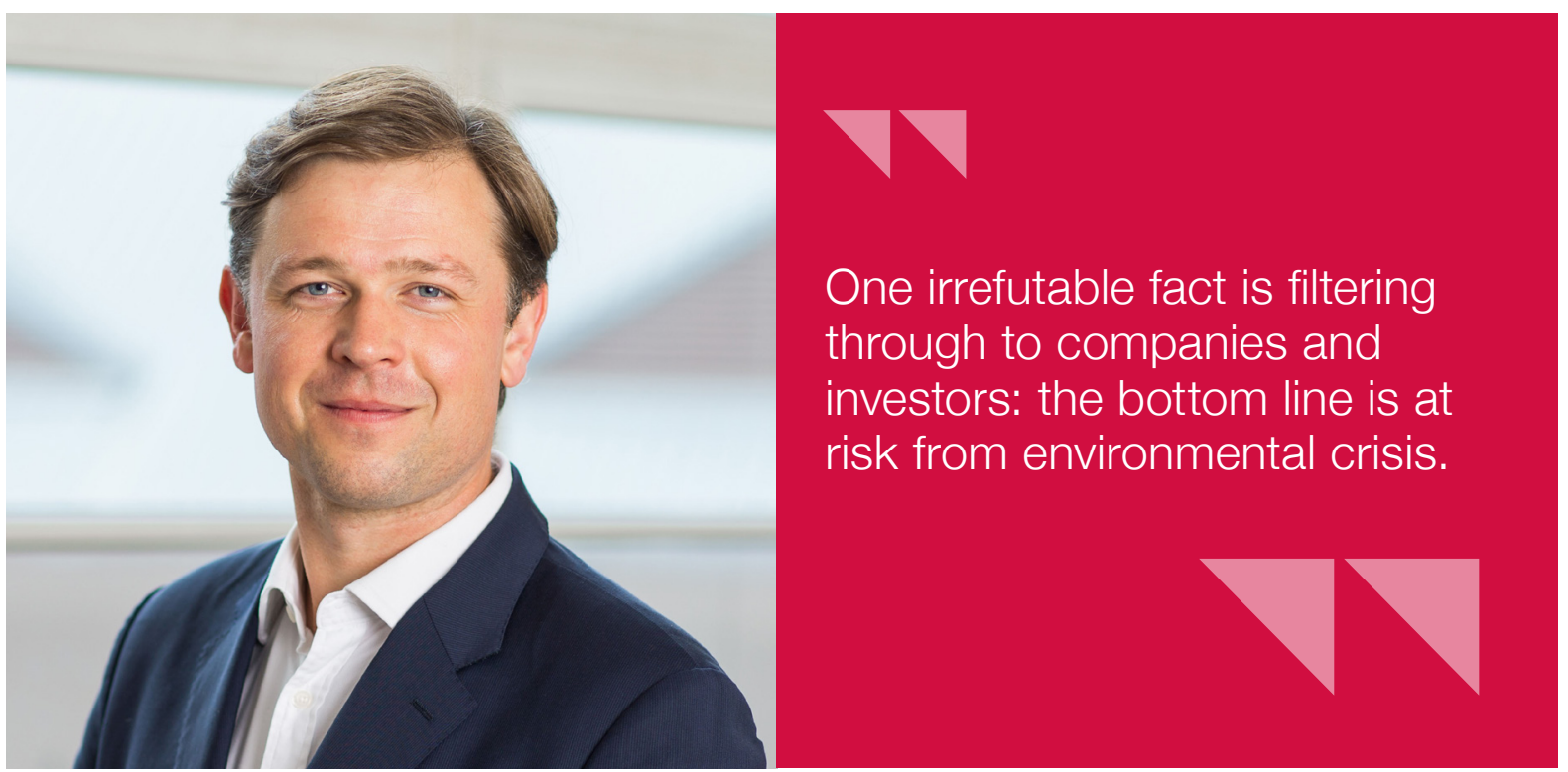

\section{The global economy has bounced back from crisis and a cautious optimism is beginning to pervade the markets. As we embrace recovery we must remember that greenhouse gas emissions continue to rise and we face steep financial risk if we do not mitigate them.}

The unprecedented environmental challenges that we confront today-reducing greenhouse gas emissions, safeguarding water resources and preventing the destruction of forests-are also economic problems. One irrefutable fact is filtering through to companies and investors: the bottom line is at risk from environmental crisis.

The impact of climate events on economies around the world has increasingly been splashed across headlines in the last year, with the worst winter in 30 years suffered by the USA costing billions of dollars. Australia has experienced its hottest two years on record and the UK has had its wettest winter for hundreds of years costing the insurance industry over a billion pounds. Over three quarters of companies reporting to CDP this year have disclosed a physical risk from climate change. Investing in climate change-related resilience planning has become crucial for all corporations.

Investor engagement on these issues is increasing. In the US a record number of shareholder resolutions in the 2014 proxy season led 20 international corporations to commit to reduce greenhouse gas emissions or sustainably source palm oil.

As mainstream investors begin to recognize the real value at risk, we are seeing more action from some of the 767 investors who request disclosure through CDP. The Norwegian pension fund, Norges Bank, with assets worth over $\$ 800$ billion, expects companies to show strategies for climate change risk mitigation and water management, and have divested from both timber and palm oil companies that did not meet their standards.
There is growing momentum on the policy front with President Obama's announcement of new federal rules to limit greenhouse gases in the US. In the EU, some 6,000 companies will be required to disclose on specific environmental, social and governance criteria as part of their mainstream reporting to investors. In China over 20,000 companies will be required to report their greenhouse gas emissions to the government.

There is a palpable sea change in approach by companies driven by a growing recognition that there is a cost associated with the carbon they emit. Measurement, transparency and accountability drives positive change in the world of business and investment. Our experience working with over 4,500 companies shows the multitude of benefits for companies that report their environmental impacts, unveiling risks and previously unseen opportunities.

We are standing at a juncture in history. With the prospect of a global climate deal ${ }^{1}$ coming from the United Nations process, governments, cities, the private sector and civil society have a great opportunity to take bold actions and build momentum in the run up to the Paris 2015 meeting. The decisions we make today can lead us to a profitable and secure future. A future that we can all be proud of.

\section{Paul Simpson}

Chief Executive Officer, CDP 


\section{Introduction}

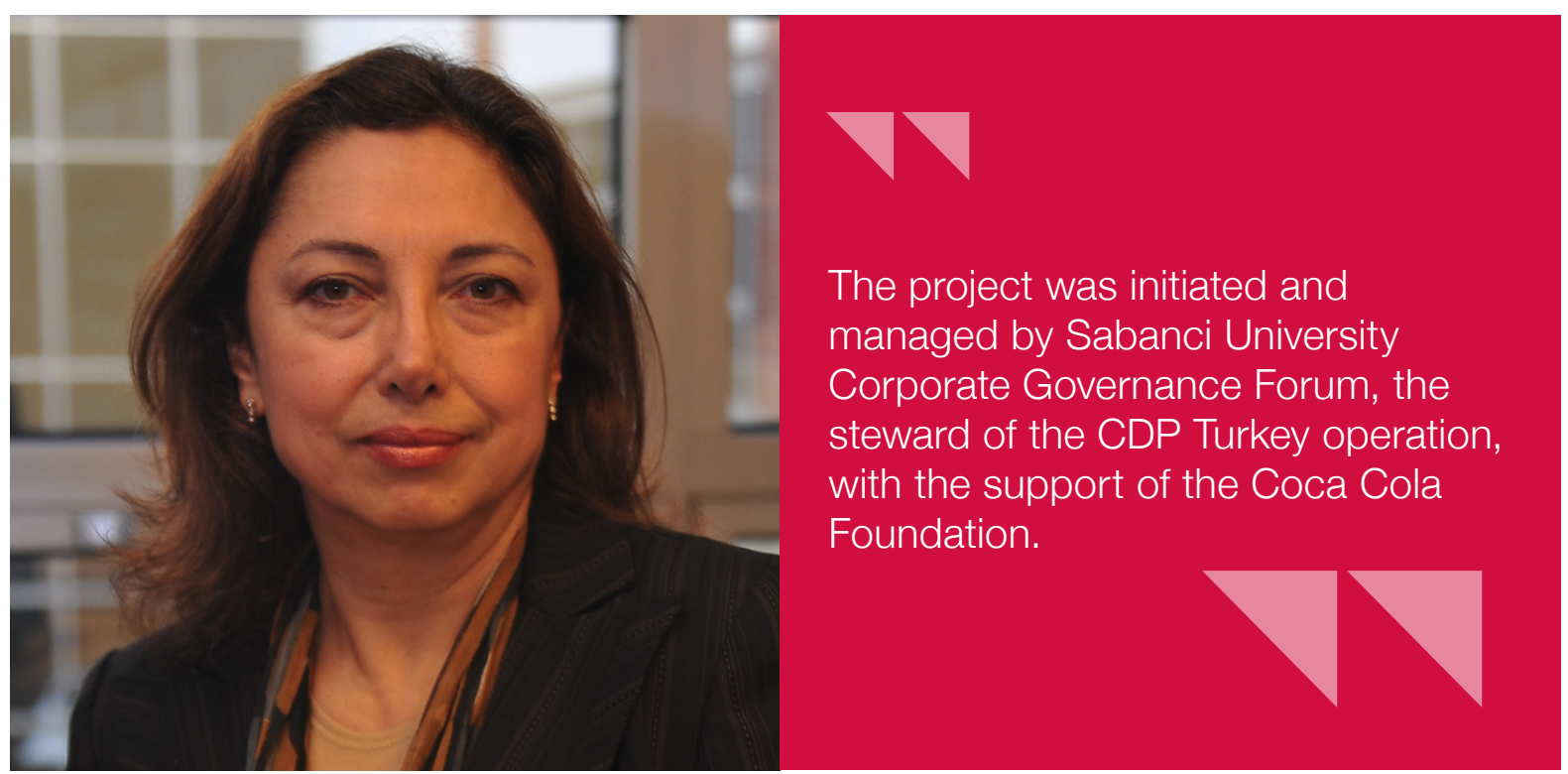

Environmental scientists have long been studying the effect of carbon emissions on the environment and the atmospheric climate. The implications of climate change for economic development and business organizations are expected to be profound. These changes are unprecedented and will transform the way we live and work. First of all climate change will affect the provision of energy and water and lead to restructuring of value chains, including supply networks. Second, organizational resilience and adaptability will be fundamentally more important than size. Third, the anticipated changes will demand different approaches to managerial behaviour and decision-making. All these underpin the concerns of long-term investors, the signatories of CDP, and their request for disclosure of climate change relevant information.

This report summarizes the outcome of our efforts to encourage Russian companies to disclose climate change information to investors and to the public through the CDP Russia 2014 project. The project was initiated and managed by Sabanci University Corporate Governance Forum, the steward of the CDP Turkey operation, with the support of the Coca Cola Foundation. The project aimed to transfer our knowledge and experience in mobilizing multiple stakeholders in Turkey to improve climate change disclosure and facilitate a discussion on how Russian companies can contribute to the global efforts in combatting climate change.

Sabanci University School of Management, who host the CDP Turkey operation, receives an increasing number of students from different countries every year, including from Russia. Our faculty tries hard to develop our students as internationally competent individuals, with a strong sense of social responsibility. Engaging with businesses through action projects like CDP in other countries helps our faculty to be better teachers and better scholars. Our attempt to share our experience in Turkey with Russian companies will undoubtedly add to our understanding of the interplay between regulatory interventions and market pressure in shaping business behaviour.

We hope that increasingly more Russian companies will use CDP as a platform to disclose information about their climate change risks, and improve their resilience in the disclosure process. We believe the information made available through their disclosures will also help the government to design better and more effective regulations that take into consideration the capacity and the needs of Russian businesses.

We are indebted to the Coca Cola Foundation for supporting this project, to EY Russia for their analysis and scoring of Russian companies as well as hosting workshops for companies, and to Michael Yulkin, our local advisor, without whom we would not be able to see this project through.

\section{Melsa Ararat, PhD}

Director, CDP Turkey

Sabanci University School of Management 


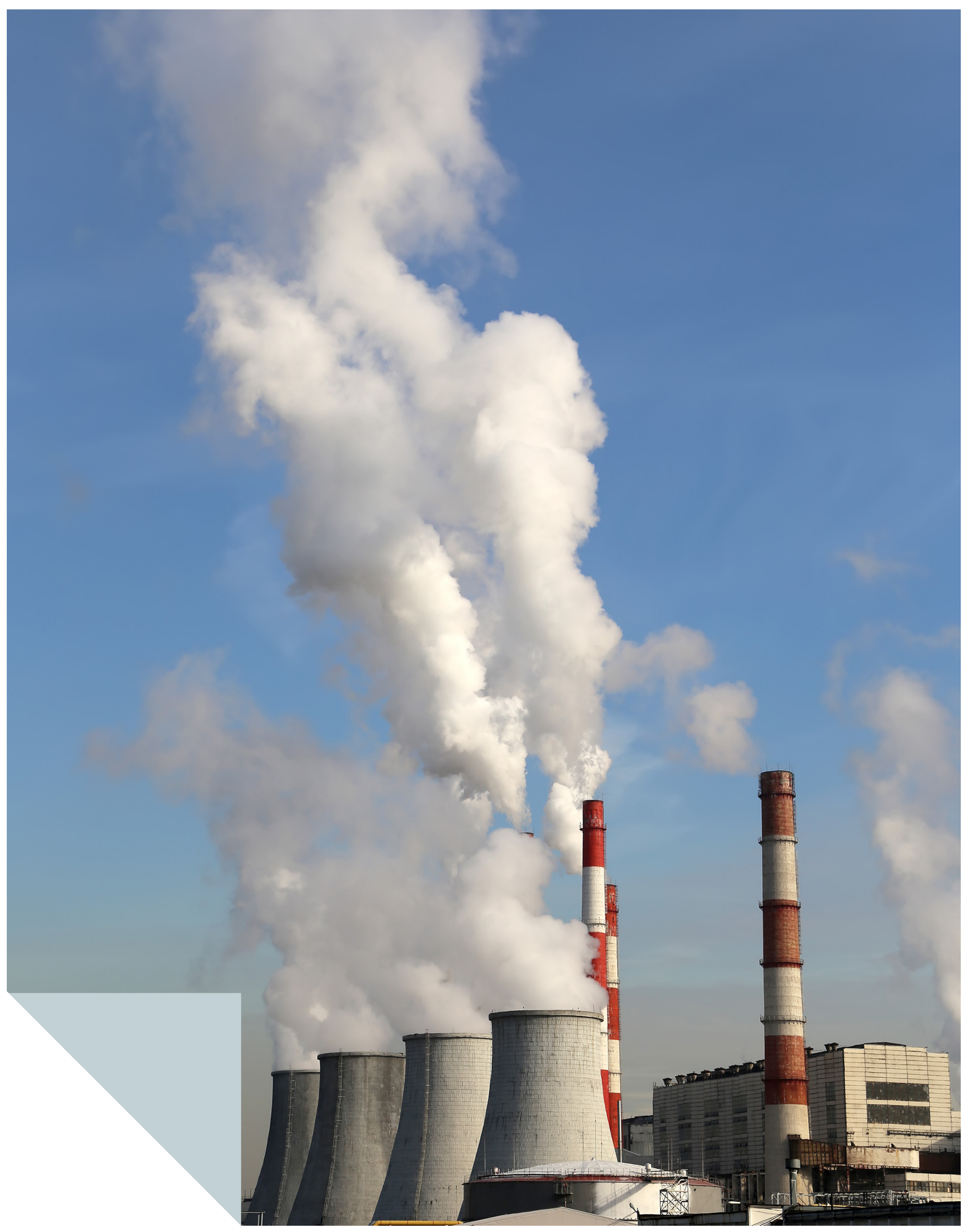




\section{EY Russia commentary}

EY Russia is proud to be the report writing and scoring partner for the CDP Russia Climate Change Report 2014. Our support of this project is just one of the many initiatives we, globally as a firm, are undertaking in order to help our clients transform into low carbon and energy efficient businesses, integrate their financial reporting with non-financial information and thus become part of the green economy.

As a resource-rich emerging economy strongly reliant on the production and sale of non-renewable hydrocarbons, Russia has seemingly little interest in making the transition to a low-carbon model. On the other hand, energy efficiency remains a clear priority of the Government and a prerequisite for global competitiveness. That is why energy efficiency improvements and greenhouse gas emission reduction are gaining importance for Russian enterprises, helping them manage risks and improve their market standing.

In 2013-2014 EY Russia completed research on industrial low carbon technology implementation in Russia as well as in Ukraine, Kazakhstan and Belarus that we titled "The Invisible Fuel". The survey demonstrated that the majority of participating companies recognize the importance of energy efficiency and are implementing effective technical and managerial solutions. Recent decisions from the President and the Government of the Russian Federation establishing national targets and an action plan on climate change, as well as wider publicity of the Russian meteorological agency's report on the negative effects of a changing climate should underscore the importance of business action against climate change.
In supporting this report we also hope that the positive experience of Russian participants of the CDP climate change program will help disseminate information on business response to climate change. This in turn will help wider business community recognize the need for specific action to reduce greenhouse gas emissions, adapt to the changing climate, and then to shift to a low-carbon growth model on macro and microeconomic levels.

Regrettably this year many largest Russian companies did not respond to the CDP information request. There may have been numerous reasons for that, but surely a lack of recognition of leaders in climate performance and transparency only contributed to the low response rate. The publication of the CDP Russia Climate Change Report 2014 and recognition from CDP of the best disclosure amongst Russian companies should help raise awareness about the challenges and opportunities that climate change and broader sustainability objectives are generating for businesses in Russia and globally.

We see that sustainability topics and in particular climate change issues are now more often being discussed in boardrooms and management meetings and taking their place among corporate strategy objectives. We would welcome further transparency and more action against climate change by our business leaders. Among other positive changes that would lead to increased participation and improved quality of responses to investors' requests for information via CDP Climate Change program next year.

Ksenia Leschinskaya, Partner, Head of Cleantech and Sustainability Services in the CIS 


\section{Russia's climate profile and key trends}

\section{Climate change: hitting the history highs}

In the Report on "Climate Features on the Territory of the Russian Federation in 2013"1 issued in 2014 Roshydromet, the Russian Federal Service for Hydrometeorology and Environmental Monitoring, confirmed that climate continues to warm across the country. From 1976 to 2013 the mean annual air temperature in Russia has been growing at an average rate of $0.43^{\circ} \mathrm{C}$ every 10 years - that is two times faster than the global average.

2013 was one of the hottest years on record for Russia since 1886. The mean annual temperature was $1.52^{\circ} \mathrm{C}$ above normal (1961-1990 average $)^{2}$ and $0.45^{\circ} \mathrm{C}$ above 2012 averages. The seasons in 2013 were characterized by a very warm autumn $\left(1.99{ }^{\circ} \mathrm{C}\right.$ above normal, the 3rd warmest since 1936) and summer (1.29 ${ }^{\circ} \mathrm{C}$ above normal, the 5th warmest since 1936). November 2013 was the warmest in the history of weather monitoring $\left(5.30^{\circ} \mathrm{C}\right.$ above normal).

2013 was also marked by extreme precipitation which, on average, appeared to be the highest since 1936. The spring rainfall marked the highest, and the autumn rainfall - the second highest on record. In winter 2012/13 the maximum depth of snow cover across Russia was $8.9 \mathrm{~cm}$ above normal on average.

According to Roshydromet, the largest contribution to the temperature rise across Russia in the late $20^{\text {th }}$ century to early $21^{\text {st }}$ century was from changes in greenhouse gas (GHG) concentrations in the atmosphere. $^{3}$ The data obtained at Roshydromet's monitoring stations confirm the upward trend in carbon dioxide concentration, which has increased by 21 million $^{-1}$, or by $5.7 \%$, over the last decade.

\section{GHG emissions: down from 1990, up from 2008}

As per the requirements of the United Nations Framework Convention on Climate Change (UNFCCC) and the Kyoto Protocol, Russia conducts an annual inventory of its anthropogenic GHG emissions from sources and removals by sinks and submits the National Inventory Report (NIR) as well as the National Communication on Climate Change to the UNFCCC Secretariat. NIR is verified by the independent international experts. In 2013, NIR covering the period from 1990 through 2011 was submitted and verified. ${ }^{4}$ According to the 2013 report, GHG emissions, excluding land use, land use change and forestry (LULUCF), grew in 2011 by 103.56 million metric tons CO2e marking a 4.47\% increase from 2010. In 2010 GHG emissions also increased by 4.52\% from 2009 levels, but that was mostly associated with the industry recovery following the drop caused by the economic crisis of 2009. Despite such growth, GHG emissions

1 http://www.meteorf.ru/upload/iblock/7ce/ob-osobennoctjach-klimataRF-2013.pdf

2 Mean annual global surface temperature in 2013 was $0.50^{\circ} \mathrm{C}$ above normal. 3 See Second Assessment Report on Climate Change and Its Consequences in the Russian Federation, 2014 - http://voeikovmgo.ru/download/2014/od/ od2.pdf

4 Go to http://unfccc.int/national_reports/annex_i_ghg_inventories/national inventories_submissions/items/7383.php
Figure 1 Russia's overall GHG emissions in 2008-2011 (excluding LULUCF), million metric tons $\mathrm{CO}_{2} \mathrm{e}$

2350

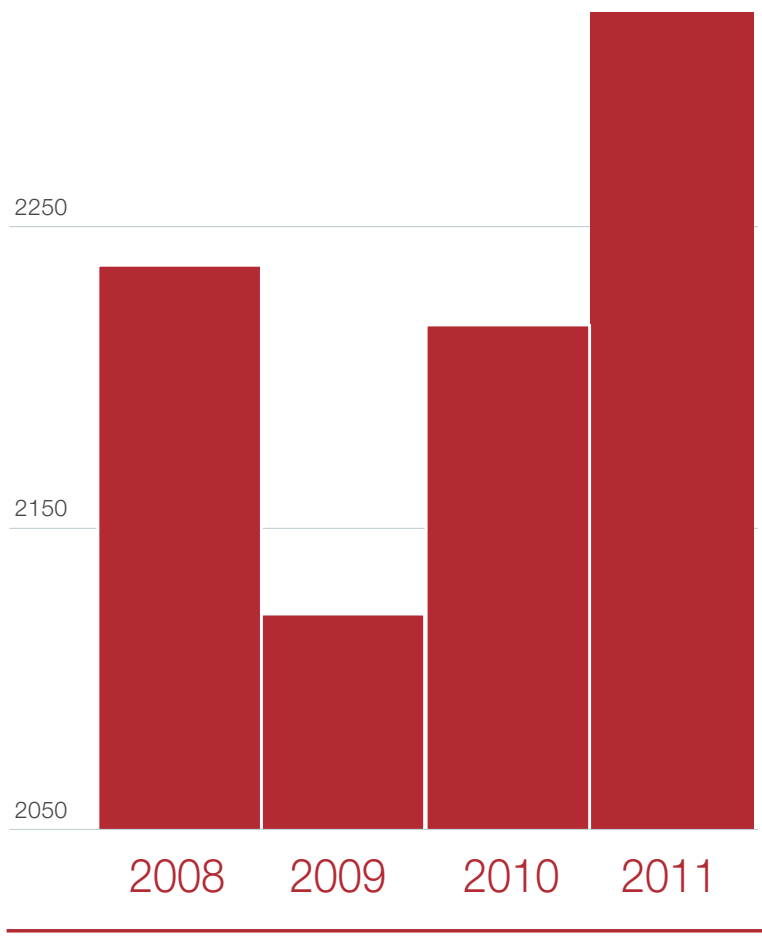

Source: 2013 Russian Federation GHG Inventory Submission to UNFCCC (http://unfccc.int/national_reports/annex_i_ghg_inventories/national_inventories_ submissions/items/7383.php)

in 2010 remained below pre-recession levels of 2008. However, in 2011, GHG emissions were 83.41 million metric tons $\mathrm{CO}_{2} \mathrm{e}$, or $3.73 \%$, higher than 2008 levels. $^{5}$

A more detailed breakdown of GHG emissions in 20082011 is provided in Table 1.

5 In 2014, Russia submitted new NIR (2014 NIR) covering the period 19902012 (available on the UNFCCC website http://unfccc.int/national_reports/ annex_i_ghg_inventories/national_inventories_submissions/items/8108.php) in which new estimates of GHG emissions over 1990-2011 period are provided alongside GHG emission estimates for 2012. According to this 2014 NIR, GHG emissions (excluding LULUCF) in Russia went up by $1.71 \%$ in 2011 and by emissions (excluding LULUCF) in Russia went up by $1.71 \%$ in 2011 and by
$2.2 \%$ in 2012 compared to 2008 level. Without any prejudice to these numbers that might very well be true, it has to be noted here that GHG emissions reported in 2014 NIR appeared to be a bit higher than in the previous 2013 NIR for each and every year from 1990 through 2010 and lower than that - for the year 2011. Particularly, the new GHG estimate for 1990 base year is 11.4 million metric tons $\mathrm{CO}_{2} \mathrm{e}$ and for $2008-8.4$ million metric tons $\mathrm{CO}_{2} \mathrm{e}$ up from $2013 \mathrm{NIR}$, while the newly reported emissions in 2011 are 35.6 million metric tons $\mathrm{CO}_{2} \mathrm{e}$ down from 2013 NIR. The reason for that is not clear. However one should bear down from 2013 NIR. The reason for that is not clear. However one should bear
in mind that 2014 NIR has not been verified yet. That's why further on in this report only verified data provided in 2013 NIR are considered. 
The CCGS study ${ }^{1}$ shows that GHG emissions growth between 2008 and 2011 was mainly caused by:

- Motor vehicles: $\mathrm{CO}_{2}$ emissions from fuel combustion increased by 64.7 million metric tons $\mathrm{CO}_{2} \mathrm{e}$;

- Oil and gas industry, including oil refining: GHG emissions grew up by 30.6 million metric tons $\mathrm{CO}_{2} \mathrm{e}$ due to:

- methane leakages from oil and gas production, transportation and storage - 10.7 million metric tons $\mathrm{CO}_{2} \mathrm{e}$,

- natural gas and associated petroleum gas flaring 10.6 million metric tons $\mathrm{CO}_{2} \mathrm{e}$,

- fuel combustion for oil refining - 9.2 million metric tons $\mathrm{CO}_{2} \mathrm{e}$;

- Other industries: $\mathrm{CO}_{2}$ emissions from fuel combustion grew by 10.2 million metric tons $\mathrm{CO}_{2} \mathrm{e}$;

- Housing and public utilities: $\mathrm{CO}_{2}$ emissions from fuel combustion grew by 7.8 million metric tons $\mathrm{CO}_{2} \mathrm{e}$;

- Waste management: methane emissions grew by 6.5 million metric tons $\mathrm{CO}_{2} \mathrm{e}$.
Over the same period, the largest reductions in GHG emissions were due to:

- Coal industry: GHG emissions reduced by 12.2 million metric tons $\mathrm{CO}_{2} \mathrm{e}$;

- Agriculture, fishing industry and forestry: overall GHG emission reduced by 9.6 million metric tons $\mathrm{CO}_{2} \mathrm{e}$;

- Chemical industry: GHG emissions from industrial processes reduced by 7.4 million metric tons $\mathrm{CO}_{2} \mathrm{e}$;

- Iron and steel industry: $\mathrm{CO}_{2}$ emissions from fuel combustion reduced by 5.8 million metric tons $\mathrm{CO}_{2} \mathrm{e}$;

- Transport (other than motor vehicles): $\mathrm{CO}_{2}$ emissions from fuel combustion reduced by 5.6 million metric tons $\mathrm{CO}_{2} \mathrm{e}$.

In 2011, Russia's GHG emissions stood 30.8\% below their base year (1990 level) without LULUCF $(50.8 \%$ if LULUCF is considered)2. However, emissions have increased from 1990 base levels by $15.3 \%$ in the oil and gas industry, by $5.1 \%$ in the motor transport, and by $31.1 \%$ in the housing and public utilities (see Table 2).

Table 1 Russia's GHG emissions in 2008-2011 (excluding LULUGF), million metric tons $\mathbf{C O}_{2} \mathrm{e}$

\begin{tabular}{|c|c|c|c|c|}
\hline GHG emissions & 2008 & 2009 & 2010 & 2011 \\
\hline Carbon dioxide $\left(\mathrm{CO}_{2}\right)$ - total & 1609.3 & 1526.4 & 1598.2 & 1684.4 \\
\hline \multicolumn{5}{|l|}{ Including: } \\
\hline - emissions due to fuel combustion & 1426.8 & 1360.6 & 1411.7 & 1491.1 \\
\hline - emissions due to industrial processes & 157.2 & 139.7 & 153.3 & 157.3 \\
\hline Methane $\left(\mathrm{CH}_{4}\right)$ - total & 492.9 & 464.7 & 491.1 & 506.6 \\
\hline \multicolumn{5}{|l|}{ Including: } \\
\hline $\begin{array}{l}\text { - leakage from production, storage and } \\
\text { transportation of fuel, including flaring of } \\
\text { natural and associated petroleum gases }\end{array}$ & 372.7 & 341.2 & 369.2 & 382.1 \\
\hline - emissions due to waste handling & 70.3 & 74.1 & 73.6 & 76.6 \\
\hline Nitrogen oxide $\left(\mathrm{NO}_{2}\right)$ & 116.2 & 116.8 & 113.8 & 117.6 \\
\hline PFCs & 3.7 & 2.5 & 2.7 & 2.5 \\
\hline HFCs & 14.4 & 10.1 & 10.9 & 9.1 \\
\hline SF6 & 0.8 & 0.8 & 0.7 & 0.5 \\
\hline Total GHG emissions & 2237.4 & 2121.4 & 2217.3 & 2320.8 \\
\hline
\end{tabular}

Source: 2013 Russian Federation GHG Inventory Submission to UNFCCC (http://unfccc.int/national_reports/annex_i_ghg_inventories/national_inventories_submissions/ items/7383.php)

$1 \mathrm{M}$. Yulkin, D. Schekoldin. Analysis of a sectoral structure and dynamics of the GHG emissions in Russia in 2011. http://ccgs.ru//publications/articles/ download/analysis.GHG
2 According to 2014 NIR, in 2011 GHG emissions in Russia reduced from 1990 level by $32.1 \%$ without LULUCF and by $51.5 \%$ with LULUCF included; in 2012 by $31.8 \%$ and $50.3 \%$, respectively. 
Table 2 GHG emissions by sectors in 1990 and 2011 (excluding LULUCF),

million metric tons $\mathrm{CO}_{2} \mathrm{e}$

\begin{tabular}{|c|c|c|c|}
\hline Sectors & 1990 & 2011 & $\begin{array}{r}\text { Emissions in } 2011 \text { as } \\
\text { a percentage of } 1990 \\
\text { emissions }\end{array}$ \\
\hline GHG emissions - total & 3351.9 & 2320.8 & 69.2 \\
\hline \multicolumn{4}{|l|}{ Of the total amount: } \\
\hline Fuel and energy complex & 1608.5 & 1317.4 & 81.9 \\
\hline \multicolumn{4}{|l|}{ Including: } \\
\hline - Power generation & 1176.0 & 829.6 & 70.5 \\
\hline - Oil and gas, including crude oil refining & 359.4 & 414.4 & 115.3 \\
\hline $\begin{array}{l}\text { - Coal industry and other industries of Fuel and } \\
\text { Energy Complex }\end{array}$ & 73.0 & 73.4 & 100.6 \\
\hline Production of goods and construction & 475.0 & 320.9 & 67.6 \\
\hline \multicolumn{4}{|l|}{ Including: } \\
\hline - Iron and steel industry and non-ferrous industry & 203.7 & 143.0 & 70.2 \\
\hline $\begin{array}{l}\text { - Chemical industry, including manufacturing of } \\
\text { polymers }\end{array}$ & 51.0 & 35.6 & 69.7 \\
\hline $\begin{array}{l}\text { - Other industries of Production and Construction } \\
\text { sector }\end{array}$ & 220.2 & 142.3 & 64.7 \\
\hline Agriculture, forestry and fishing industry & 379.1 & 165.1 & 43.5 \\
\hline Transport - total & 342.4 & 283.6 & 82.8 \\
\hline \multicolumn{4}{|l|}{ Including: } \\
\hline - Motor transport & 177.5 & 186.7 & 105.1 \\
\hline - Pipeline transport & 110.5 & 80.2 & 72.6 \\
\hline - Other types of transport & 54.3 & 16.7 & 30.8 \\
\hline Housing and public utilities & 92.7 & 121.6 & 131.1 \\
\hline Other industries & 393.2 & 31.5 & 8.0 \\
\hline Waste management & 61.1 & 80.9 & 132.3 \\
\hline
\end{tabular}

Source: CCGS, http://ccgs.ru//publications/articles/_download/analysis.GHG 


\section{Mitigation and adaptation: targeting measures and measuring targets}

The Russian Government's activities in climate change mitigation and adaptation build upon the "Climate Doctrine of the Russian Federation"1 and the "Comprehensive Plan for Implementation of the Climate Doctrine for the Period up to 2020". 2 The first "Progress Report for the Climate Doctrine Implementation Plan" was published by the Russian Ministry of Natural Resources and Environment in November 2013; 3 the second report summarizing the results of 2013 was released in April 2014. ${ }^{4}$

According to the report, the Russian Government implemented various measures in 2013 to facilitate climate change mitigation and to ensure GHG emissions reductions in different sectors. Those measures included:

- Development and implementation of cross-sectoral GHG limitation strategies, including:

- Economic instruments for GHG emissions limitation and reduction from the industry;

- Reducing market distortions, improving financial and tax policies to provide incentives for GHG emission limitation and reductions;

- GHG emissions limitation in industry and energy sectors, including:

- Measures aiming to increase the use of renewable energy sources;

- Introduction of innovative nuclear energy technologies;

- Development and implementation of innovative utilization methods for methane containing emissions;

- Development and implementation of GHG emissions limitation in transport sector, including:

- GHG emissions limitation measures in civil aviation;

- Enhancing energy efficiency of Russian marine ships and river transport vessels;

- GHG emissions limitation in design and operation of buildings and structures, including:

- Measures to extend services provided by energy service companies;

- Implementation of energy metering systems in buildings;

- Operations improvement in forestry and agricultural sectors, including:

- Protection and quality improvement of forests that serve as natural carbon sinks and reservoirs,

1 Adopted by the Order of the Russian President No. 861-rp of December 17, 2009 (http://kremlin.ru/acts/6365)

2 Adopted by the Resolution of the Russian Government No. 730-r of April 25 2011 (http://www.rg.ru/2011/05/03/klimat-doktrina-site-dok.html)

3 http://www.mnr.gov.ru/regulatory/detail.php?ID=131748

4 http://www.mnr.gov.ru/regulatory/detail.php?ID=134236 application of sustainable forest management methods;

- Providing incentives for climate change adaptation activities in agriculture.

The most important event of the year was the Russian President's "Decree on Greenhouse Gas Emissions Reduction" (No. 752 of September 30, 2013) ${ }^{5}$ which sets a short-term goal to reduce GHG emissions by 2020 by at least $25 \%$ below 1990 levels. ${ }^{6}$ Pursuant to this Decree, the Russian Government developed and, in April 2014, approved the Action Plan to achieve this goal. ${ }^{7}$ The plan consists of three parts:

- Implementation of GHG emissions accounting and reporting systems at both regional and corporate level;

- Projection of GHG emissions for the period up to 2020 and towards 2030 as well as GHG emissions reduction potential by economy sectors;

- Development of GHG emissions regulation mechanism, including the mechanism aimed at providing incentives and support for GHG emissions reduction projects.

Much attention was also given to short-term and longterm climate change adaptation measures aimed at reducing natural disaster damage and other negative climate change effects by taking preventive actions and building capacities to effectively respond to negative climate events. "The 2014 Progress Report on the Execution of the Climate Doctrine Implementation Plan" states that measures and solutions were developed in 2013 to address issues such as:

- Minimization of disease and death rates among high risk population groups, including the spread of infection and parasitic diseases caused by climate change;

- Development and implementation of measures aiming to prevent growth in the frequency of forest and peat fires and to minimize their negative effects in the context of a higher risk of drought in some regions of the Russian Federation;

- Mitigation of production loss risks in agriculture (including the risk of reduction in livestock productivity and crop yields);

- Limiting negative impacts caused by more frequent floods due to higher rainfall, flood rate and rising sea levels;

- Mitigation of mountain glaciation degradation, dangerous mudflow and avalanche activities;

- Development and implementation of a set of measures aiming to reduce the impacts of hurricanes, including damage caused to power lines and facilities.

5 See http://www.kremlin.ru/acts/19344

6 The Decree does not explicitly clarify whether LULUCF net impact is considered or not. However the common understanding tends to exclude LULUCF which actually generates net GHG removals rather than GHG emissions in Russia. Still the uncertainty remains and should be accounted for. 7 See http://government.ru/media/files/41d4d0082f8b65aa993d.pdf 
In 2013, several Russian regions set about developing regional climate strategies. The first regional strategy was developed as early as 2009 for the Murmansk Region. In mid-2013 the Government of St Petersburg announced drafting of a climate change adaptation strategy.

Finally, at the end of 2013 the Arkhangelsk Regional Government initiated the development of a climate strategy for the Russian sector of the Barents EuroArctic Region. This region includes five constituents of the Russian Federation, namely: Murmansk and Arkhangelsk Regions, Republic of Karelia, Komi Republic and Nenets Autonomous Area. It is expected that the project will be implemented within the framework of Barents cooperation in accordance with the "Climate Change Action Plan" adopted at the $11^{\text {th }}$ Meeting of the Environment Ministers of the Barents Euro-Arctic Council (BEAC) held in Inari (Finland) on December 5, 2013.

Despite the measures undertaken at the federal and regional levels, GHG emissions in Russia have continued to grow, the carbon intensity of the Russian economy (GHG emissions per \$1 PPP GDP) remains one of the world's highest ${ }^{2}$ affecting the competitiveness of domestic producers that are facing increasing pressure from clients interested in reducing their carbon footprint. Hydro-meteorological (weather-linked) disasters are becoming more abrupt and inflict higher damages. ${ }^{3}$

\section{Involving Russian business in climate actions}

Prior to 2013, the participation of Russian businesses in climate change mitigation actions was limited mainly to Joint Implementation (JI) projects under Article 6 of the Kyoto Protocol. Some companies took further steps and made long-term commitments to climate change mitigation and adaptation as an integral part of their business strategy aimed at increasing their value and strengthening their position in the market. However for the majority of Russian companies Jl providing the opportunity to sell emission reductions achieved in the course of projects implementation was the only economic reason to involve in climate change actions.

$1 \mathrm{http} / / / \mathrm{www}$. barentsinfo.fi/beac/docs/Environment_Ministers_Meeting_4_5_ Nov_2013_Inari_Action_Plan_Climate_Change_ENG.pdf

2 According to IEA, in 2012 Russia's $\mathrm{CO}_{2}$ emissions from fuel combustion per $\$ 1$ PPP GDP amounted $0.76 \mathrm{~kg} \mathrm{CO}_{2} / \mathrm{US}$ dollar while the world's average was $0.38 \mathrm{~kg} \mathrm{CO}_{2} / \mathrm{US}$ dollar and OECD average $-0.31 \mathrm{~kg} \mathrm{CO}_{2} / \mathrm{US}$ dollar (please see http://www.iea.org/publications/freepublications/publication/KeyWorld2014. pdf).

pdf).
3 In September 2013 Russia's Far East was badly hit by severe floods caused 3 In September 2013 Russia's Far East was badly hit by severe floods caused
by heavy rains and catastrophic rising of the water levels in the Amur River. For a few days the flow in the Amur stayed at 2.5-3 times its normal rate. No such floods have ever been registered in the region over the 115-year monitoring period. Due to the disaster, over 200 population centers, 11,000 residential houses with over 70,000 residents, and around 50,000 private households and hom with to the agricultural million). A year earlier catastrophic floods caused by heavy rains hit Krasnodar Krai in the Russian South. Torrential rains led to the Rivers Aderba, Bakanka and Adagum bursting their banks and battering the town of Krymsk and other settlements like a tsunami. The water level of the flow was as high as 4 and sometimes even 7 meters. The flooding affected over 34,000 people, claimed 171 lives, destroyed or damaged 7,200 residential houses. It also affected gas, energy and water supply infrastructure. The economic costs of the floods are energy and water supply infrastructure. The economic costs of the floods
estimated at about RUR 20 billion (USD 600 million). The insurance claims estimated at about RUR 20 billion (US
totaled RUR 1 billion (USD 35 million).
Russia's domestic regulation for Jl projects approval and implementation was adopted in 2007, but then it changed two times, in 2009 and 2011. Finally, Sberbank of Russia was appointed as the authorized Carbon Units Operator to facilitate $\mathrm{Jl}$ projects evaluation and selection as well as Emissions Reduction Units (ERUs) issuance and transfer.

The first Jl project was approved by the Russian Ministry of Economic Development in June 2010, the last one - in May 2012. Over this period $108 \mathrm{Jl}$ projects submitted by 75 companies were approved. Altogether these projects were estimated to result in 311.5 million metric tons $\mathrm{CO} 2 \mathrm{e}$ of $\mathrm{GHG}$ emission reductions between 2008 and 2012. By the end of 2013 in total 263.4 million ERUs ${ }^{4}$ were issued for $86 \mathrm{Jl}$ projects. Of this amount, 237.5 million ERUs from 74 projects were sold and transferred to foreign buyers. ${ }^{5}$

At present joint implementation is no longer an opportunity for Russian business since Russia has refused to assume any quantitative commitments with regard to GHG emissions limitation and reductions in the second Kyoto Period (2013-2020) which makes the Kyoto flexible mechanism unavailable. Another important factor was the slump in the EU carbon market which was the only available market for Russian ERUs. ERU prices in Europe dropped down to several cents per unit, which caused disappointment among Russian sellers.

Still Russian companies remain committed to GHG emission limitation and reduction. More so, the year 2013 saw an example of Russian business moving from implementation of isolated emission reduction projects to regular GHG emissions monitoring, accounting and management as per international standards.

Arkhangelsk Pulp and Paper Mill (Arkhangelsk PPM) took the lead as the first Russian company to certify its corporate GHG management systems to the ISO 14064-1:2006 standard. In addition to monitoring, accounting, and reporting, Arkhangelsk PPM's GHG emissions management system also includes an emission limitation and reduction strategy for the period up to 2020 and an action plan to ensure that the targets set forth in this strategy are achieved.

The Russian Government is now planning to make carbon reporting mandatory for major Russian companies. The decision to introduce it has already been made and the details of its implementation are included in the Russian Government's Action Plan approved in April 2014. It is also anticipated in the Action Plan that, by the end of the current decade, GHG emissions control and regulation mechanisms will be introduced including the mechanisms to incentivize and support GHG emission reduction projects.

$41 \mathrm{ERU}=1$ metric ton $\mathrm{CO}_{2} \mathrm{e}$.

5 In fact the numbers could have been even more impressive. But in 2011 the Russian Government decided to put a 300 million metric tons $\mathrm{CO}_{2}$ e limit on $\mathrm{Jl}$ projects by capping the overall number of ERUs that can be issued for the projects. Later this limit was abandoned, however the overall carbon value of approved JI projects still remained capped. 
Figure 2 Issue and transfer of ERUs under Russian Jl projects (as of 27.11.2013), million units

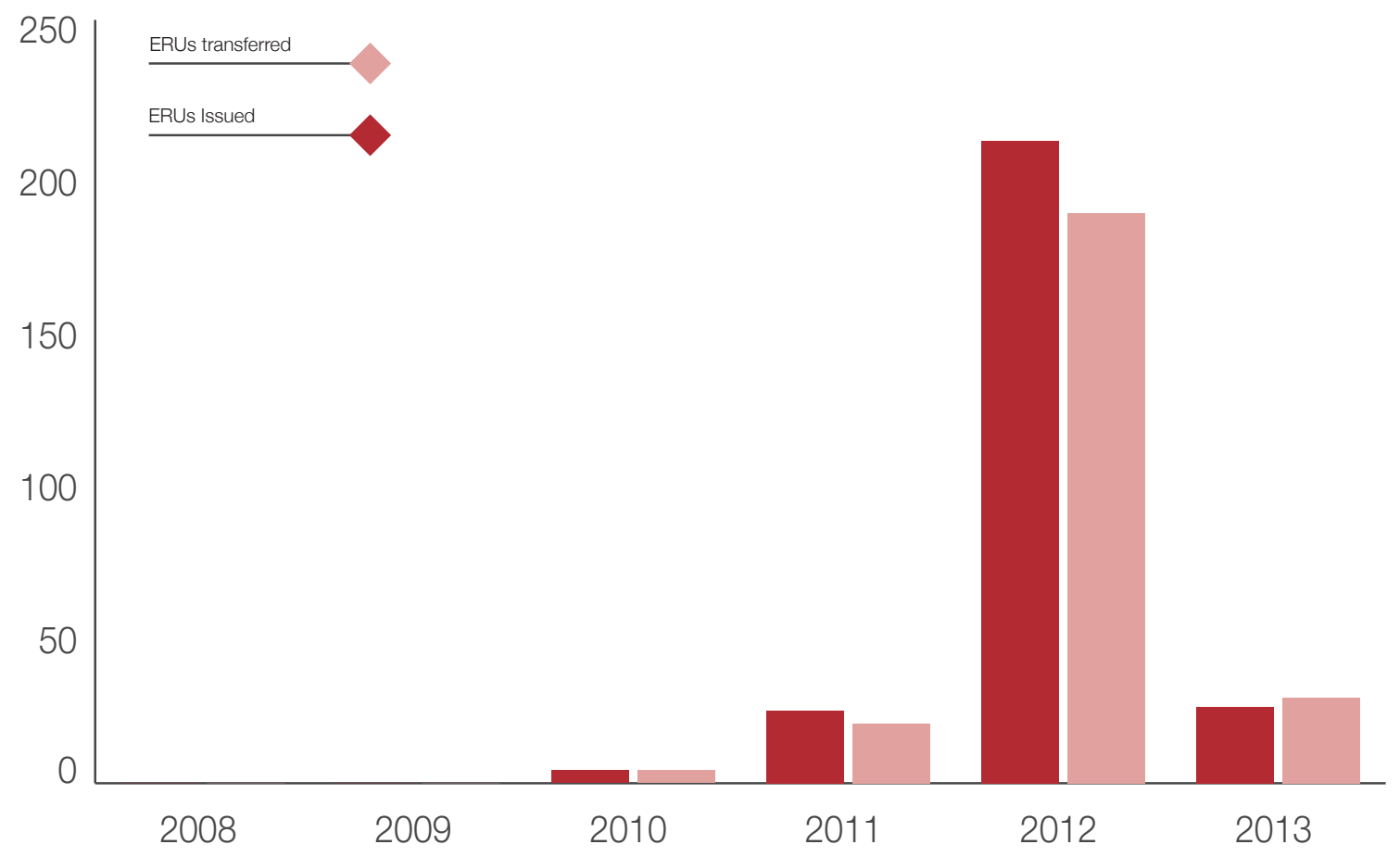

Source: calculated using the data from the official Russian Carbon Registry's website (www.carbonunitsregistry.ru)

A few platforms are used for involving businesses in the discussion of GHG emissions monitoring, reporting and regulation. The most representative is the Joint Working Group on GHG Emissions Regulation, which was established on the initiative of the Russian Ministry of Economic Development and Delovaya Rossiya business union with participation of the Russian Union of Industrialists and Entrepreneurs (RSPP) and some other business associations. The Interagency Working Group on climate change and sustainable development under the Administration of the Russian President also includes representatives of RSPP and Delovaya Rossiya. $^{1}$

\section{Climate change and social activism}

Climate change mitigation and adaptation are high on the agenda of Russian non-governmental organizations (NGOs). The efforts of WWF Russia ${ }^{2}$ in Moscow and of the Socio-Ecological Union ${ }^{3}$ in St Petersburg should be recognized. WWF Russia has a dedicated Climate and Energy Program, and the Socio-Ecological Union has set a Climate Secretariat specifically to deal with climate change issues. Among NGOs that were set up recently to focus specifically on climate change, $350 . \mathrm{org}^{4}$, the Russian arm of the same international environmental organization, should be mentioned as a good example.

1 The working group was set up in 2013 following the order of the Russian President No. 563-rp of December 13, 2012.See http://graph.document. kremlin.ru/page.aspx?1642603

2 http://www.wwf.ru

$3 \mathrm{http}: / /$ www.rusecounion.ru

4 http://www.world.350.org/russian
Russian NGOs employ highly qualified staff with strong experience in energy and climate issues, conduct large-scale awareness-raising campaigns, prepare and publish research, reviews and articles on their websites, organize meetings and conferences, come forth with various initiatives, and participate as observers in international climate negotiations. They also sit in all major climate change working groups in Russia and make invaluable contributions towards understanding of the current situation and trends and elaboration of appropriate solutions to climate-related challenges.

\section{Looking forward and beyond}

Having come to a consensus on its short-term plans towards 2020, Russia is still hesitant about its longerterm climate and GHG emission reduction strategy. Scientific groups, government bodies, NGOs, and business associations put forward forecasts and visions on what this strategy should look like and what opportunities Russia has to contribute to global climate change mitigation efforts by reducing its $\mathrm{GHG}$ emissions. Some of these studies demonstrate that Russia's GHG emissions can stabilize after 2020 and even reduce by half (compared to 1990) if certain policies and measures are undertaken.

The "Forecast of Socio-economic Development of the Russian Federation by 2030"5 adopted by the Russian Government on March 26, 2013 suggests that Russian GHG emissions will reach $75 \%$ of 1990 levels by 2020 ,

$\overline{5 \text { See www.economy.gov.ru }}$ 
Figure 3 Scenarios of CO2 emissions from fuel combustion in Russia towards 2050, million metric tons $\mathrm{CO}_{2} \mathrm{e}$

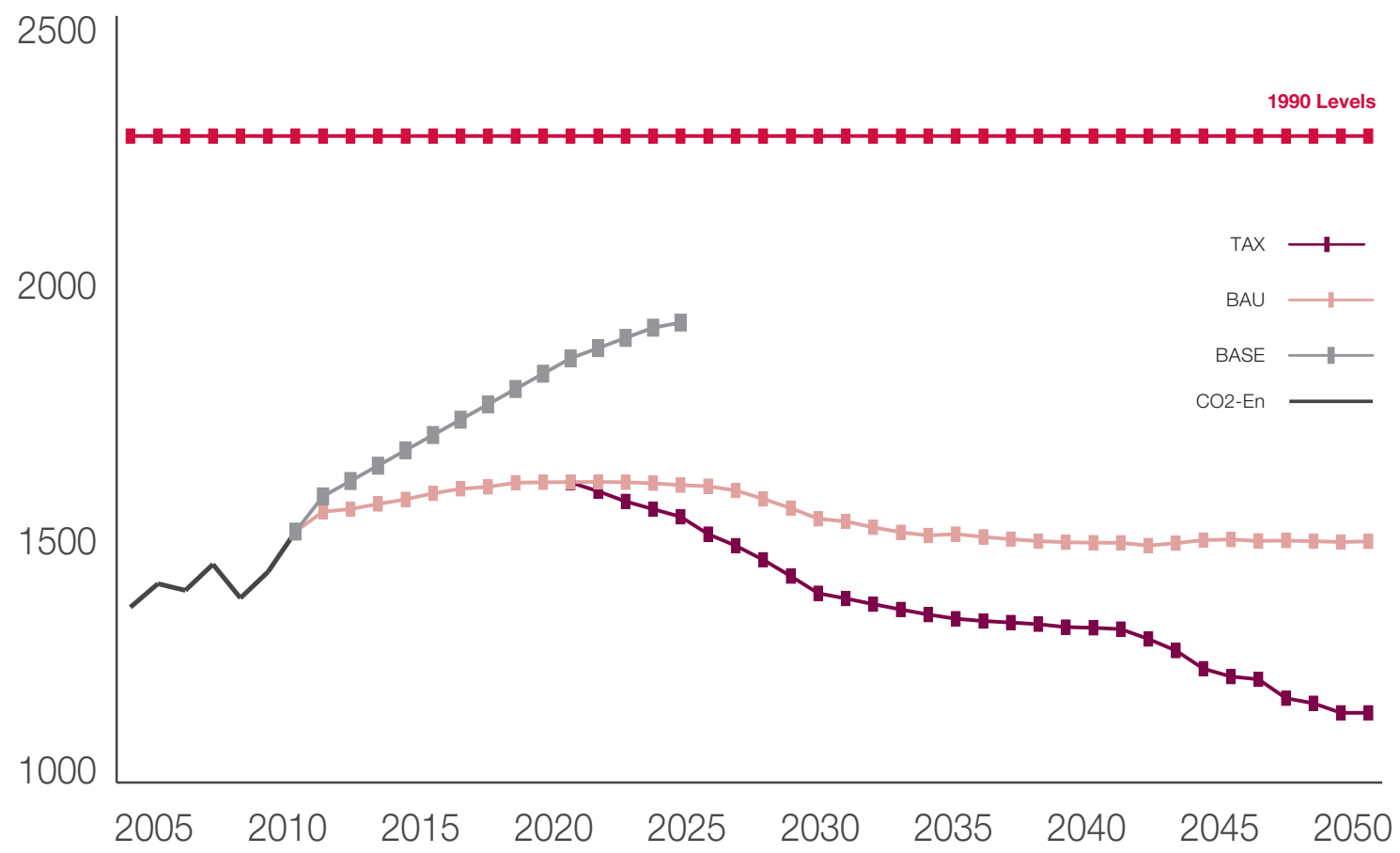

CO2-En - CO2 emissions from fuel combustion in 2005-2011; BASE - Hypothetical scenario, only old technologies deployed; BAU - Business-as-usual scenario at 3\% GDP growth rate; TAX - Carbon tax scenario aimed at reducing GHG emissions by 50\% of 1990 level in 2050 (carbon tax grow from 15 USD/t CO2 in 2020 to 50 USD/t CO2 in 2050 ) Source: A. O. Kokorin, I.G. Gritsevich \& D.S. Gordeev Greenhouse Gas Emission Scenarios for Russia and Rest of the World, 2013, Review of Business and Economic Studies (ROBES), vol.1, no. 1, pp.55-66. http://www.fa.ru/projects/rbes/about/Pages/default.aspx

thereafter continue to decline reaching $70 \%$ in 2030 as a result of energy-efficiency measures, increased labour productivity, and renewable energy policies. ${ }^{1}$

The recently renewed "State Program on Energy Efficiency and Energy Development (for the period till 2035)"2 presented by the Ministry of Energy suggests that a $40 \%$ reduction in GDP energy intensity by 2020 will be achieved partly through technological improvements (13.5\%) and most importantly through structural transformation of the economy (26.5\%). With this trend, $\mathrm{CO}_{2}$ emissions from the energy sector (contributing $80 \%$ of Russia's overall GHG emissions) will grow to reach $79.3 \%$ of 1990 level by 2035 after achieving about $75 \%$ of the 1990 levels in 2020 .

$\mathrm{BP}$ has come to the same figures in their "Energy Outlook 2035". ${ }^{3}$ According to BP, $\mathrm{CO}_{2}$ emissions from the energy sector in Russia will rise as high as $80 \%$ of 1990 levels by 2035, with the forecast for 2020 at $73-75 \%$. The Energy Institute of the Russian Academy of Science (ERI RAS) predicts in its turn that $\mathrm{CO}_{2}$ emissions from fuel combustion for energy generation will stabilize in the 2030s at $85-88 \%$ of 1990 levels after reaching $81-83 \%$ in $2020 .^{4}$

1 LULUCF net impact is not considered. This also applies to all other studies mentioned in this section.

2 Ministry of Energy. Alexey Kulapin. Presentation of the New Version of the State Program on Energy Efficiency and Energy Development (for the period by 2036). Analytical Centre of the Russian Government, Moscow, 27 Jan. 2014. 3 BP-2035, C. Ruhl, Energy Outlook 2035, IMEMO, Moscow, 17 Jan. 2014 www.bp.com/energyoutlook

4 ERI RAS, Alexey Makarov, Scenarios of Russian Energy Strategy, Gaidar
The Centre for Energy Efficiency (CENEf) has estimated $\mathrm{CO}_{2}$ emissions reductions under different policy scenarios. ${ }^{5}$ According to their estimates, the Current Policy scenario will lead to $70 \%$ of 1990 level by 2020 given a conservative $2 \%$ GDP growth rate; and to $75 \%$ if a $3 \%$ GDP growth rate is considered. However, in the outlook for the 2030s, the Current Policy scenario shows $\mathrm{CO}_{2}$ emissions stabilizing at $80 \%$ of 1990 levels assuming GDP growth at 3\% on average.

The New Policy scenario assumes implementation of measures already announced by the Russian Government as desirable including the wide use of energy service companies, certification, subsidies, sufficient growth of labour productivity, fuel saving in motor vehicles, etc. With this policy GHG emissions may reach $70-74 \%$ of 1990 levels by 2020 and stabilize thereafter at this level.

Finally, the so called Resolute Policy scenario entails wide support to renewable energy, biofuels and nuclear energy, sufficient carbon price and implementation of Carbon Capture and Storage (CCS) technology if the carbon price exceeds $58 \mathrm{USD} / \mathrm{t} \mathrm{CO} \mathrm{C}_{2}$. These measures could reduce $\mathrm{CO}_{2}$ emissions down to $60-65 \%$ of the 1990 levels by 2020 and further to $50 \%$ by 2050 .

Economic Forum, Moscow, 16 Jan. 2014

5 CENEf, I. Bashmakov, Scenarios of Russian Socio-Economic Development by Low-Carbon Trajectories, Gaidar Economic Forum, Moscow, 16 Jan. 2014. 
The Institute of Economic Policy (IEP) and the Russian

Presidential Academy of the National Economy and Public Administration (RANEPA) jointly estimated Russia's future GHG emissions for a number of scenarios using a well-known TIMES model. ${ }^{1}$ The calculations show that the business-as-usual (BAU) scenario will see minor GHG emissions growth until 2015 and stabilization by 2020 at $72-75 \%$ of the 1990 levels while this level persists in the next decades. In case carbon tax is introduced starting from $15 \mathrm{USD} / \mathrm{t} \mathrm{CO}$ in 2015 and gradually increasing to $50 \mathrm{USD} / \mathrm{t} \mathrm{CO}_{2}$ by 2050, GHG emissions might decrease to $60-65 \%$ of the 1990 levels by the end of the period. IEP and RANEPA have also prepared the scenarios, CAP50 and CAP75, that demonstrate the feasibility of limiting GHG emissions to $50 \%$ and ambitious 25\% of the 1990 level by 2050; both include enhanced biofuel use and CCS.

Some experts, however, do not agree that from now on Russia's economy can only grow slowly as it is assumed in the above studies, and challenge their GHG forecasts by applying higher growth rates. The IEA analysed the same policy scenarios as CENEf and IEP-RANEPA based on higher economic growth rate assumptions and come to the following conclusions:

With the Current Policy scenario GHG emissions will reach $80 \%$ of the 1990 level by 2020 and $90 \%$ by 2030;

With the improved New Policy scenario emissions will not exceed $78 \%$ of 1990 level in 2020 and will then grow to $82.5 \%$ of 1990 level by 2035;

Only targeted 450 ppm scenario shows stabilization of $\mathrm{CO}_{2}$ emissions at $73.5 \%$ of the 1990 level in 2020 and then decarbonisation to $58 \%$ of the 1990 level by 2030 and to $52 \%$ by 2035.

The final decisions about Russia's GHG emissions target and strategy post 2020 are yet to be made. However, at Ban Ki-moon's Climate Summit in New York in September 2014, Mr. Alexander Bedritsky, the chair of the Russian delegation, suggested in his plenary speech ${ }^{2}$ that Russia's target towards 2030 could be to keep GHG emissions at $70-75 \%$ of 1990 levels. ${ }^{3}$ This is not an official commitment but rather a qualified opinion. Still, it offers some hints that give more grounds and certainty for the expectations and further actions in the sphere of climate change in Russia. 


\section{Russian companies' response to climate change: an overview of responses submitted to CDP}

\section{Responding companies}

Since 2003, CDP has requested annual climate change disclosures from major Russian companies ${ }^{1}$ of various sectors. The information is collected in a single format with the help of the CDP's Climate Change questionnaire, which may be filled out by companies using the CDP Online Response System.

In 2013, 15 Russian companies responded to the requests or volunteered to participate in the CDP Climate Change program. Out of this number, 10 companies submitted their information in the questionnaires and five companies referred to their data being included in the response submitted their data being included in the response submitted to CDP by their parent company. In 201414 companies participated in the CDP Climate Change program in Russia: nine companies responded to CDP and five companies provided information through another company's CDP response (see Figure 4).

Figure 4 Number of responding companies, by type of response

$A Q$ - Answered the Questionnaire (including late respondents)

SA - See Another: Companies provided data through another company's response.

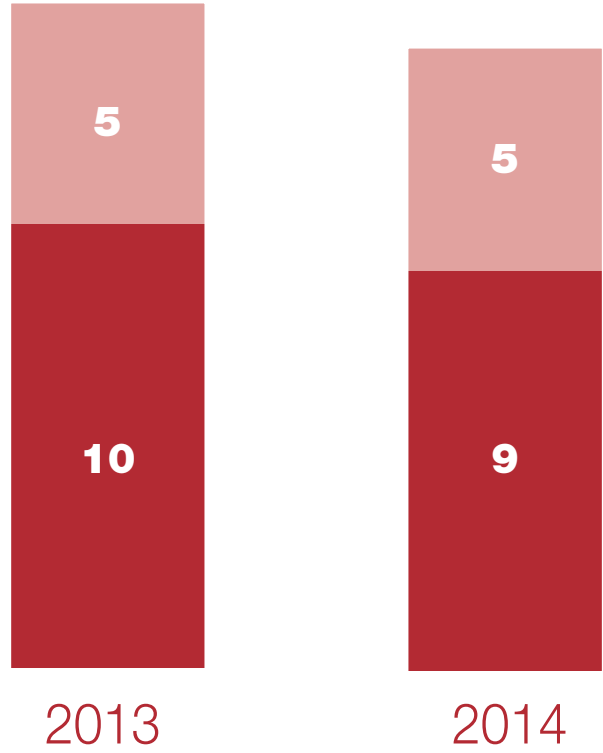

1 Russian companies herein mean companies registered in Russia or companies registered in other jurisdictions but operating in Russia.
These response rates are highest so far: the CDP Climate Change program has never attracted that many participants from Russia before ${ }^{2}$.

In general, the list of companies that have responded to the CDP climate change information request in 2014 hasn't changed much compared to the previous year (see Table 3). Of the companies that responded to the CDP in 2013, three companies chose not to provide information in 2014. There were also two new respondents in 2014, and one of those two provided information to CDP for the first time.

The long-time participants include, most notably, Gazprom and NOVATEK. These companies demonstrate their commitment to transparency and consistently provide their information in response to CDP's Climate Change program information requests. Arkhangelsk Pulp and Paper Mill responded to CDP for the first time in 2014 and provided a comprehensive response demonstrating commitment to managing climate change risks and to the implementation of relevant policies and procedures.

Responding companies represent a number of different sectors: energy, primarily oil and gas, materials, including metals, fertilisers and paper, and utilities (power producers and electric utilities), as well as financials.

\section{Key findings}

In general, many Russian companies are reluctant to participate in the CDP Climate Change information request and disclose information about their climate change-related activities and results. The overall numbers of Russian companies participating in the CDP in 2013 and 2014 are much lower compared to other developed and leading emerging economies, including Brazil, India, China and South Africa.

Low activity can be explained by various factors, including limited international investor reach into the Russian stock market, unavailability of carbon markets and Kyoto flexibility mechanisms to Russian companies and absence of an adequate national substitute for such markets and mechanisms, as well as the lack of provisions for mandatory corporate carbon accounting and reporting in the Russian legislation, and so on.

Russian companies' responses seldom present comprehensive information or a good analysis of climate aspects, corresponding risks and opportunities. Most companies remain silent on such topics as their climate change impacts and climate strategy. This is not unusual for countries where corporate GHG emissions accounting, reporting and management are not sufficiently developed. 
On the other hand, responding to CDP signals a commitment of the company to manage climate change risks and opportunities, and investors may consider this alone an indication of proactive sustainability management.

We can expect the number of responding companies in Russia to increase and the quality of their responses to improve as the companies recognize the benefits of participating in the CDP climate change program and develop their expertise in GHG emissions and climate risk management. This trend has already become visible. The number of companies responding directly to the CDP has grown over last few years, and the quality of responses by the comparable range of companies has notably improved in 2014 compared to 2013.

\section{Table 3 The list of the companies responding to CDP Climate Change program from Russia}

\begin{tabular}{|c|c|c|c|c|}
\hline & Company Name & Sector & 2013 & 2014 \\
\hline \multirow{12}{*}{$A Q^{*}$} & Arkhangelsk Pulp and Paper Mill & Materials & - & $\checkmark$ \\
\hline & EVRAZ PLC & Materials & $\checkmark$ & $\checkmark$ \\
\hline & Gazprom & Energy & $\checkmark$ & $\checkmark$ \\
\hline & Krasnoyarskaya GES & Utilities & $\checkmark$ & $\checkmark$ \\
\hline & Lukoil & Energy & - & $\checkmark$ \\
\hline & MOESK & Utilities & $\checkmark$ & - \\
\hline & NOVATEK & Energy & $\checkmark$ & $\checkmark$ \\
\hline & Surgutneftegas & Energy & $\checkmark$ & $\checkmark$ \\
\hline & TGK-1 & Utilities & $\checkmark$ & - \\
\hline & United Company RUSAL & Materials & $\checkmark$ & - \\
\hline & Uralkali & Materials & $\checkmark$ & $\checkmark$ \\
\hline & V. Bank - Vozrozhdenie Bank & Financials & $\checkmark$ & $\checkmark$ \\
\hline \multirow{5}{*}{$S A^{* *}$} & E.ON Russia & Utilities & $\checkmark$ & $\checkmark$ \\
\hline & Enel OGK-5 & Utilities & $\checkmark$ & $\checkmark$ \\
\hline & Fortum & Utilities & $\checkmark$ & $\checkmark$ \\
\hline & Raspadskaya & Energy & $\checkmark$ & $\checkmark$ \\
\hline & Wimm-Bill-Dann Foods & Consumer Staples & $\checkmark$ & $\checkmark$ \\
\hline
\end{tabular}

${ }^{*} \mathrm{AQ}-$ Companies that answered CDP questionnaire (including late respondents) 


\section{Climate change governance, risks and opportunities}

Our analysis of responses from companies in 2014 has identified some promising trends in the governance and decision making processes regarding climate issues. All companies reported that they have placed their climate change strategy under responsibility of the top management (senior manager/officer), member of the Board of Directors or a committee appointed by the Board. Detailed evaluation shows that climate change issues are the responsibility of executives responsible for environmental protection or overall health, safety and environment, energy efficiency, quality, or of top executives overseeing emission trading among other topics (see Figure 5).

In their 2014 submissions, the majority of the responding companies (55\%) emphasized climate change-related risks driven by expected introduction of mandatory GHG emissions monitoring and reporting. Some companies consider as risks to them the possibility of introduction of GHG emissions limits (quotas) or carbon taxes. These concerns have been, apparently, raised by the Russian Government's activity on climate change mitigation in 2013 and 2014 (see previous section for details). Legal risks that may arise from the adoption of new regulations relating to the Russian Federation's involvement in the global binding climate change agreements should be attributed to the same category.
Most of the responding companies (89\%) state in their answers that they incorporate climate change issues into their general business strategy. It is important to note that these are not mere claims; respondents describe approaches they use to address specific climate change risks. Still some responding companies do not have documented processes and procedures for assessing and managing climate change risks and opportunities, and some are just starting implementation of $\mathrm{GHG}$ reduction initiatives (see Figure 6).

Several companies have implemented specific instruments for GHG emissions management. In addition to the ISO 14001 environmental management system providing overall framework for implementing environmental policy, some responding companies also use the ISO 14064 standard for greenhouse gas accounting and management.

Just over half of the responding companies identified and declared measurable absolute and/or intensity GHG emissions reduction targets. The remaining companies, apparently, limit their climate change management activities to general environmental procedures; their current position can be characterized as being on the "business as usual" route.

The most common GHG emissions reduction measures taken by the companies include projects to limit flaring of associated petroleum gas by increasing its utilization (typical for of oil and gas production companies), and implementation of energy efficiency retrofit programs

Figure 5 Allocation of the responsibility for climate change among decision makers, \% of the total number of the respondents

\section{3}

20\% Environmental Management

$30 \%$ Health, Safety and Environmental Management

10\% Quality, Health, Safety and Environment

$20 \%$ CEO, Deputy CEO or Senior VP

10\% Energy Efficiency and Environmental protection

$10 \%$ Not specified

\section{4}

22\% Environmental Management

34\% Health, Safety and Environmental Management

11\% Quality, Health, Safety and Environment

$22 \%$ CEO, Deputy CEO or Senior VP

11\% Energy Efficiency and Environmental protection

$0 \%$ Not specified
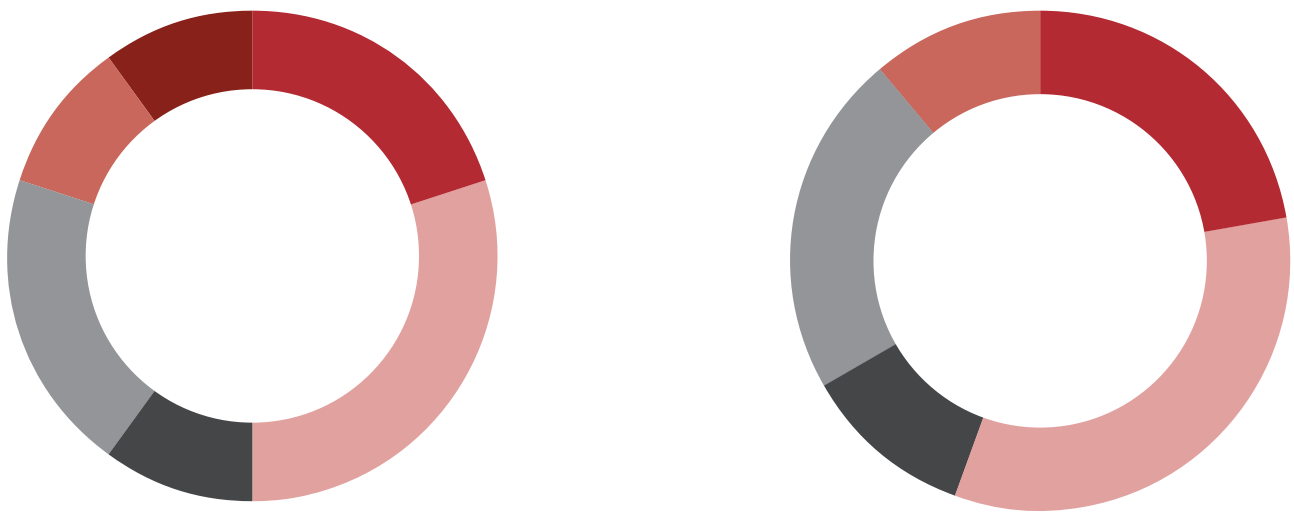
based on operations development strategies and third party energy audit recommendations (common for all responding companies).

\section{Emissions reporting and external verification}

Many Russian companies prepare and publish GHG emissions data. Of the CDP Climate Change program respondents in Russia in 2014, 78\% reported Scope 1 emissions, and $67 \%$ provided Scope 2 data. However, in most cases the GHG emission assertions of Russian companies and public reports containing them are not independently verified. This general observation was confirmed by responses to CDP questionnaires in 2014, which indicated that 33\% of the participating Russian companies used third party assurance.

The main drivers for Russian companies to obtain external verification are providing verified data for their public sustainability reports, requirements and audits ${ }^{1}$ of public authorities, and meeting of ISO 14064 requirements. Interestingly, participation in emission trading schemes, particularly EU Emissions Trading Scheme (ETS) for companies with assets subject to EU requirements is not a driver for external verification of GHG emissions. Not a single company has identified this factor in their responses.
Given the current efforts to establish a regulatory framework for GHG emissions management and reduction in Russia, it seems reasonable to expect that the Russian companies' interest in carbon verification and assurance would increase in the coming years.

\section{Company scores}

The company responses submitted were scored using the CDP methodology ${ }^{2}$. Companies receive a CDP climate disclosure score and, if their disclosure level is sufficient, a CDP performance score (See Table 4 on page 22). The scoring approach is explained in this report.

Russian companies' scores for 2013 and 2014 are presented in Table 4 on page 22.

In 2014, Arkhangelsk Pulp and Paper Mill received the highest score, suggesting that they are the most transparent company with regards to climate change disclosure in Russia.

Figure 6 Snapshot of companies' climate change risk management procedures, \% of the total number of the respondents

\section{3}

78\% Integrated into multidisciplinary company wide risk management processes

22\% There are no documented processes for assessing and managing risks and opportunities from climate change

0\% A specific climate change risk management process

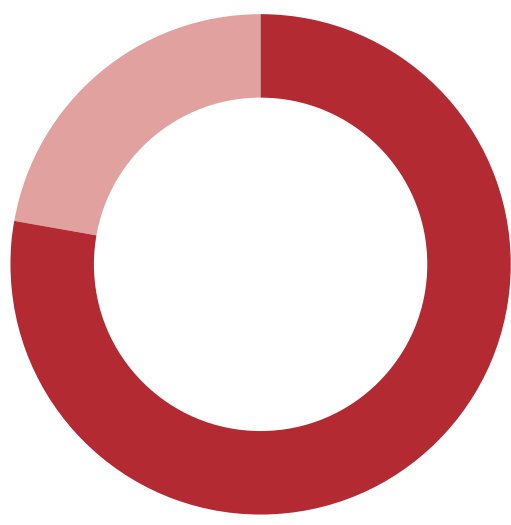

1 Used to be a requirement in Russia for GHG emission reports developed within the framework of $\mathrm{JI}$ project implementation as provided for by Article 6 of the Kyoto Protocol.

\section{4}

62\% Integrated into multidisciplinary company wide risk management processes

25\% There are no documented processes for assessing and managing risks and opportunities from climate change

13\% A specific climate change risk management process

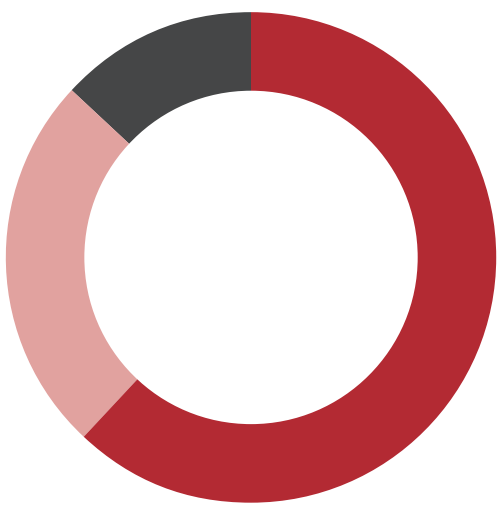

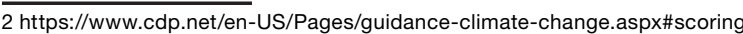




\section{Scoring results}

GDP RUSSIA 2014 COMPANY WITH THE HIGHEST DISCLOSURE SCORE

Arkhangelsk Pulp and Paper Mill

Paper Products

\begin{tabular}{|c|c|c|}
\hline Company & $\begin{array}{c}\text { CDP Score } \\
2013\end{array}$ & $\begin{array}{c}\text { CDP Score } \\
2014\end{array}$ \\
\hline Arkhangelsk Pulp and Paper Mill & - & $77 \mathrm{C}$ \\
\hline Gazprom & $62 \mathrm{C}$ & $66 \mathrm{C}$ \\
\hline Krasnoyarskaya GES & - & $63 \mathrm{E}$ \\
\hline Evraz & 22 & $62 \mathrm{E}$ \\
\hline Novatek & 40 & $50 \mathrm{E}$ \\
\hline Uralkali & 41 & 40 \\
\hline Surgutneftegas & 23 & 23 \\
\hline V. Bank - Vozrozhdenie Bank & - & 22 \\
\hline Lukoil & - & 16 \\
\hline
\end{tabular}



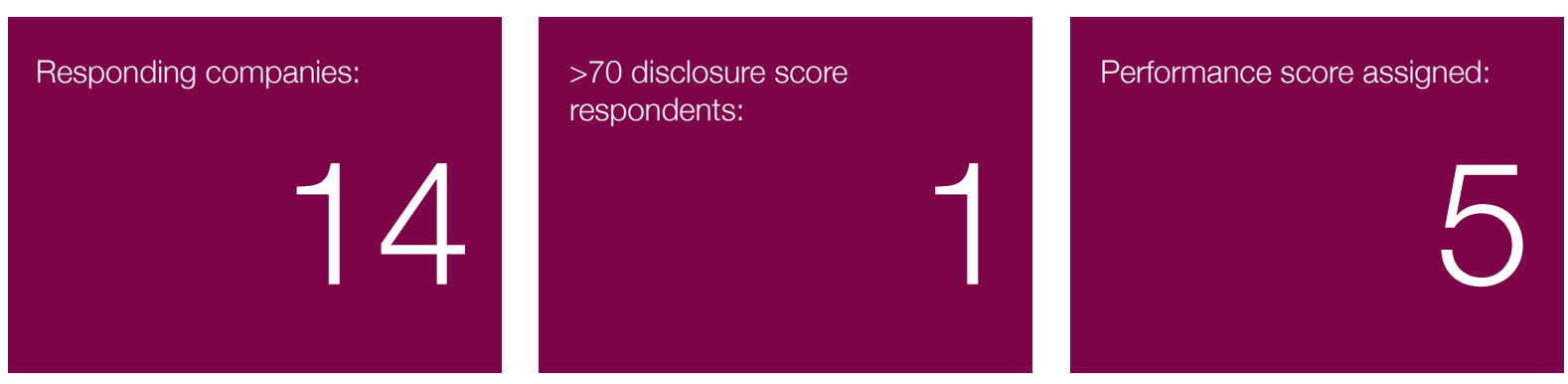

Response

and Scoring

Summary
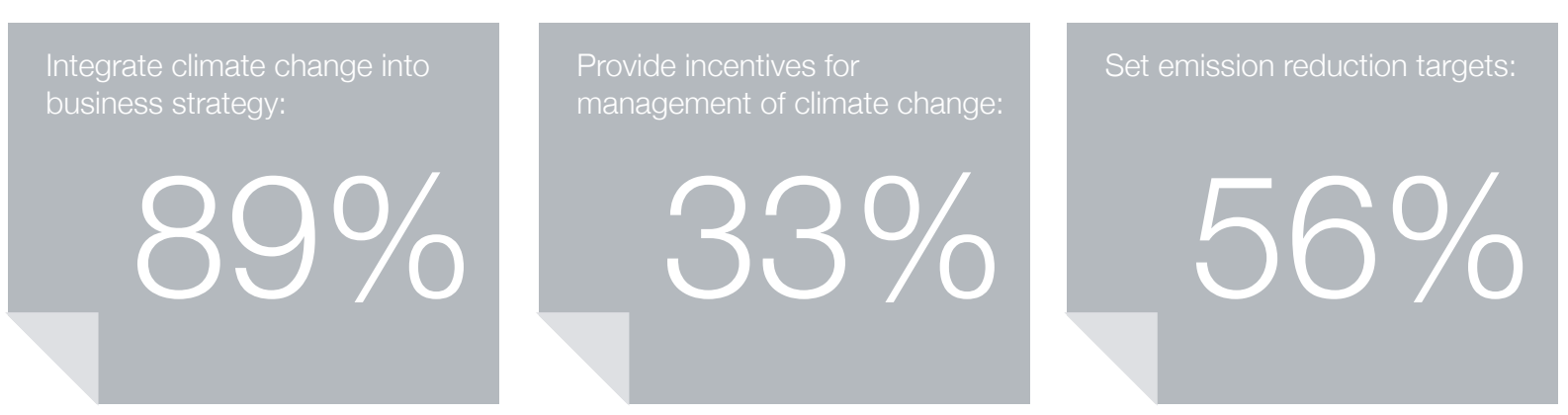

Top risks:

Risks driven by changes in regulation

Risks driven by changes in physical climate parameters
Top opportunities:

Opportunities driven by changes in regulation

Opportunities driven by changes in other climaterelated developments

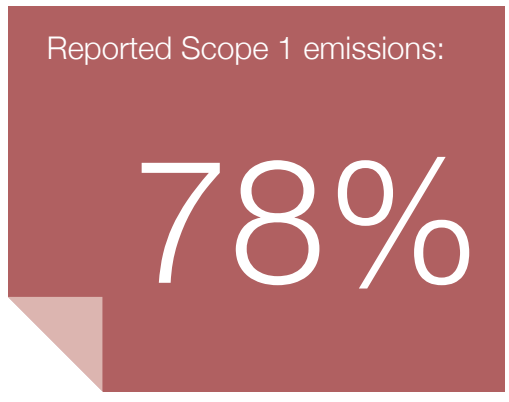

Reported both absolute and intensity emissions targets:

$$
11 \%
$$
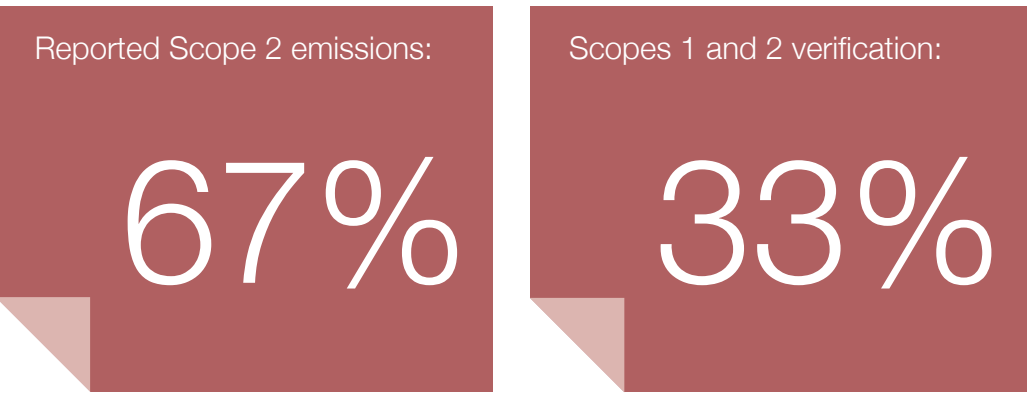

Reported absolute targets only:

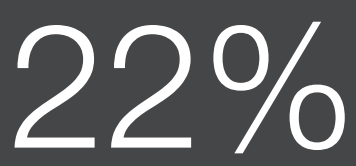

Risks \&

Opportunities

Emissions Reporting

Reported intensity targets only:

Emission

Reduction

Targets 
Understanding CDP disclosure scores and performance bands

Relative weight of elements in determining the disclosure score

The disclosure score assesses the completeness and quality of a company's response. Its purpose is to provide a summary of the extent to which companies have answered CDP's questions in a structured format. A high disclosure score signals that a company provided comprehensive information about the measurement and management of its carbon footprint, its climate change strategy and risk management processes and outcomes.

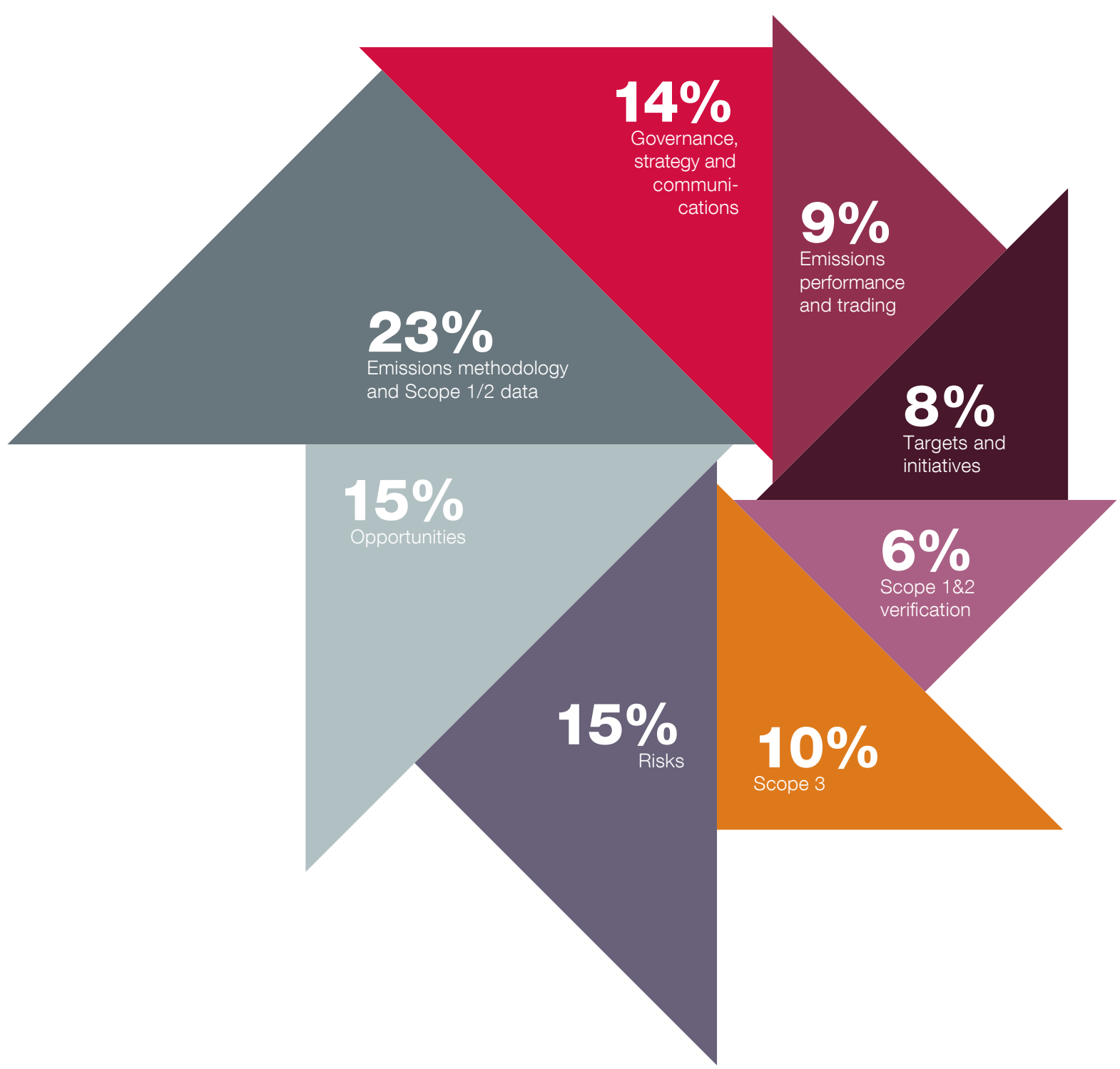

\section{How the disclosure score is calculated}

Disclosure points earned

$\times 100=$ Disclosure score $\%$

$>\mathbf{5 0} \%$ disclosure $=$ scored for performance

Disclosure points available

0 to 100 


\section{Relative weight of elements in determining the performance bands}

The performance score assesses the level of action, as reported by the company, on climate change mitigation, adaptation and transparency. Its intent is to highlight positive climate action as demonstrated by a company's CDP response. A high performance score signals that a company is measuring, verifying and managing its carbon footprint, through actions such as setting and meeting carbon reduction targets and implementing programs to reduce emissions in both its direct operations and supply chain.

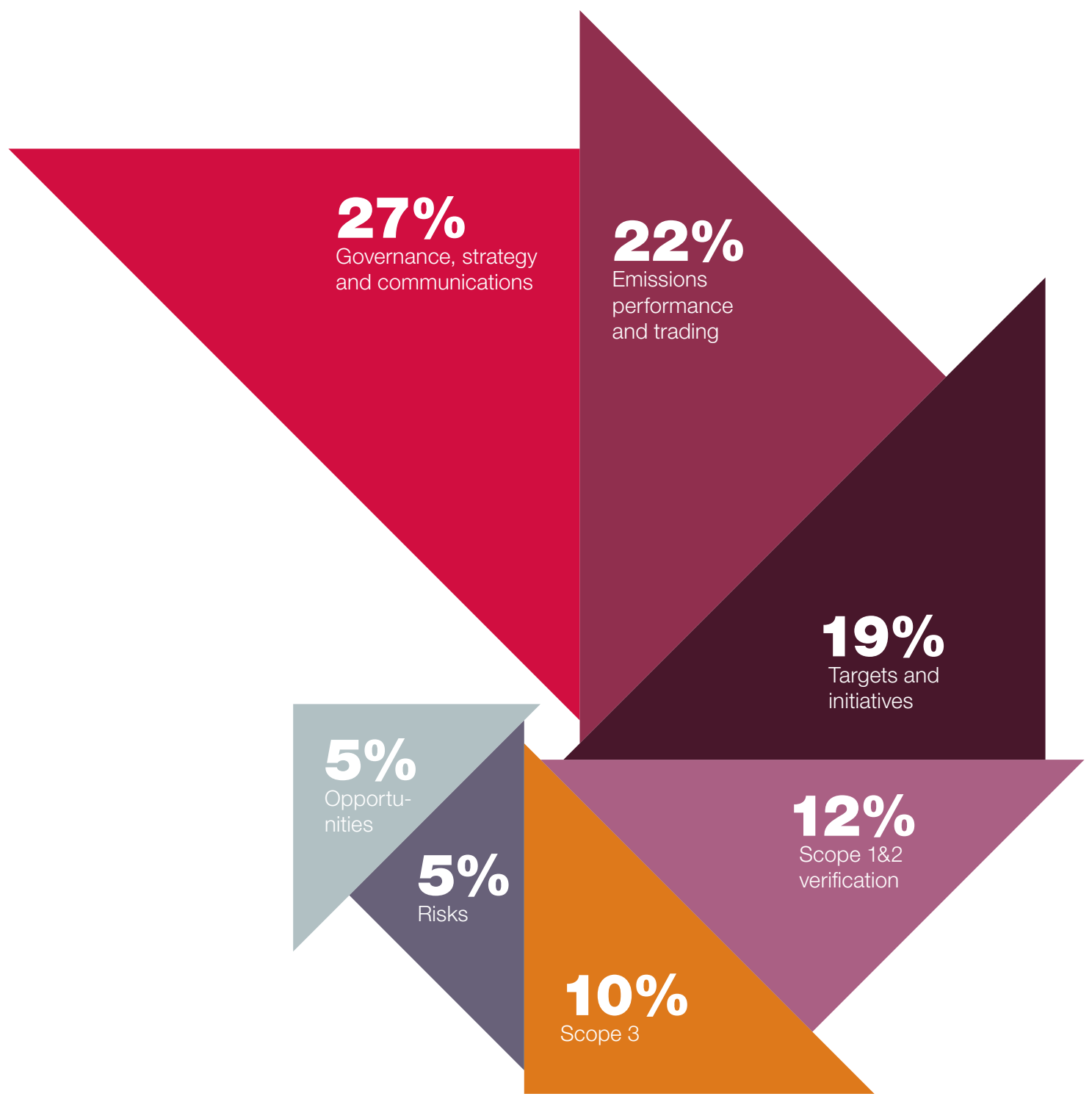

\section{How the performance score is calculated}

Performance points earned

Performance points available $\times 100=$ Performance score $\%=$ Performance band

\section{A to $E$}




\section{Russian companies' response to CDP Climate Change program in 2013 and 2014}

\begin{tabular}{|c|c|c|c|c|c|c|c|c|c|}
\hline Company Name & 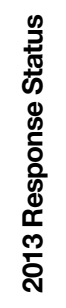 & 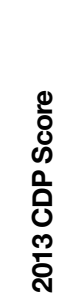 & 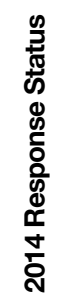 & 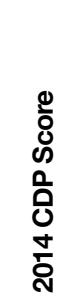 & Company Name & 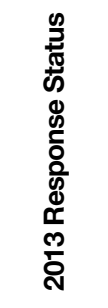 & 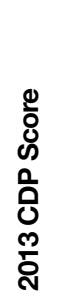 & 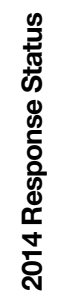 & 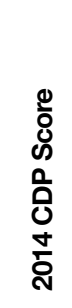 \\
\hline Consumer Discretionary & & & & & Open Investments (OPIN) & NR & & $\mathrm{Nl}$ & \\
\hline Avtovaz & NR & & $\mathrm{Nl}$ & & PIK Group & NR & & NR & \\
\hline M Video & NR & & NR & & Rosbank & NR & & NR & \\
\hline Sollers & NR & & NR & & Sberbank & NR & & NR & \\
\hline Consumer Staples & & & & & V. Bank (Bank Vozrozhdenie) & $A Q(L)$ & * & $A Q$ & 22 \\
\hline Dixy Group & NR & & NR & & VTB Bank & NR & & NR & \\
\hline KBK Cheryomushki & NR & & $\mathrm{Nl}$ & & Health Care & & & & \\
\hline Magnit & DP & & DP & & Pharmstandard & NR & & NR & \\
\hline Wimm-Bill-Dann Foods & SA & & SA & & Industrials & & & & \\
\hline Energy & & & & & Aeroflot & DP & & DP & \\
\hline Bashneft & DP & & DP & & Kamaz & $\mathrm{NI}$ & & NR & \\
\hline Exillon Energy & NR & & NR & & Mostotrest & NR & & DP & \\
\hline Gazprom & $A Q$ & $62 \mathrm{C}$ & $A Q$ & $66 \mathrm{C}$ & Novorossiysk Commercial Sea Port & DP & & DP & \\
\hline Gazprom Neft & NR & & NR & & Svetlana & $\mathrm{NI}$ & & NR & \\
\hline Lukoil & DP & & $A Q$ & 16 & Teploobmennik PDC & $\mathrm{NI}$ & & NR & \\
\hline Novatek & $A Q$ & 40 & $A Q$ & $50 \mathrm{E}$ & Tomskenergoremont & $\mathrm{NI}$ & & NR & \\
\hline Raspadskaya & SA & & SA & & Trest Gidromontag & $\mathrm{NI}$ & & NR & \\
\hline RN Holding & $\mathrm{NI}$ & & NR & & Tupolev PSC & $\mathrm{NI}$ & & NR & \\
\hline Rosneft & NR & & NR & & UTair Aviation & NR & & NR & \\
\hline RusPetro & DP & & DP & & Vanino Commercial Sea Port & $\mathrm{NI}$ & & NR & \\
\hline Slavneft & $\mathrm{NI}$ & & $N R$ & & Materials & & & & \\
\hline Surgutneftegas & $A Q$ & 23 & $A Q$ & 23 & Acron & DP & & DP & \\
\hline Tatneft & NR & & NR & & Alrosa Company Ltd & NR & & NR & \\
\hline TNK-BP Holding & NR & & NR & & Arkhangelsk Pulp and Paper Mill & $\mathrm{NI}$ & & $A Q$ & $77 \mathrm{C}$ \\
\hline Transneft & NR & & NR & & Evraz PLC & $A Q$ & 22 & $A Q$ & $62 \mathrm{E}$ \\
\hline Udmurtneft & $\mathrm{NI}$ & & NR & & Koks & $\mathrm{NI}$ & & NR & \\
\hline Ufaneftekhim & NR & & $N R$ & & Mechel & DP & & DP & \\
\hline Yamal LNG & $\mathrm{NI}$ & & DP & & MMC Norilsk Nickel & DP & & DP & \\
\hline Financials & & & & & $\begin{array}{l}\text { MMK (Magnitogorsk Iron \& Steel } \\
\text { Works) }\end{array}$ & NR & & NR & \\
\hline Bank of Moscow (Bank Moskvy) & NR & & $N R$ & & Novolipetsk Steel (NLMK) & DP & & DP & \\
\hline Bank St Petersburg & NR & & NR & & Petropavlovsk Plc & DP & & DP & \\
\hline LSR Group & NR & & NR & & Phosagro & NR & & NR & \\
\hline Moscow Exchange MICEX-RTS & $\mathrm{NI}$ & & NR & & Pigment Holding Company & NR & & $\mathrm{NI}$ & \\
\hline
\end{tabular}




\begin{tabular}{|c|c|c|c|c|}
\hline Company Name & 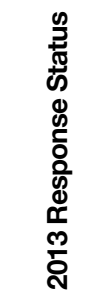 & 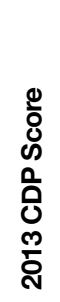 & 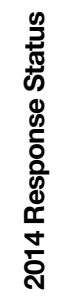 & 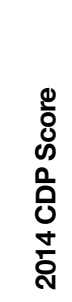 \\
\hline Polymetal & DP & & DP & \\
\hline Polyus Gold & NR & & NR & \\
\hline Severstal & NR & & NR & \\
\hline Sibirskiy Cement & $\mathrm{NI}$ & & NR & \\
\hline TMK & $\mathrm{NI}$ & & DP & \\
\hline United Company RUSAL & $\mathrm{AQ}(\mathrm{L})$ & * & NR & \\
\hline Uralkali & $A Q$ & 41 & $A Q$ & 40 \\
\hline Uuzhuralnikel & $\mathrm{Nl}$ & & NR & \\
\hline VSMPO AVISMA & NR & & NR & \\
\hline \multicolumn{5}{|l|}{ Telecommunication Services } \\
\hline AFK Sistema & DP & & DP & \\
\hline MegaFon & $\mathrm{NI}$ & & NR & \\
\hline Mobile TeleSystems & NR & & NR & \\
\hline Rostelecom & NR & & NR & \\
\hline VimpelCom Ltd & NR & & NR & \\
\hline \multicolumn{5}{|l|}{ Utilities } \\
\hline Bashkirenergo & NR & & NR & \\
\hline E.ON Russia & SA & & SA & \\
\hline Enel OGK-5 & SA & & SA & \\
\hline Far Eastern Energy Company & $\mathrm{NI}$ & & NR & \\
\hline FGC UES & NR & & NR & \\
\hline Fortum & SA & & SA & \\
\hline INTER RAO UES & DP & & DP & \\
\hline Irkutskenergo & NR & & NR & \\
\hline Kamchatskenergo & NR & & NR & \\
\hline Krasnoyarskaya GES & $A Q(L)$ & * & $A Q$ & $63 \mathrm{E}$ \\
\hline Kuzbassenergo & NR & & NR & \\
\hline MOESK & $A Q(L)$ & * & NR & \\
\hline Mosenergo & NR & & NR & \\
\hline Novosibirskenergo & $\mathrm{NI}$ & & NR & \\
\hline OGK-1 & NR & & NR & \\
\hline OGK-2 & NR & & NR & \\
\hline OGK-6 & $\mathrm{NI}$ & & NR & \\
\hline
\end{tabular}

\begin{tabular}{|c|c|c|c|}
\hline Company Name & 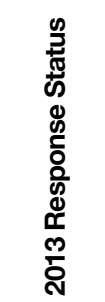 & 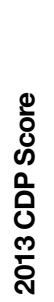 & 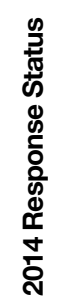 \\
\hline $\begin{array}{l}\text { Quadra - Power Generation } \\
\text { Company }\end{array}$ & NR & & DP \\
\hline Rosseti (Russian Grids) & NR & & NR \\
\hline RusHydro & DP & & DP \\
\hline TGC-1 & $A Q(L)$ & * & NR \\
\hline TGC-5 & $N R$ & & NR \\
\hline TGC-6 & NR & & NR \\
\hline TGC-9 & NR & & NR \\
\hline Volga (Volzhskaya) TGC & NR & & NR \\
\hline WGC-3 (OGC-3) & NR & & NR \\
\hline Yenisei TGC (TGC-13) & NR & & NR \\
\hline
\end{tabular}

$A Q$ - Answered Questionnaire

$A Q(L)$ - Answered Questionnaire (Late)

DP - Declined to Participate

$\mathrm{NI}$ - Not Invited to Participate

NR - Not Responded

SA - See Another - provided data through another company's response

$\left(^{\star}\right)$ - Company not scored due to late submission 


\section{Sabanc1 Universites1

Sponsor

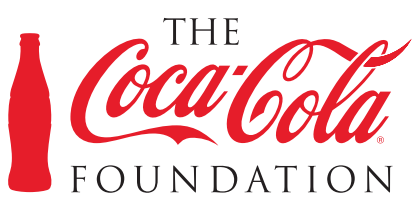

FOUNDATION

CDP Russia Local Support Partner

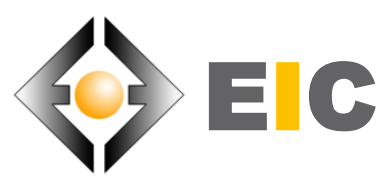

\section{Important Notice}

The contents of this report may be used by anyone providing acknowledgement is given to CDP Worldwide (CDP). This does not represent a license to repackage or resell any of the data reported to CDP or the contributing authors and presented in this report. If you intend to repackage or resell any of the contents of this report, you need to obtain express permission from CDP before doing so.

EY, Sabanci University and CDP have prepared the data and analysis in this report based on responses to the CDP 2014 information request. No representation or warranty (express or implied) is given by EY, Sabanci University or CDP as to the accuracy or completeness of the information and opinions contained in this report. You should not act upon the information contained in this publication without obtaining specific professional advice. To the extent permitted by law, EY, Sabanci University and CDP do not accept or assume any liability, responsibility or duty of care for any consequences of you or anyone else acting, or refraining to act, in reliance on the information contained in this report or for any decision based on it. All information and views expressed herein by CDP and/or EY, and/or Sabanci University is based on their judgment at the time of this report and are subject to change without notice due to economic, political, industry and firm-specific factors. Guest commentaries where included in this report reflect the views of their respective authors; their inclusion is not an endorsement of them.

EY, Sabanci University and CDP and their affiliated member firms or companies, or their respective shareholders, members, partners, principals, directors, officers and/or employees, may have a position in the securities of the companies discussed herein. The securities of the companies mentioned in this document may not be eligible for sale in some states or countries, nor suitable for all types of investors; their value and the income they produce may fluctuate and/or be adversely affected by exchange rates.

CDP Worldwide' and 'CDP' refer to CDP Worldwide, a United Kingdom company limited by guarantee, registered as a United Kingdom charity number 1122330

(c) 2014 CDP Worldwide. All rights reserved. 
CDP Contacts

\section{Sue Howells}

Co-Chief Operating Officer

\section{Daniel Turner}

Head of Disclosure

\section{James Hulse}

Head of Investor Initiatives

\section{Antigone Theodorou}

Director, Global Operations

\section{CDP}

CDP Head Office

3rd Floor, Quadrant House

4 Thomas More Square

Thomas More Street

London, E1W 1YW

United Kingdom

Tel: +44 (0) 2038183900

www.cdp.net

info@cdp.net
Partner Contacts

Melsa Ararat

Director

\section{Mirhan Köroğlu Göğüş}

Project Manager

\section{Sabanci University}

Orhan Tuzla 34956

Istanbul

Turkey

Tel: +90 (0) 2164839682

copturkey.sabanciuniv.edu

cdp@sabanciuniv.edu
Local Partner Contacts

Michael Yulkin

EIC

Vladimir Dyachkov

EIO

Yulia Schastnaya

EIO

Valeria Moskvina

$\mathrm{EIC}$

\section{Sergey Dayman}

EY Russia

Kostiantyn Taranets

EY Russia
Report Writer Contacts

Michael Yulkin

EIC

Sergey Dayman

EY Russia

Kositantyn Taranets

EY Russia

Melsa Ararat

CDP Turkey

Our sincere thanks are extended to the following indivuduals for reviewing Russia's Climate Profile and Key Trends section:

\section{Boris Porfiriev}

Russian Academy of Sciences

\section{Alexey Kokorin}

WWF Russia 


вИวЈо $入$ 入

пәнеdе $\perp$ ницнецоноу

виоวо

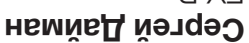

$\operatorname{sи\Pi dK\perp dOO~}$

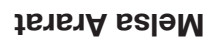

виоวод $ᄉ \exists$

пәнеdе $\perp$ ницнецоно्र

виวо๐ неwие $\square$ йәЈ⿰әว

иєП

нихч어 чиехиш

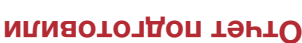

ИЕП енияхооW ьиdәrеg

иєП

венцоеһо вич어

иєП

gохиват dиwиterg

иєП

нихч어 เиехиพ

ииวооd

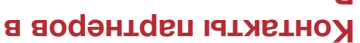

เәu'dpoøołu!

tәu'dpo'MMM

006ع8 เ8ع0乙 (0) $\nabla \nabla+: \perp$

винециdgохицәg

ML MLヨ 'HOZHOS edow eoewo $\perp$ епицК

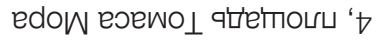
оКех $\perp$ неdъеgУ 'жецє йи- $\varepsilon$ оифо ионаоцо 」dQO

dao

Иะวончเәцьอไ

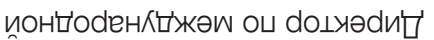

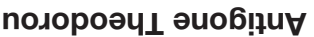

npə'A!̣un!̣ueqes@dpo

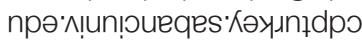
乙896 ع8† 9 เ乙 (0) 06+ : । ви' $\mathrm{d} \Lambda_{\perp}$ '

$9967 \varepsilon$ e $\left\llcorner\varepsilon K_{\perp}\right.$ HexdO иһнеgеว เәциэdәgинК

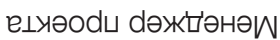

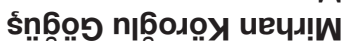

dоцуәдй ңелель еs|әW

иwedoıวəяни ว әцоged оப

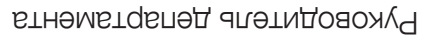
әs|nH səuाer

иипеwdoфни оиицаdу্ере оப

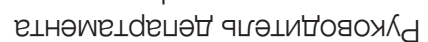

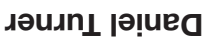

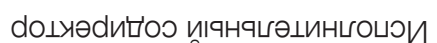
s॥әмоH әns

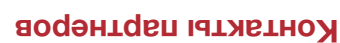

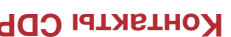




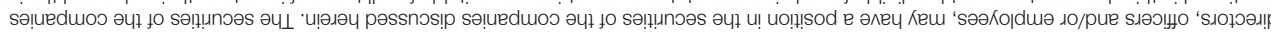

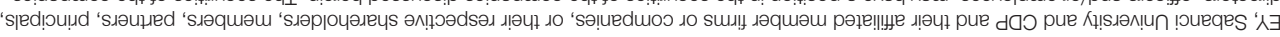

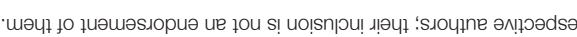

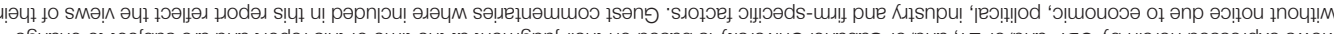

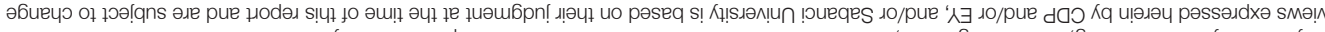

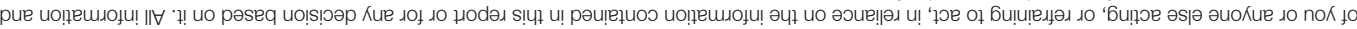

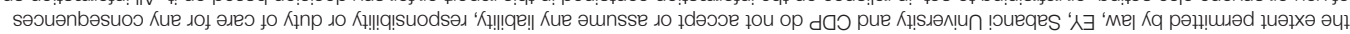

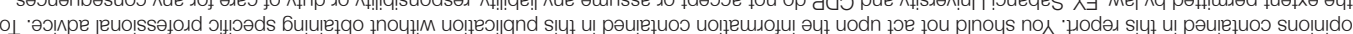

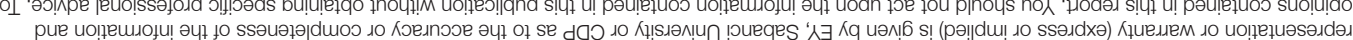

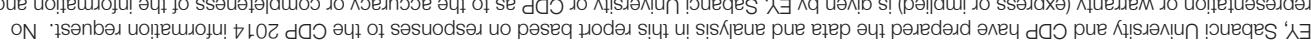

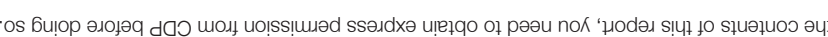

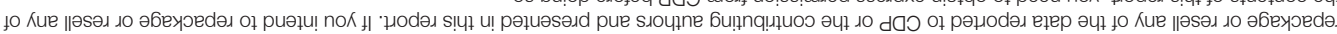

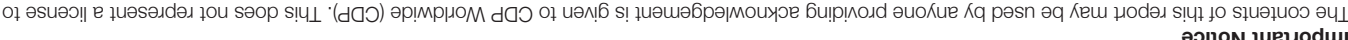




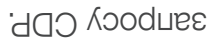

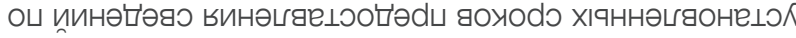

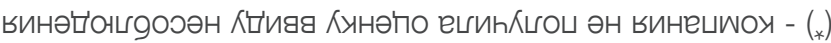

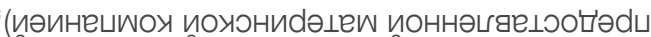

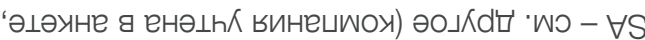

$$
\text { ' } \mathrm{\perp \partial \theta \perp O} \perp \partial \mathrm{H}-\mathrm{UN}
$$

'dOO

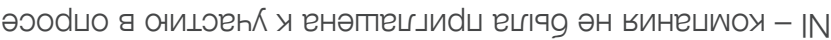

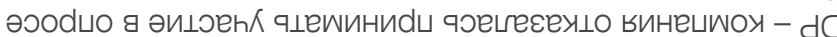

'(พәинец'єочо

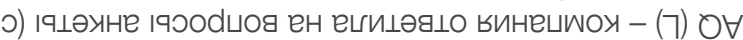

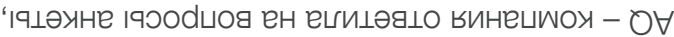

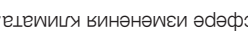

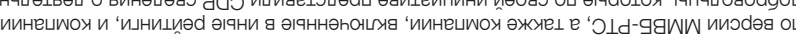

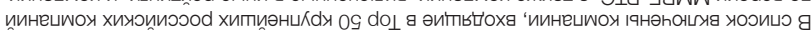

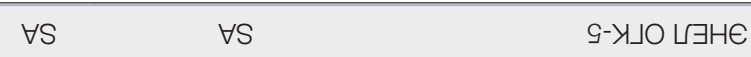

$\forall S \quad \forall S \quad$ виоวоd Ho $\Theta$

पू $\quad$ पN

$\forall S \quad \forall S \quad$ WKıdoథ

प्dN $\quad$ पN 6-Y्र」

प्CN $\quad$ प्CN $9-Y \perp \perp$

\begin{tabular}{|c|}
\hline yN $\quad$ g-y」J \\
\hline
\end{tabular}

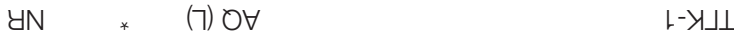

\begin{tabular}{|c|c|}
\hline $\mathrm{dO}$ & $\mathrm{dO}$ \\
\hline
\end{tabular}

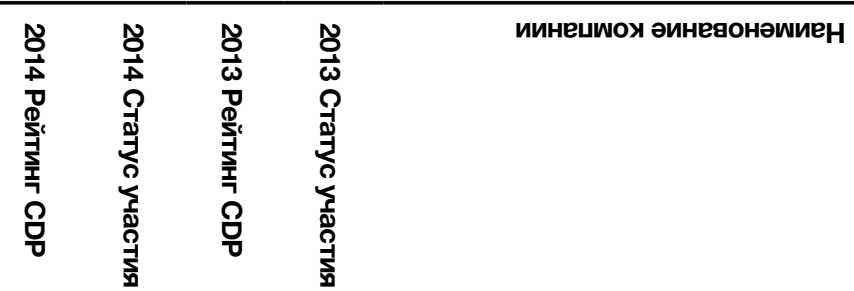

YN

yN

иเəวЈ০d

(1)

yN

IN

9-Ү्र」O

प्N $\quad$ YN

$\varepsilon-\Upsilon \supset\lrcorner O$

पN $\quad$ CN

乙-Ү्र」O

प्N $\quad$ UN

प्रN IN

$\llcorner-Y \mid\lrcorner O$

о」dәнєеуойиоояон

YN $\quad * \quad$ (7) $\mathrm{OV}$

YOEOW

प्dN $\quad$ पN

o」dəнєอоW

पN $\quad$ yN

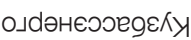

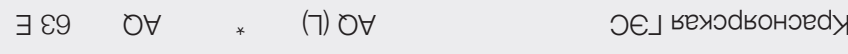

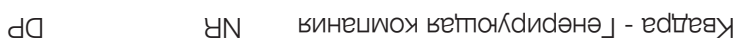

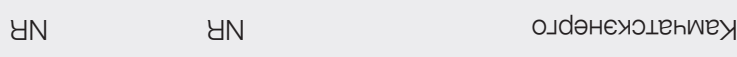

प्रN $\quad$ ON

da $d 0$

Ј $\mathrm{O} \forall d \mathrm{~d} \exists \perp H И$

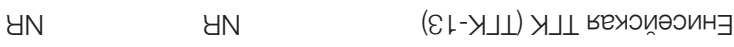

पN

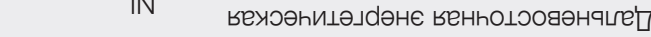

प्रN $\quad$ प्रN

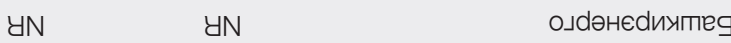

ехицәлdәнеодıхәге

\begin{tabular}{|c|c|c|}
\hline teN & पaN & પ્રнеgdəgО \\
\hline taN & पN & શ્રнеgэо d \\
\hline IN & yN & HИL \\
\hline
\end{tabular}

पIN IN OLd-9gWW ежdng веховохооW

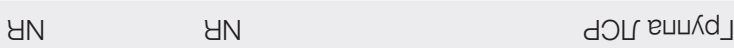

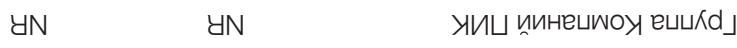

प्रN $\quad$ पN

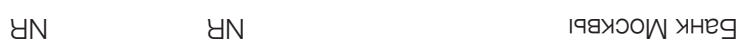

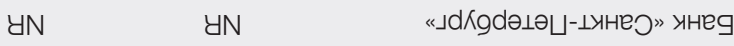

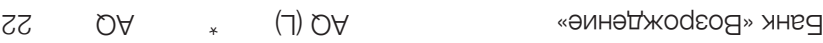

иЈКนгК әІवяоэнениФ

\begin{tabular}{|c|c|c|}
\hline yN & पN & 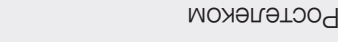 \\
\hline yN & yN & 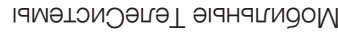 \\
\hline yN & IN & НОФе」әW \\
\hline $\mathrm{yN}$ & $\mathrm{dN}$ & พOY્રธəபWIqด \\
\hline
\end{tabular}

\begin{tabular}{|c|c|c|c|c|}
\hline 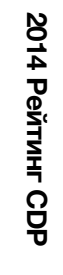 & 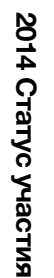 & 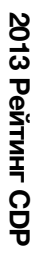 & 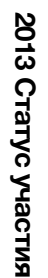 & иинешшох әинеяонәшиен \\
\hline
\end{tabular}




\begin{tabular}{|c|c|c|c|c|}
\hline & \multicolumn{2}{|l|}{ dO } & $d O$ & «еพəцЈиว" \\
\hline & \multicolumn{4}{|r|}{ 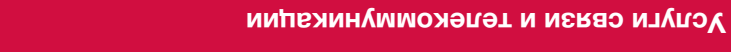 } \\
\hline & \multicolumn{2}{|l|}{ प्dN } & IN & же $\perp$ HOWOd $\amalg и\lrcorner \perp \mathrm{J}_{\perp}$ \\
\hline & \multicolumn{2}{|l|}{ yN } & IN & 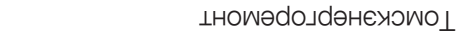 \\
\hline & \multicolumn{2}{|l|}{ पdN } & IN & енецәяว \\
\hline & \multicolumn{2}{|l|}{ yN } & IN & 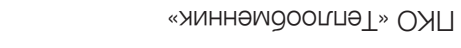 \\
\hline & \multicolumn{2}{|l|}{ yN } & IN & 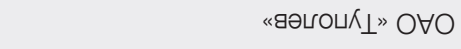 \\
\hline & \multicolumn{2}{|l|}{$d 0$} & $d 0$ & 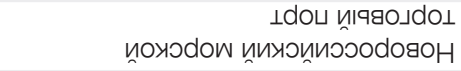 \\
\hline & \multicolumn{2}{|l|}{ dO } & पdN & 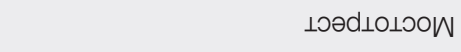 \\
\hline & \multicolumn{2}{|l|}{ पdN } & IN & $\varepsilon \forall W \forall X$ \\
\hline & \multicolumn{2}{|l|}{ पdN } & IN & 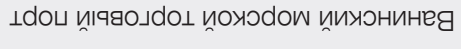 \\
\hline & \multicolumn{2}{|l|}{$d O$} & $d O$ & ıoscpode $\forall$ \\
\hline & \multicolumn{2}{|l|}{ पdN } & प्dN & «dиє $\perp$ ' ьинечพохеия $\forall$ \\
\hline \multicolumn{5}{|c|}{ 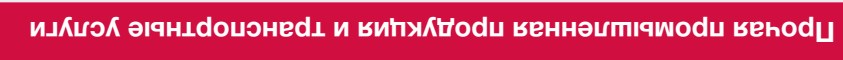 } \\
\hline & \multicolumn{2}{|l|}{ yN } & पn & วdəเธนо๐ \\
\hline & \multicolumn{2}{|l|}{ yN } & पdN & оәயия'W \\
\hline & \multicolumn{2}{|l|}{ IN } & पdN & $\varepsilon \forall$ Е๐ฯ४ \\
\hline & \multicolumn{4}{|c|}{ 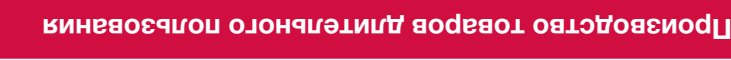 } \\
\hline & \multicolumn{2}{|l|}{$d 0$} & $d O$ & цинцеш \\
\hline & \multicolumn{2}{|l|}{ IN } & yN & 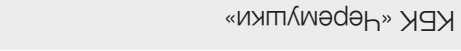 \\
\hline & \multicolumn{2}{|l|}{ yN } & प्dN & பபКd」 ИОУИЦ' \\
\hline & \multicolumn{2}{|l|}{$\forall S$} & $\forall S$ & 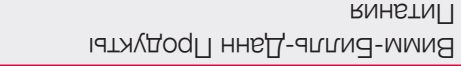 \\
\hline & \multicolumn{4}{|c|}{ 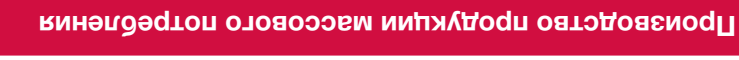 } \\
\hline & \multicolumn{2}{|c|}{ yn } & IN & qцәхиньгеdКжО人 \\
\hline & \multicolumn{2}{|l|}{ IN } & yN & 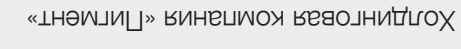 \\
\hline & \multicolumn{2}{|l|}{ yN } & प & od $\lrcorner \forall \supset O \Phi$ \\
\hline \multirow[t]{7}{*}{ Ot } & $O \forall$ & $L t$ & $O \forall$ & ийгеучгеdx \\
\hline & \multicolumn{2}{|l|}{$d 0$} & IN & $Y Y W \perp$ \\
\hline & \multicolumn{2}{|l|}{ प्dN } & IN & 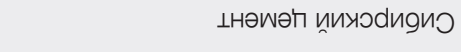 \\
\hline & \multicolumn{2}{|l|}{ प्dN } & पdN & 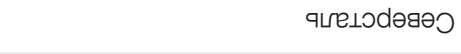 \\
\hline & $\forall S$ & & $\forall S$ & веуотечое d \\
\hline & yN & & पdN & 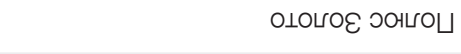 \\
\hline & $d 0$ & & $d O$ & เレгецәพицоப \\
\hline
\end{tabular}

\begin{tabular}{|c|c|c|c|c|}
\hline & yN & * & (ᄀ) $\mathrm{OH}$ & 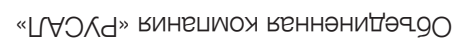 \\
\hline & dO & & dO & Y्रWLH \\
\hline & $d 0$ & & $d O$ & LəhəW \\
\hline & पdN & & पdN & 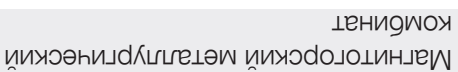 \\
\hline & UN & & yN & 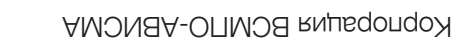 \\
\hline & yN & & IN & ગ્રО)્ર \\
\hline$\exists 乙 9$ & $O \forall$ & 乙乙 & $O \forall$ & $\varepsilon \forall d g \exists$ \\
\hline & $d 0$ & & $d O$ & 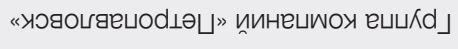 \\
\hline & $d 0$ & & $d O$ & «qьәуин ииихО৭циdoH" \\
\hline O LL & $O \forall$ & & IN & 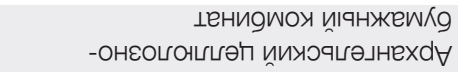 \\
\hline & yN & & yN & $\forall O O d \sqcup \forall$ \\
\hline & dO & & dO & Hody $\forall$ \\
\hline & & & иhะ्रKto & 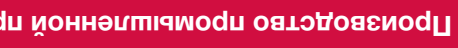 \\
\hline & yN & & $\mathrm{dN}$ & $\perp$ de'tHe $\perp$ owde $\Phi$ \\
\hline & & & ииһхК: & 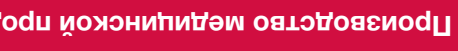 \\
\hline & $d 0$ & & IN & 」பО-เеพь \\
\hline & yN & & yN & ижப'dәнє ноьऽиэує \\
\hline & yN & & प्dN & кихәцфәнефк \\
\hline & yN & & IN & q ффән $\perp d K w \amalg K$ \\
\hline & yN & & पdN & वцфәнонед $\perp$ \\
\hline & प्dN & & $\mathrm{dN}$ & 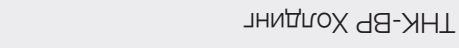 \\
\hline & yN & & $\mathrm{yN}$ & ૧ фрәнце $\perp$ \\
\hline$\varepsilon 己$ & $O \forall$ & $\varepsilon 己$ & $O \forall$ & 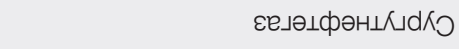 \\
\hline & yN & & IN & ૧ІфәняецЈ \\
\hline & $d 0$ & & $d 0$ & odıəบวKd \\
\hline & yN & & $\mathrm{dN}$ & ૧цфрәноОд \\
\hline & yN & & IN & 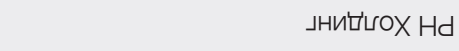 \\
\hline$\exists 09$ & $O \forall$ & $0 t$ & $O \forall$ & Y्र $\perp \forall G O H$ \\
\hline 91 & $O \forall$ & & $d 0$ & ГИОYКК \\
\hline & yN & & $\mathrm{d} N$ & વІфән wodபعe」 \\
\hline ○ 99 & $O \forall$ & ○ 乙9 & $O \forall$ & woduعe」 \\
\hline & $d 0$ & & dO & ৭ıфәнпाе \\
\hline & & & & 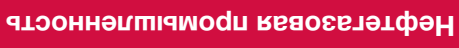 \\
\hline 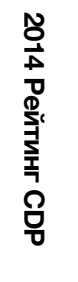 & 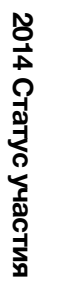 & 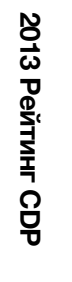 & 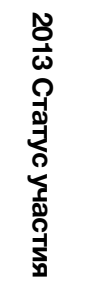 & иинечжох әинеяонәшиен \\
\hline
\end{tabular}

\begin{tabular}{|c|c|c|c|c|}
\hline 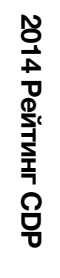 & 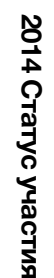 & 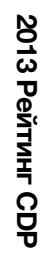 & 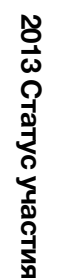 & иинешюох әинеяонәшиен \\
\hline
\end{tabular}




\section{$\exists$ 이 $\mathbf{v} 10$}

елницйәd чеяdəцни = \%

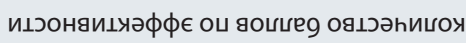

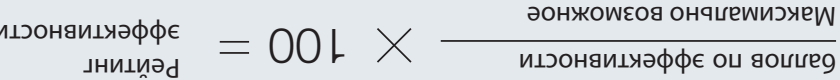
ояцวһицоо әоннәһКцоч

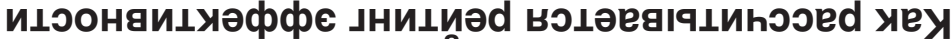

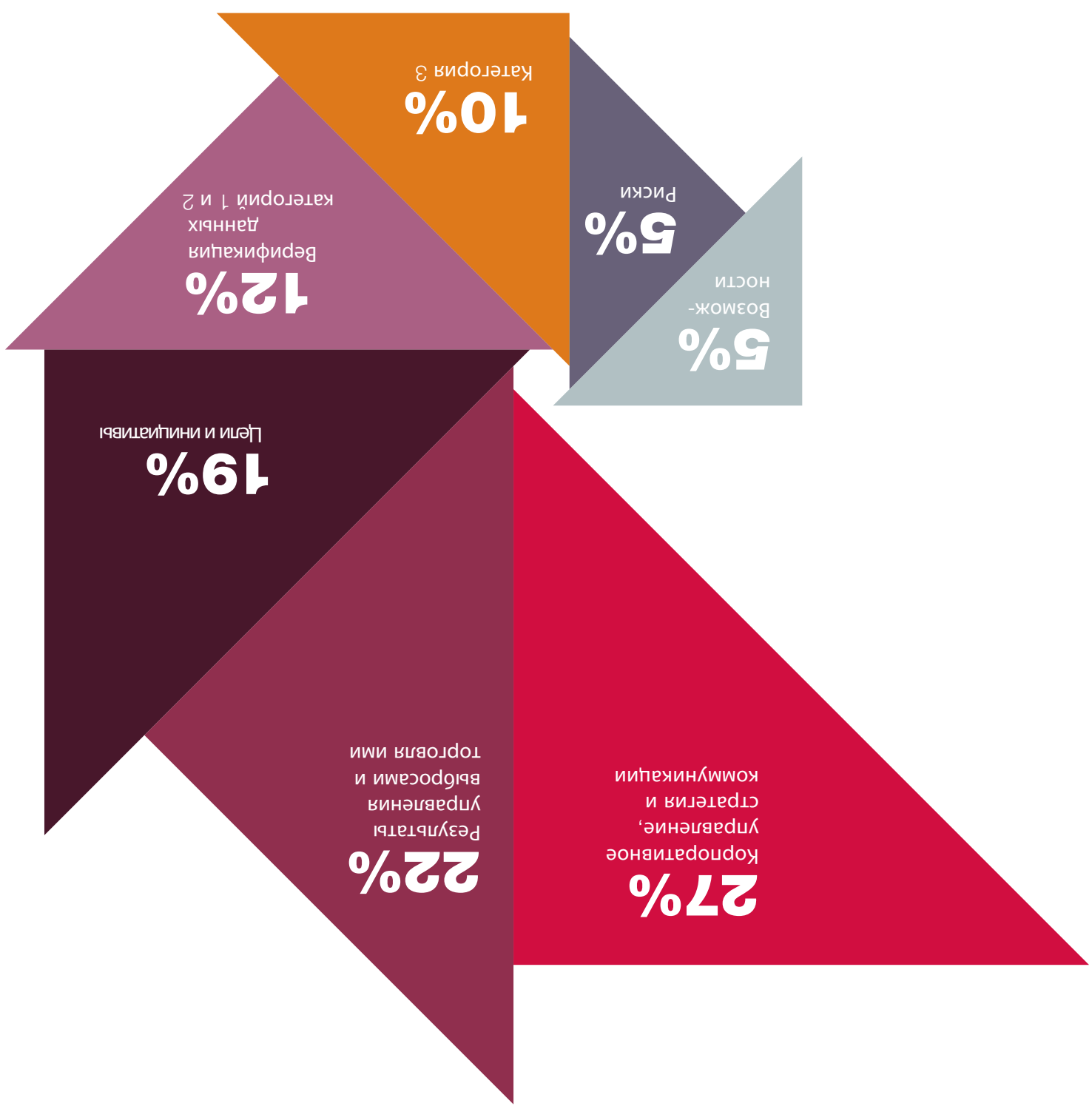

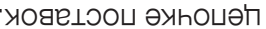

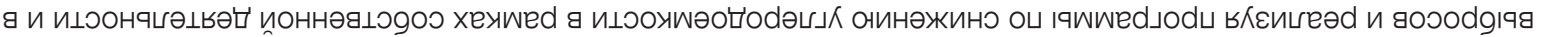

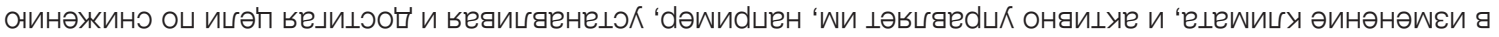

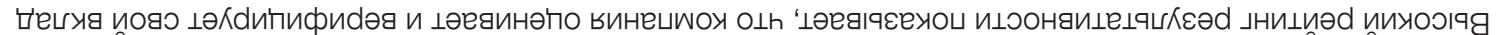

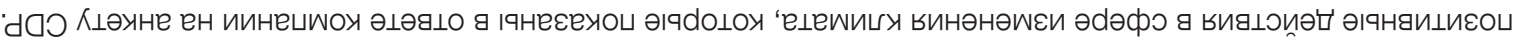

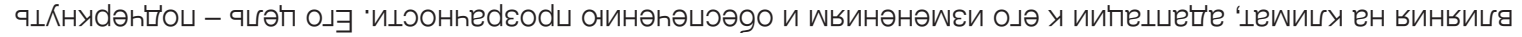

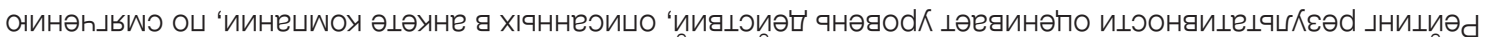

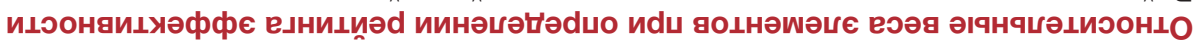




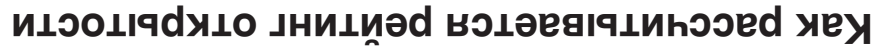

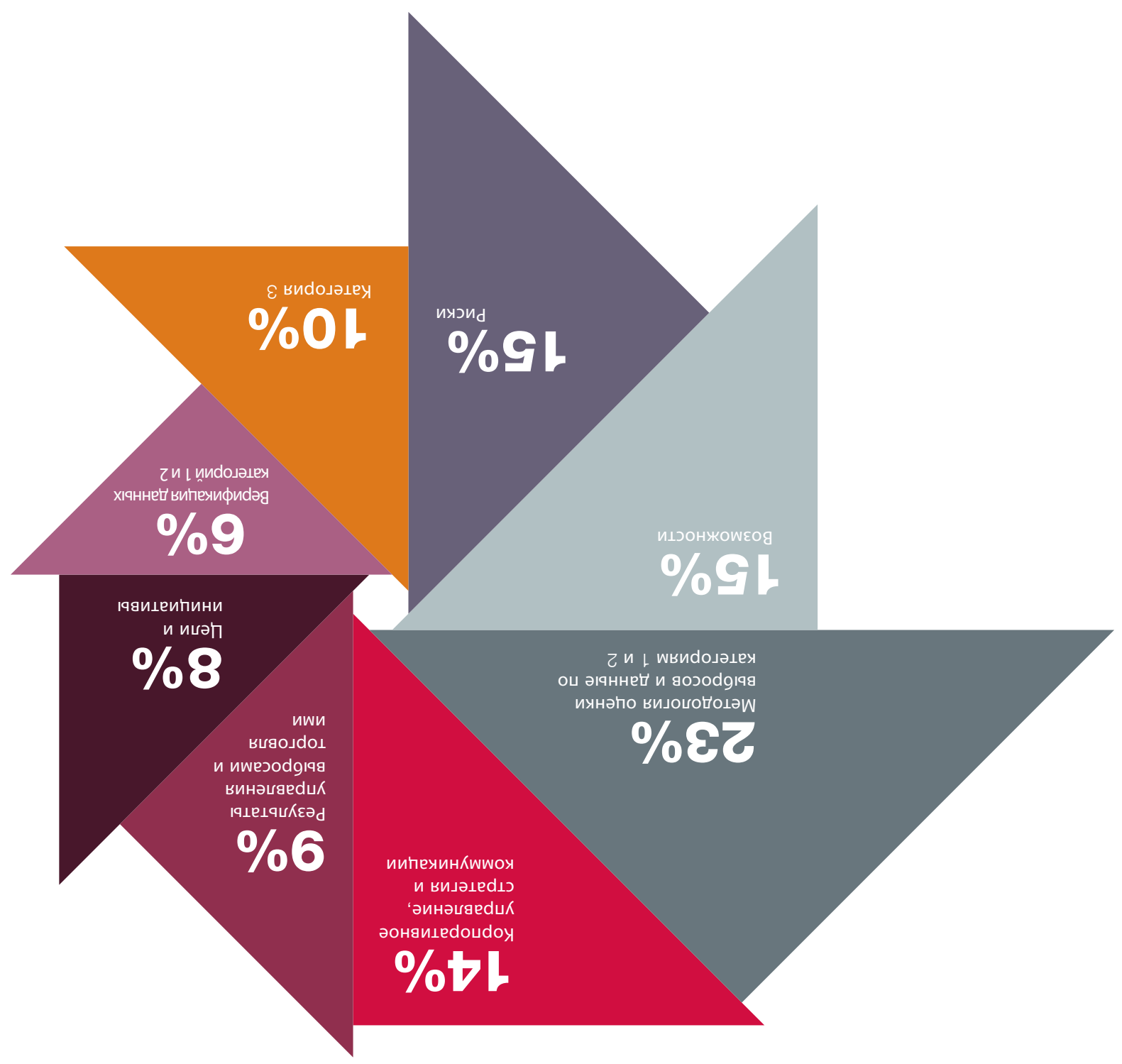

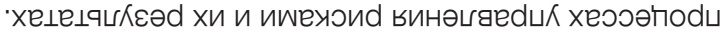

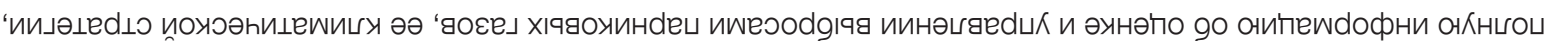

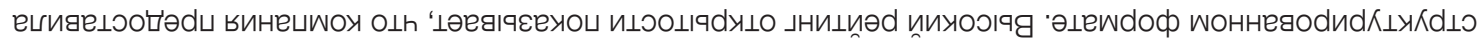

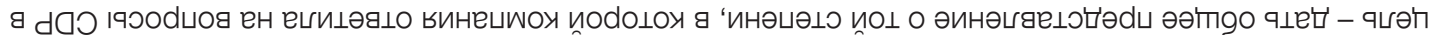

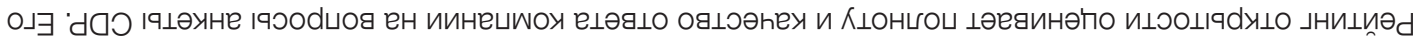

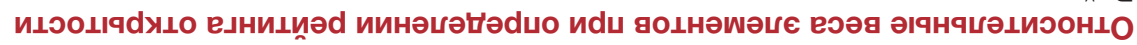


goce $\lrcorner$

хІчgоуинdе

goวodgıя

онинтпедуго

Оப иเәП

goke」

хІ9яочинdеப weวodgıя Oப १॰ว०Н $\perp \partial h \perp \mathrm{O}$

ИІООНЖОWЕОЯ и ихои

१॰วоняицүәффе и ецеพицу พәинәнәшєИ әинәцаеdபк

gotreg $n$

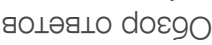
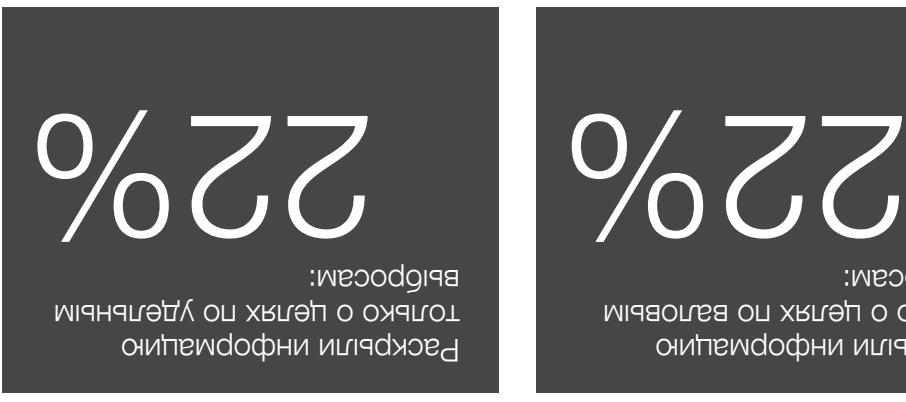

:weoodgıя

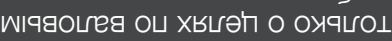

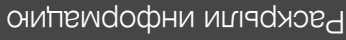

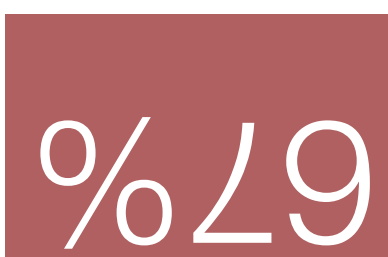

:ح ииdo」əıех्र хеวodgıя

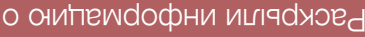

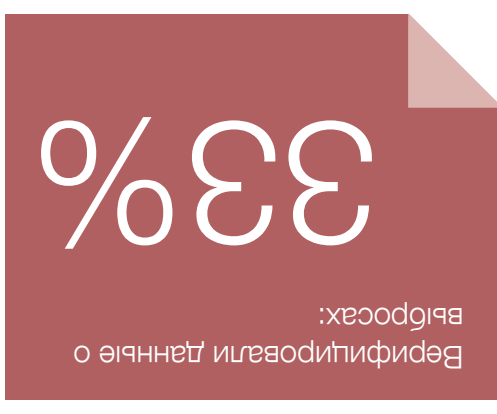

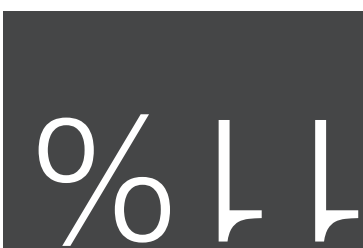

:weoodgıя

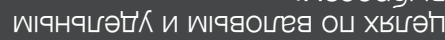

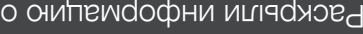

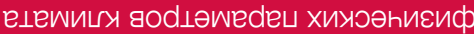
ижьинәнәшєи ว әІянневьяо 'ицонжошєоя

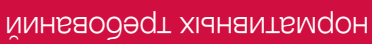

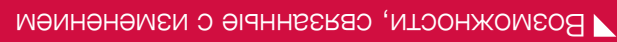
:ИเоОнжошвоя әІवняоноО
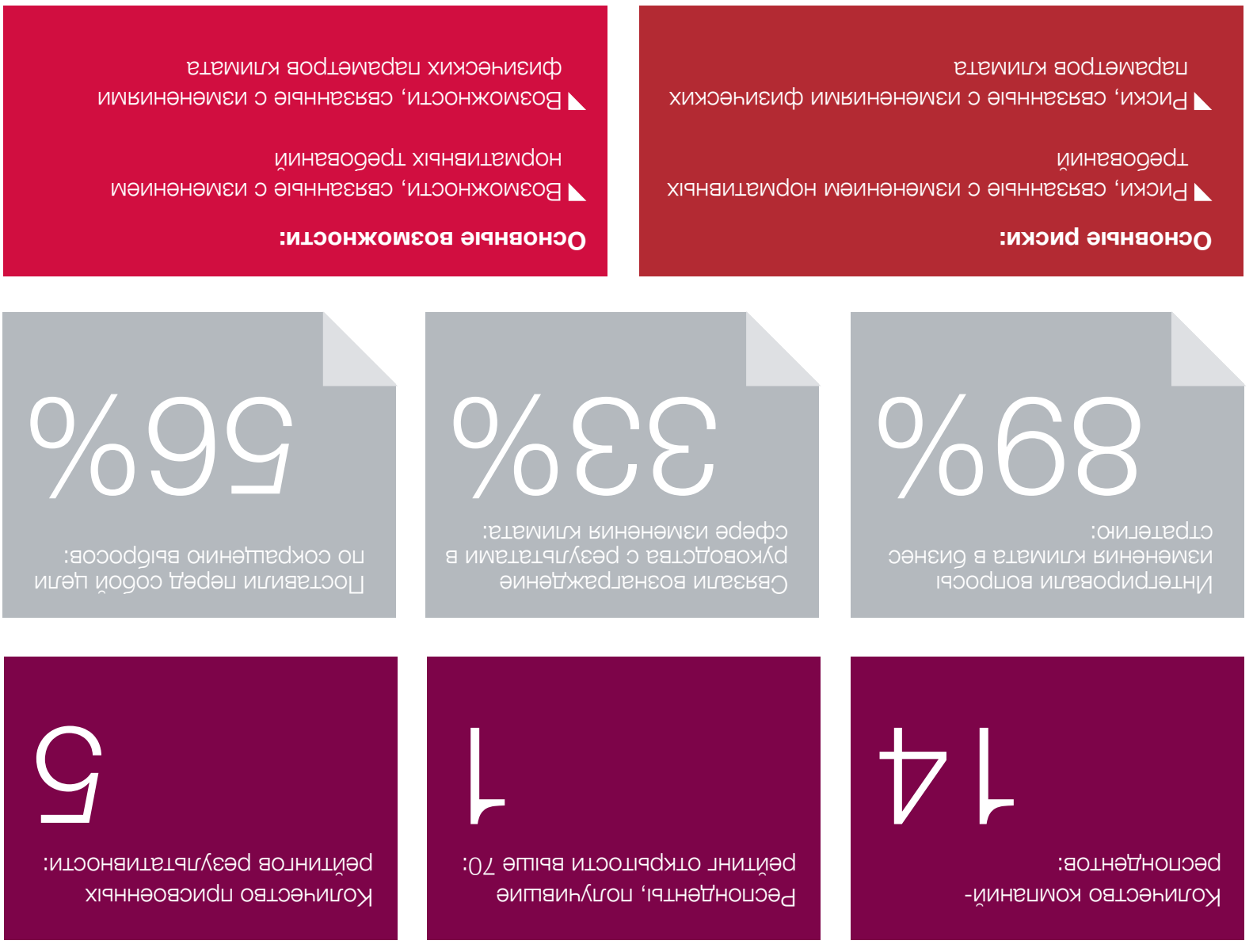


\begin{tabular}{|c|c|c|}
\hline 91 & - & ГИОУКК \\
\hline 乙乙 & - & «әинәЪжоdєоg» үинеg \\
\hline ¿乙 & ¿乙 & $\varepsilon е\lrcorner ә \perp \phi ә н \perp К\lrcorner$ dノ \\
\hline Ot & $L \nabla$ & ийгеуцгеd/ \\
\hline$\exists 09$ & Ot & $\mathrm{Y} \in \perp \forall G \mathrm{OH}$ \\
\hline$\exists 乙 9$ & 乙乙 & $\varepsilon \forall d \theta \exists$ \\
\hline$\exists \varepsilon 9$ & - & 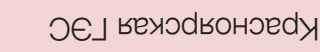 \\
\hline ○ 99 & ৩ २९ & woduعe」 \\
\hline$O \angle L$ & - & 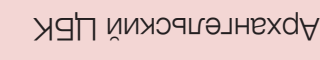 \\
\hline 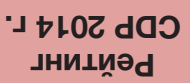 & 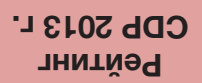 & иинешжох्र \\
\hline
\end{tabular}




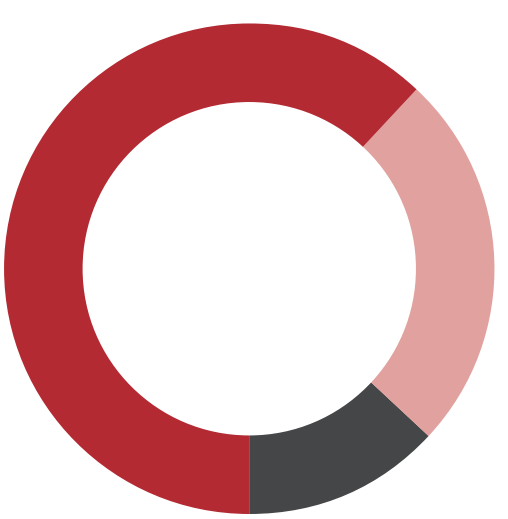

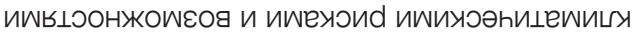

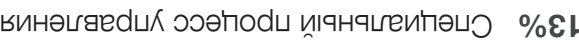

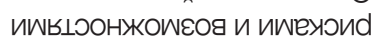

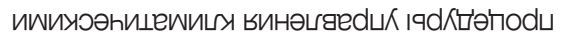

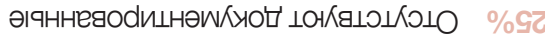

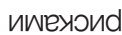

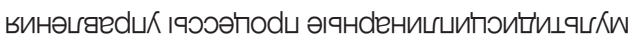

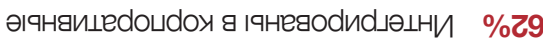

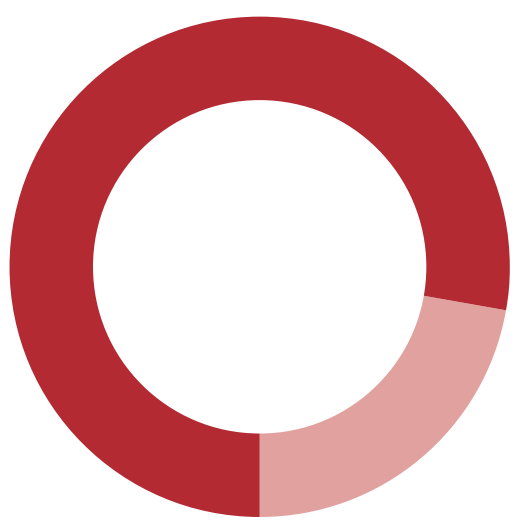

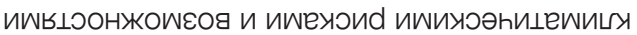

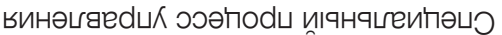

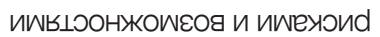

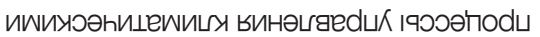

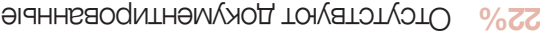
иweyond

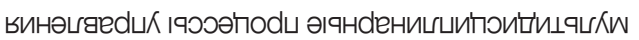

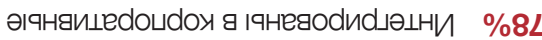

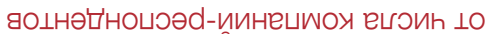

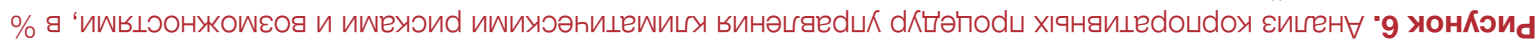

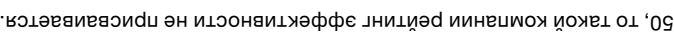

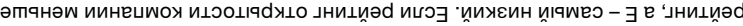

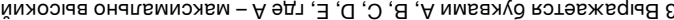
'wosodues o ии

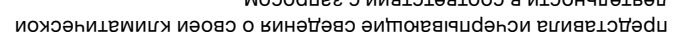

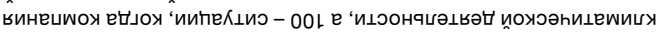

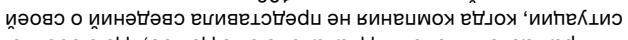

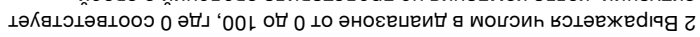

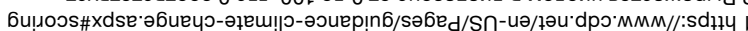

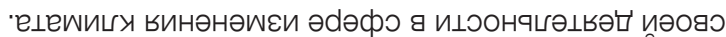

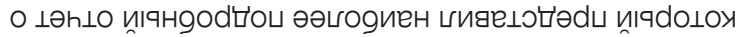

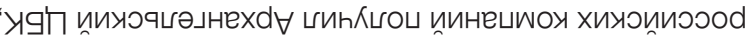

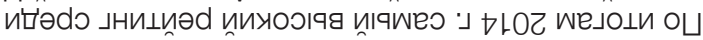

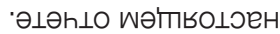

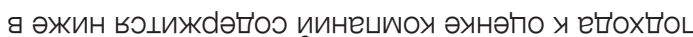

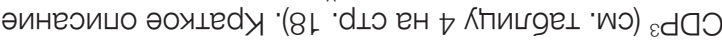

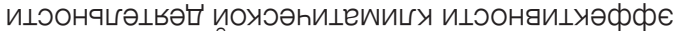

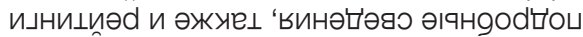

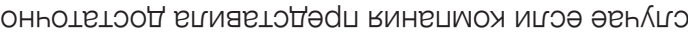

a e ' $2 d 0 \supset$ И І

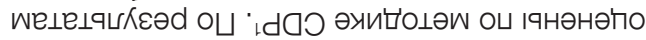

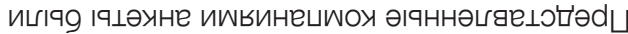

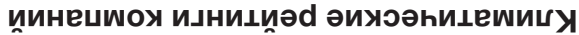

'иıеed

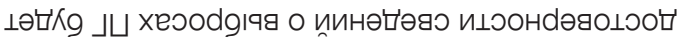

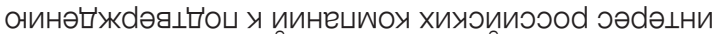

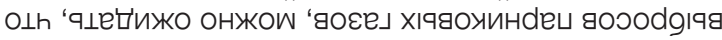

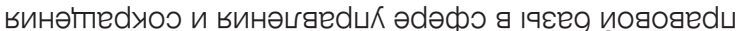

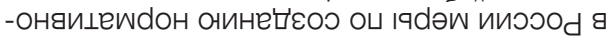

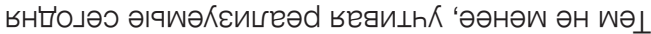

$\cdot \partial \perp \partial h \perp 0$

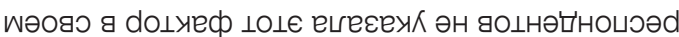

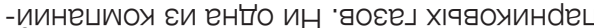

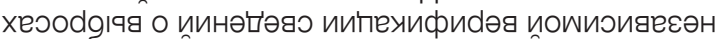

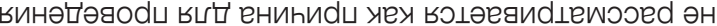

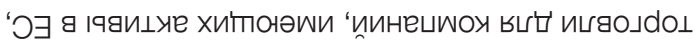

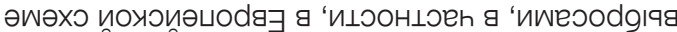

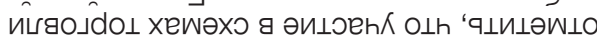

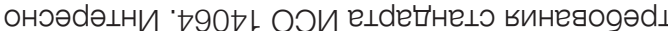

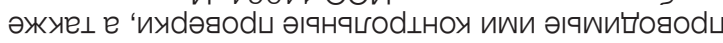

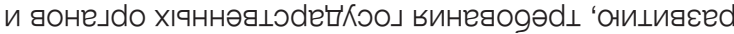

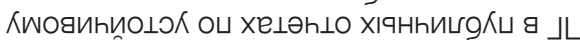

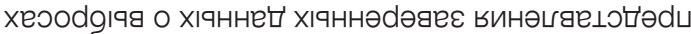

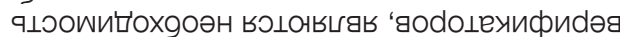

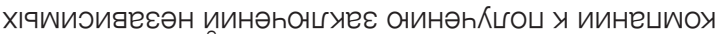

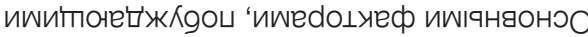

•Ш xeoodgiag

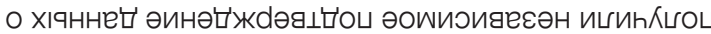

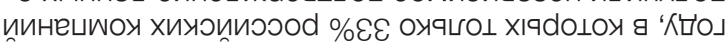

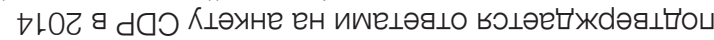

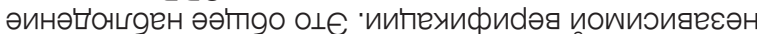

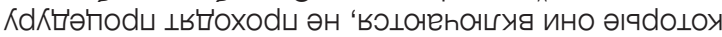

घ '

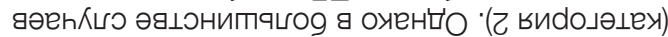

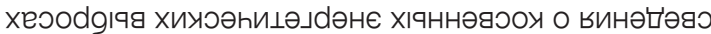

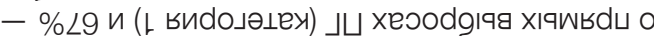

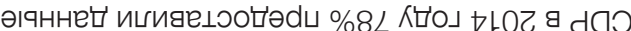

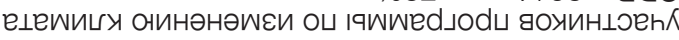

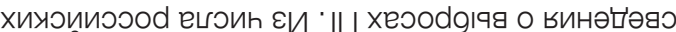

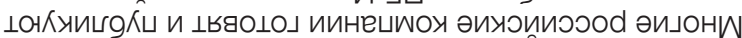

JU xeoodgiqg

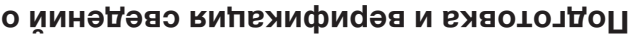




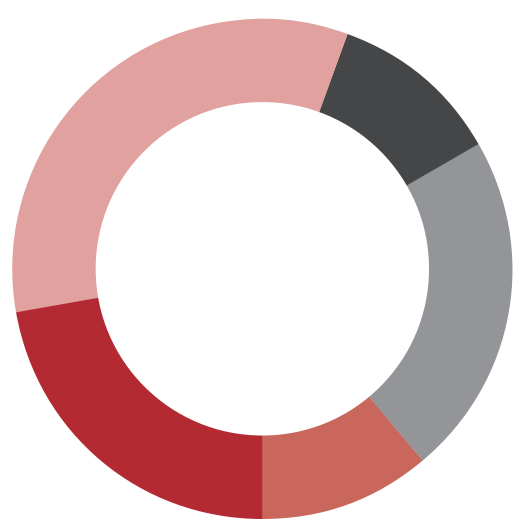

оневехк ән $\% 0$ ।१'๋ədo

иә'томежК

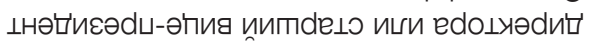

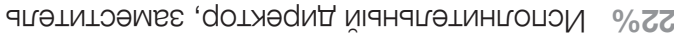

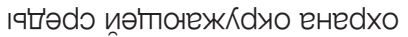

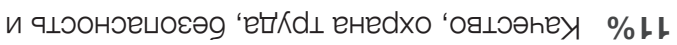

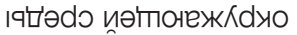

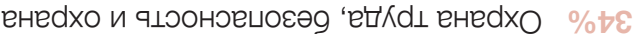

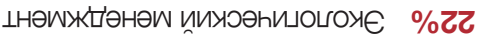

†LOZ

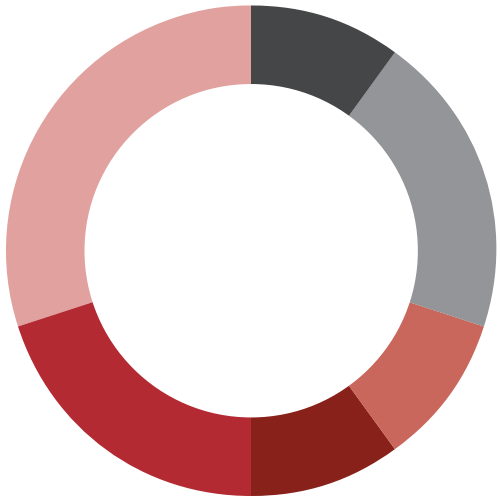

Оневехк әН \%OL

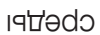

иәтпоеж久

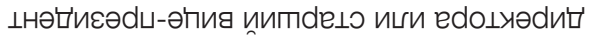

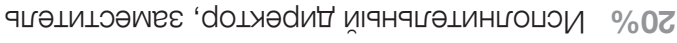

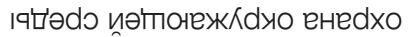

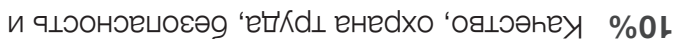

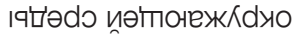

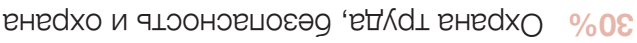

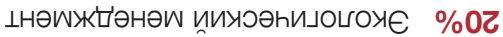

ELOZ

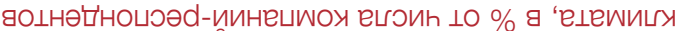

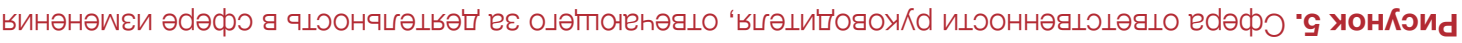

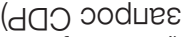

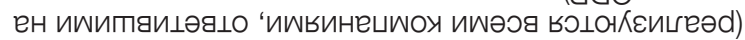

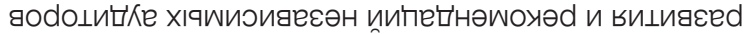

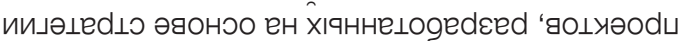

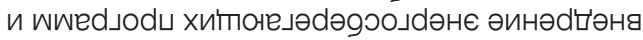

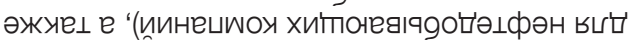

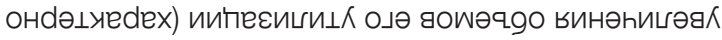

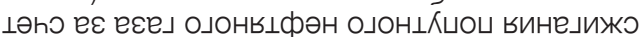

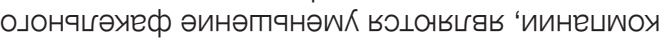

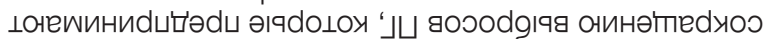

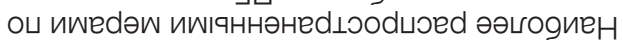

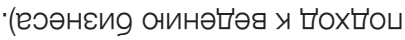

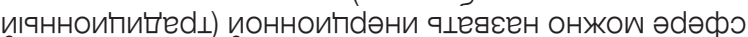

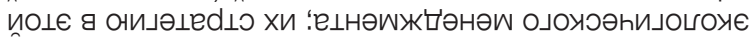

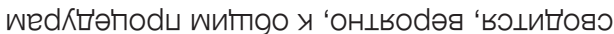

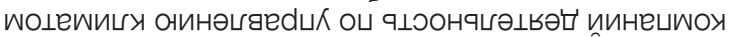

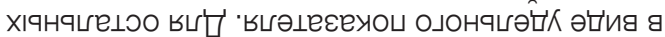

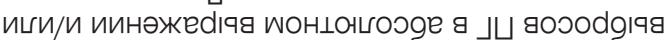

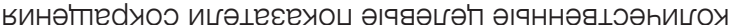

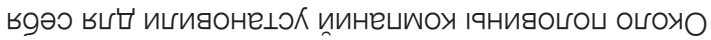

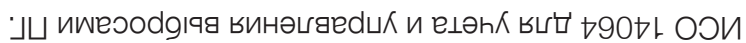

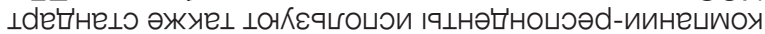

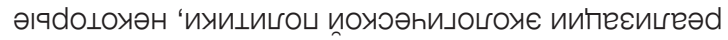

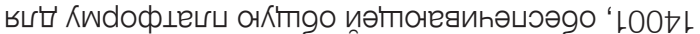

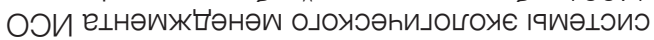

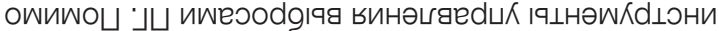

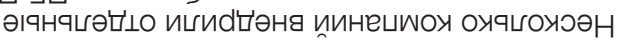

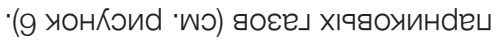

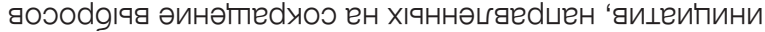

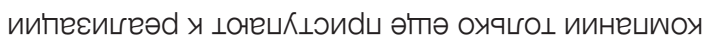

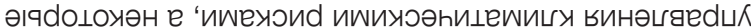

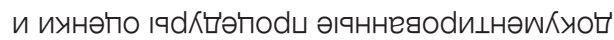

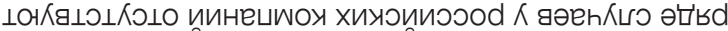

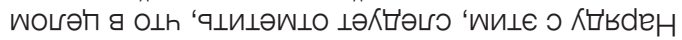

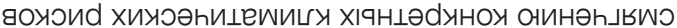

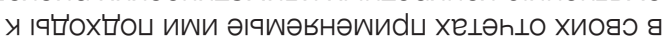

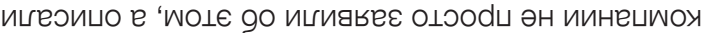

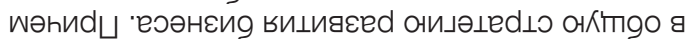
І

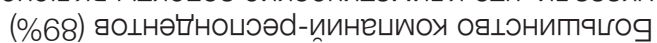
'ецешиц् иинәнәшєи

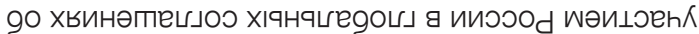

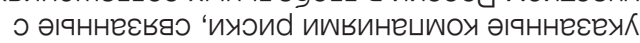
и и

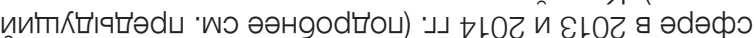

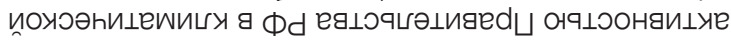
'онषияәһ० '।

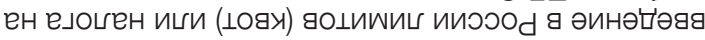

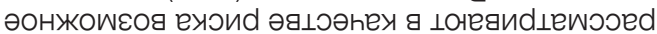

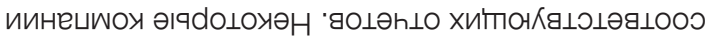

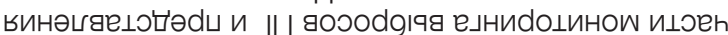

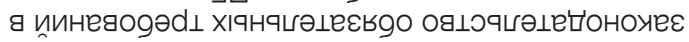

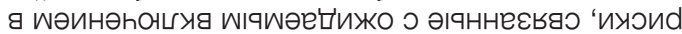

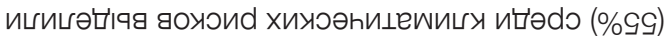

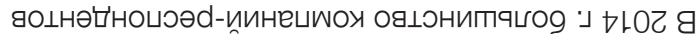

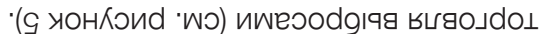

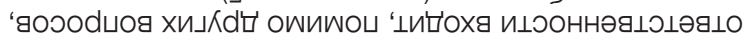

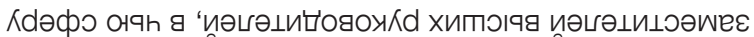

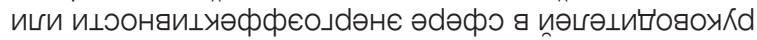

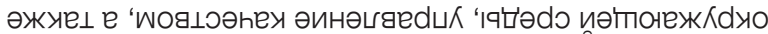




\begin{tabular}{|c|c|c|c|c|}
\hline+ & + & 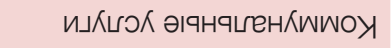 & виээо d $\mathrm{HO}^{\circ} \mathrm{\epsilon}$ & \multirow{5}{*}{$* * \forall S$} \\
\hline+ & + & 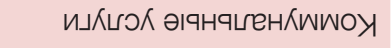 & G-У્ર」О ЬヨН€ & \\
\hline+ & + & 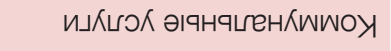 & W/LdO $\Phi$ & \\
\hline+ & + & вилdәнє & 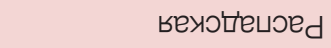 & \\
\hline+ & + & 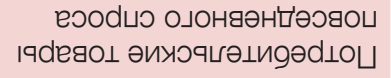 & ннеப'-qเьия-พพиg & \\
\hline+ & + & ІагеиdәцеW & ииицеучге & \multirow{13}{*}{$* O \forall$} \\
\hline- & + & 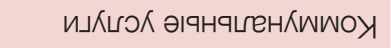 & $\llcorner-Y \mid \downarrow\lrcorner \perp$ & \\
\hline+ & + & вилdән€ & 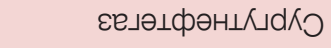 & \\
\hline- & + & ıчгеиdəıеW & " $\forall O$ O & \\
\hline+ & + & вилdәнє & $Y \in \in \perp \forall G O H$ & \\
\hline- & + & әинәжgенэоdцуәцє & YOEOW & \\
\hline+ & - & вилdәнЄ & ГИОУКЦ & \\
\hline+ & + & 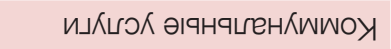 & Ј」 ьехэдьонэеdУ्र & \\
\hline+ & + & еуицә」dәнє & woduعe」 & \\
\hline+ & + & ІаьеиdəцеW & $\varepsilon \forall d g \exists$ & \\
\hline+ & + & І૧ОНенИФ & 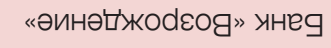 & \\
\hline+ & - & І9เеиdəıеW & 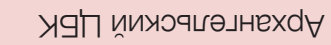 & \\
\hline$\cdot\lrcorner \bullet\llcorner O Z$ & 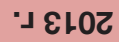 & Һоцхәว & иинешжох әинеяєен & \\
\hline
\end{tabular}

·」 †LОZ и \&L0Z в ецемиц्र

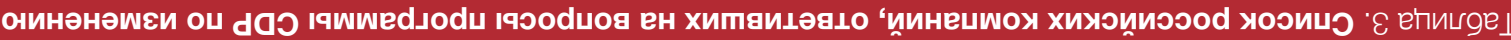

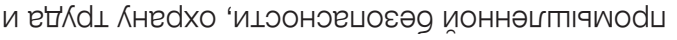

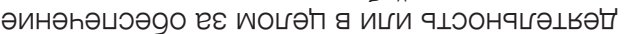

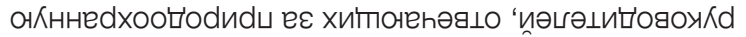

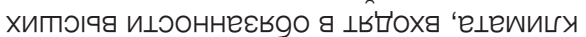

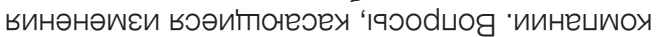

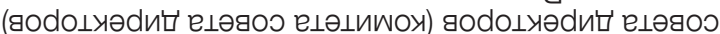

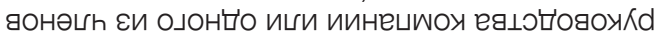

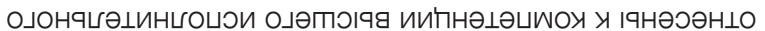

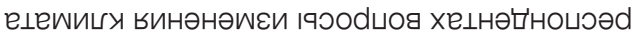

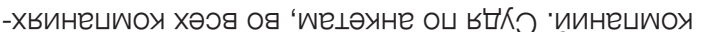

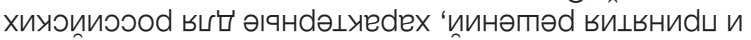

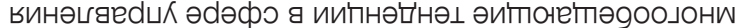

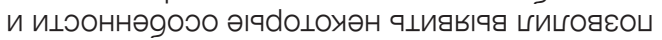

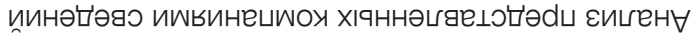

ецешиц мәинәнәшєи э хІवнневья

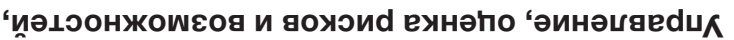

'иинечพоу $\Lambda\lrcorner \Lambda \mathrm{d}$

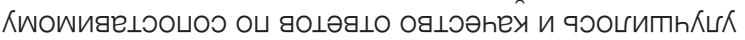

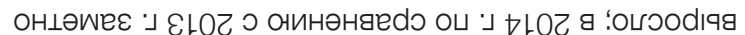

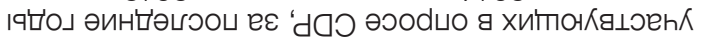

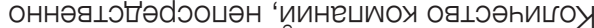

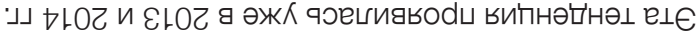

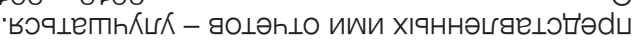

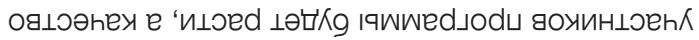

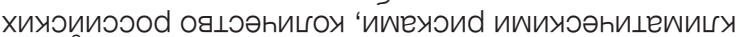

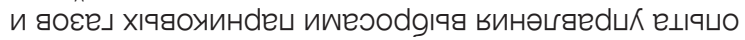

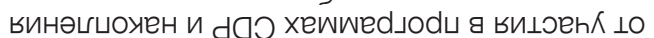

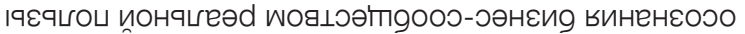

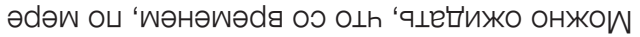

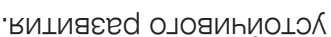

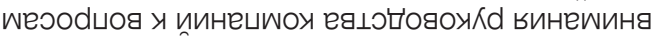

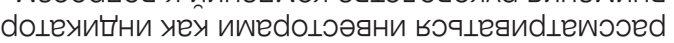

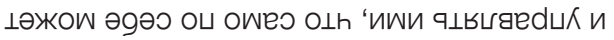

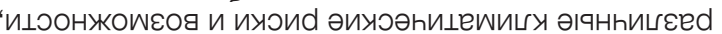

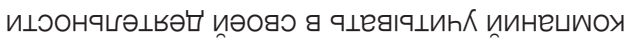

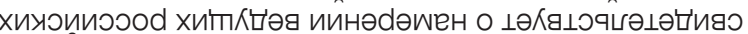

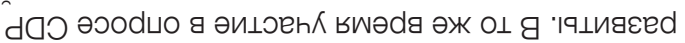

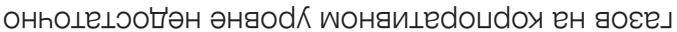

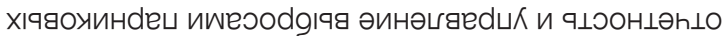

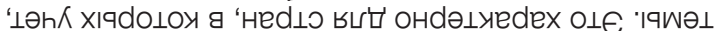

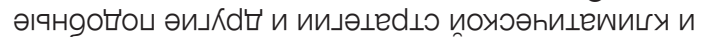

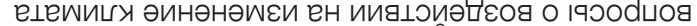




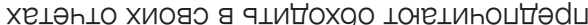

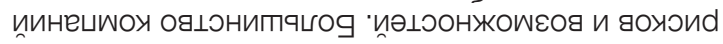
хи'поКя

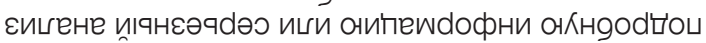

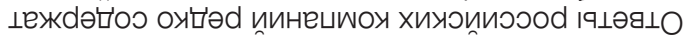

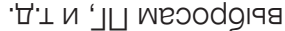

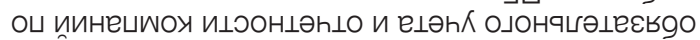

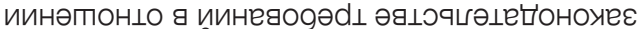

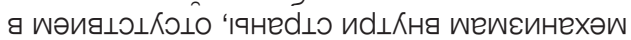

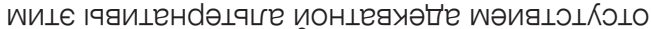

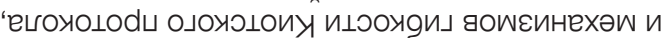

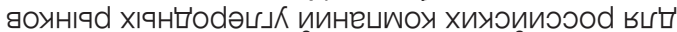
잉

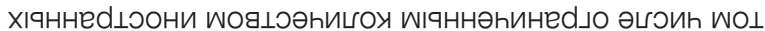

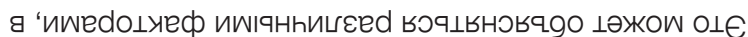

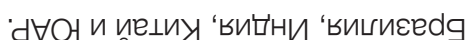

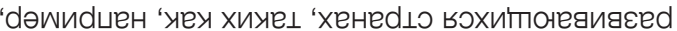

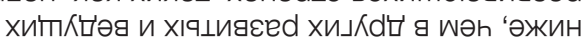

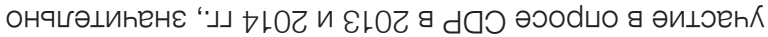

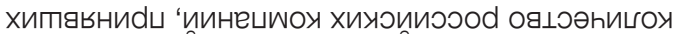

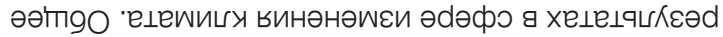

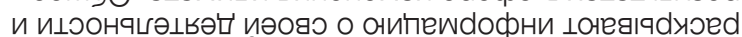

и dQつ әwwed $\lrcorner$ od

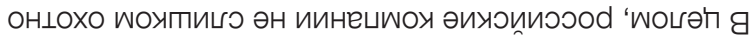

I9பоовІ9я әІяняоноО
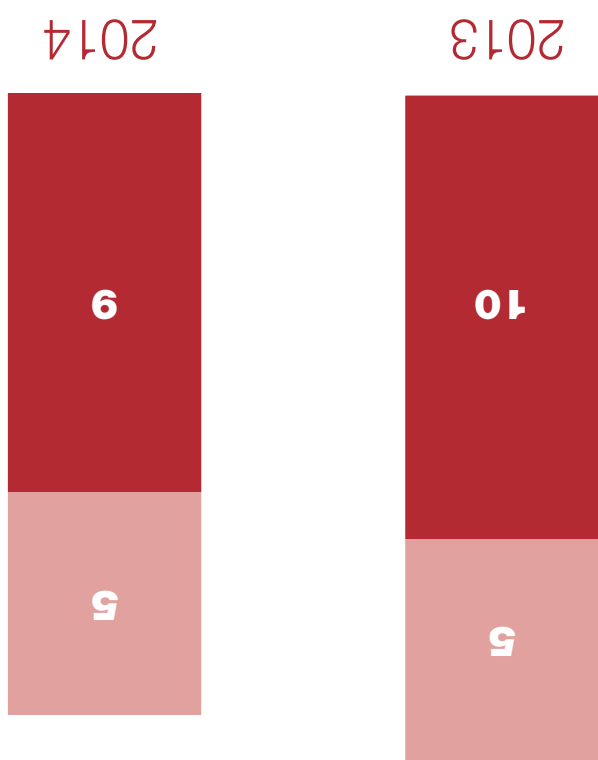

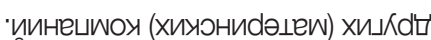

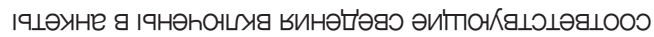

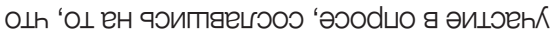

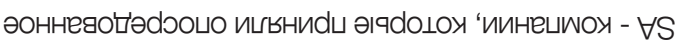

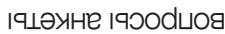

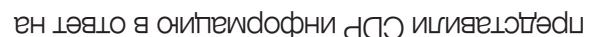

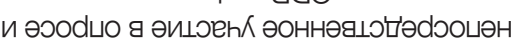

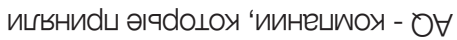

$(\varepsilon-$ оннеяоџәdэочо

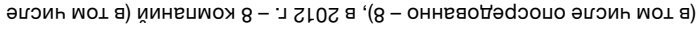

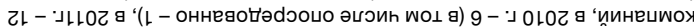

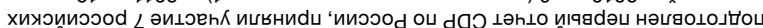

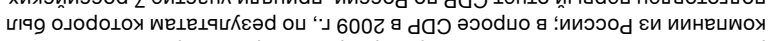

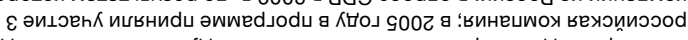

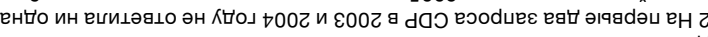
ииээод в ацооначәцьә

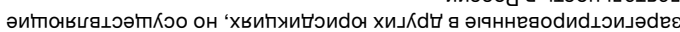

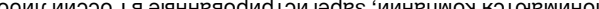

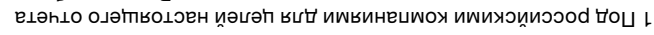

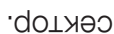

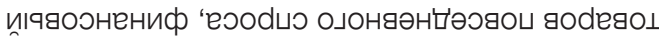

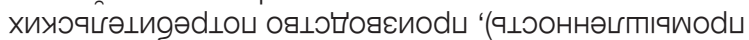

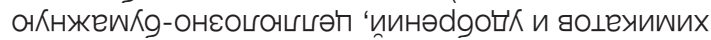

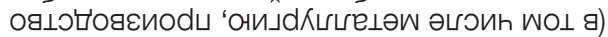

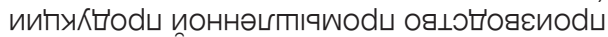

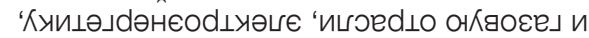

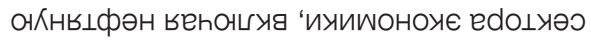

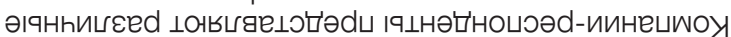

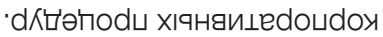

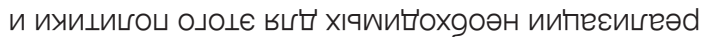

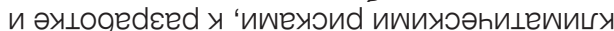

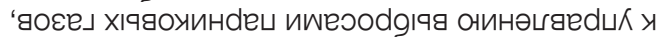

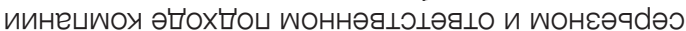

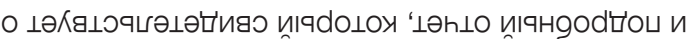

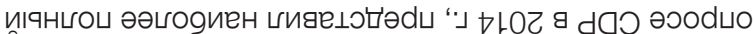

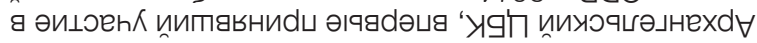

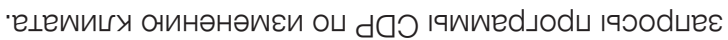

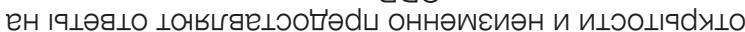

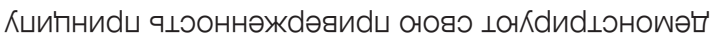

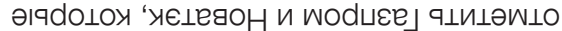

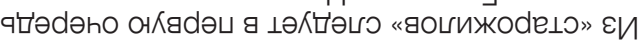

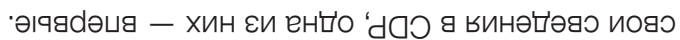

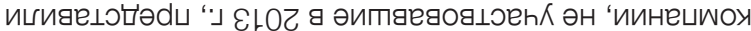

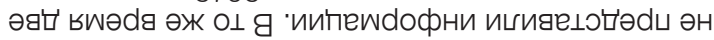

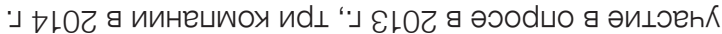

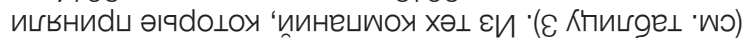

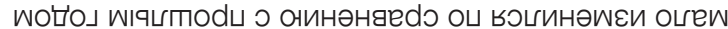
• ¿ڤИ०००d

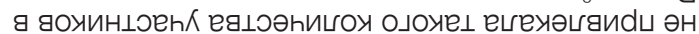

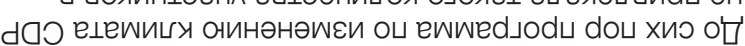

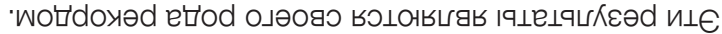

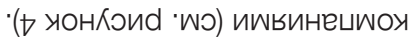

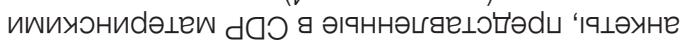

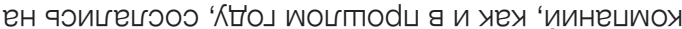

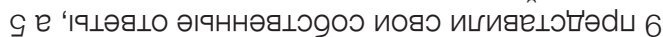

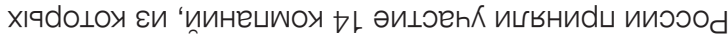

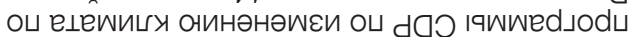

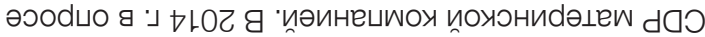

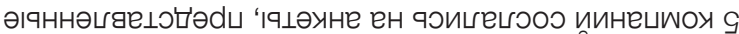

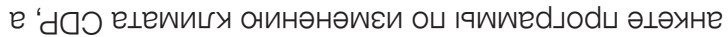

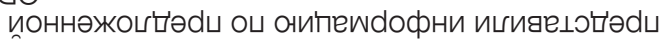

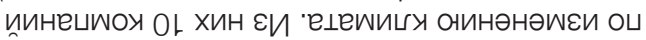

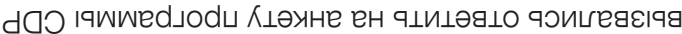

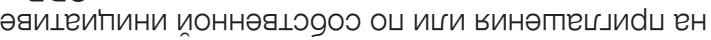

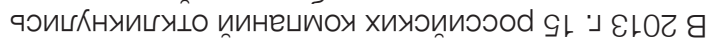

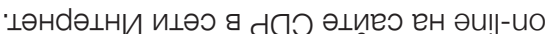

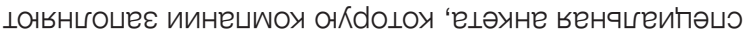

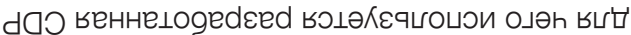

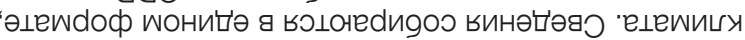

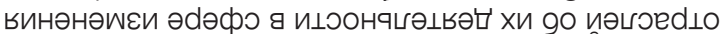

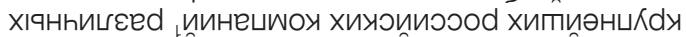

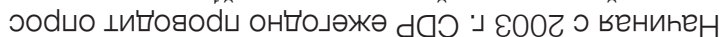

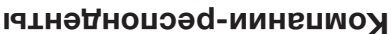


·ədəфо иохоәһицеพицу

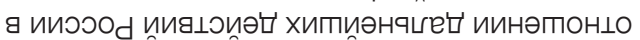

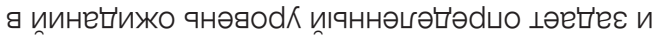

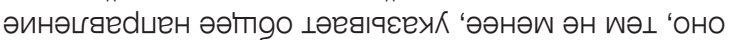

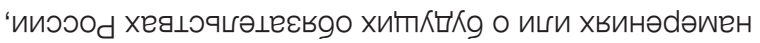

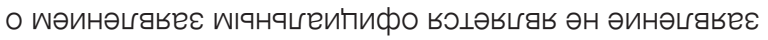

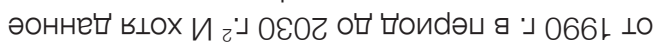

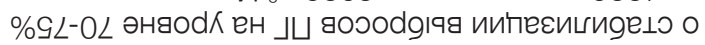
'wКพиник чехеу 'иц'ப'

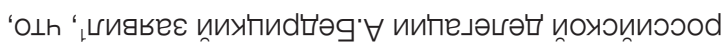

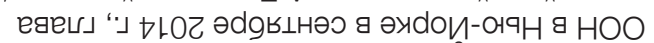

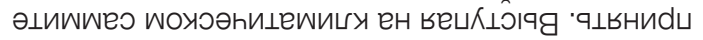

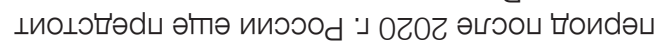

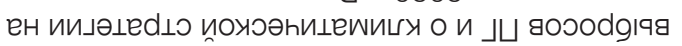
оинәжино оч ХธьәП о әинәпәd әонवцәцеһнохО

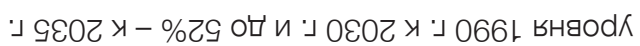

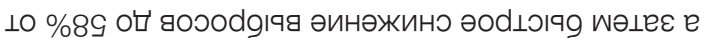

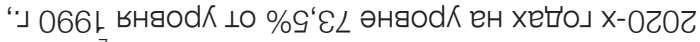

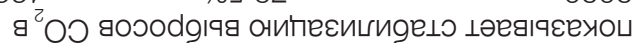

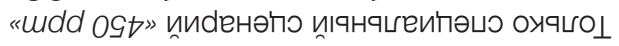

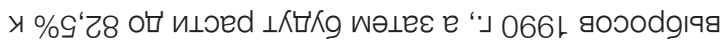

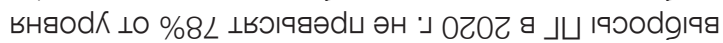

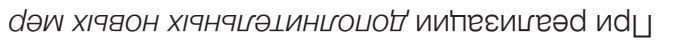

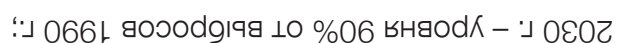

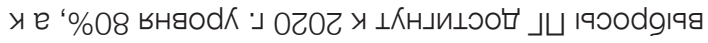

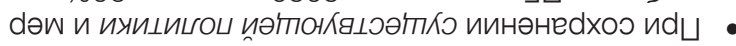

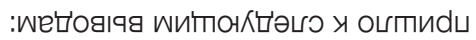

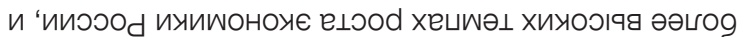

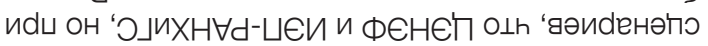

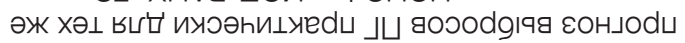

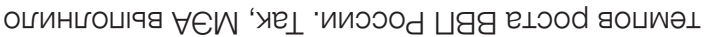

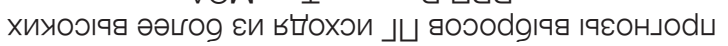




\section{OSOZ GtOZ OtOZ GEOZ OEOZ GZOZ OZOZ GLOZ OLOZ GOOZ}

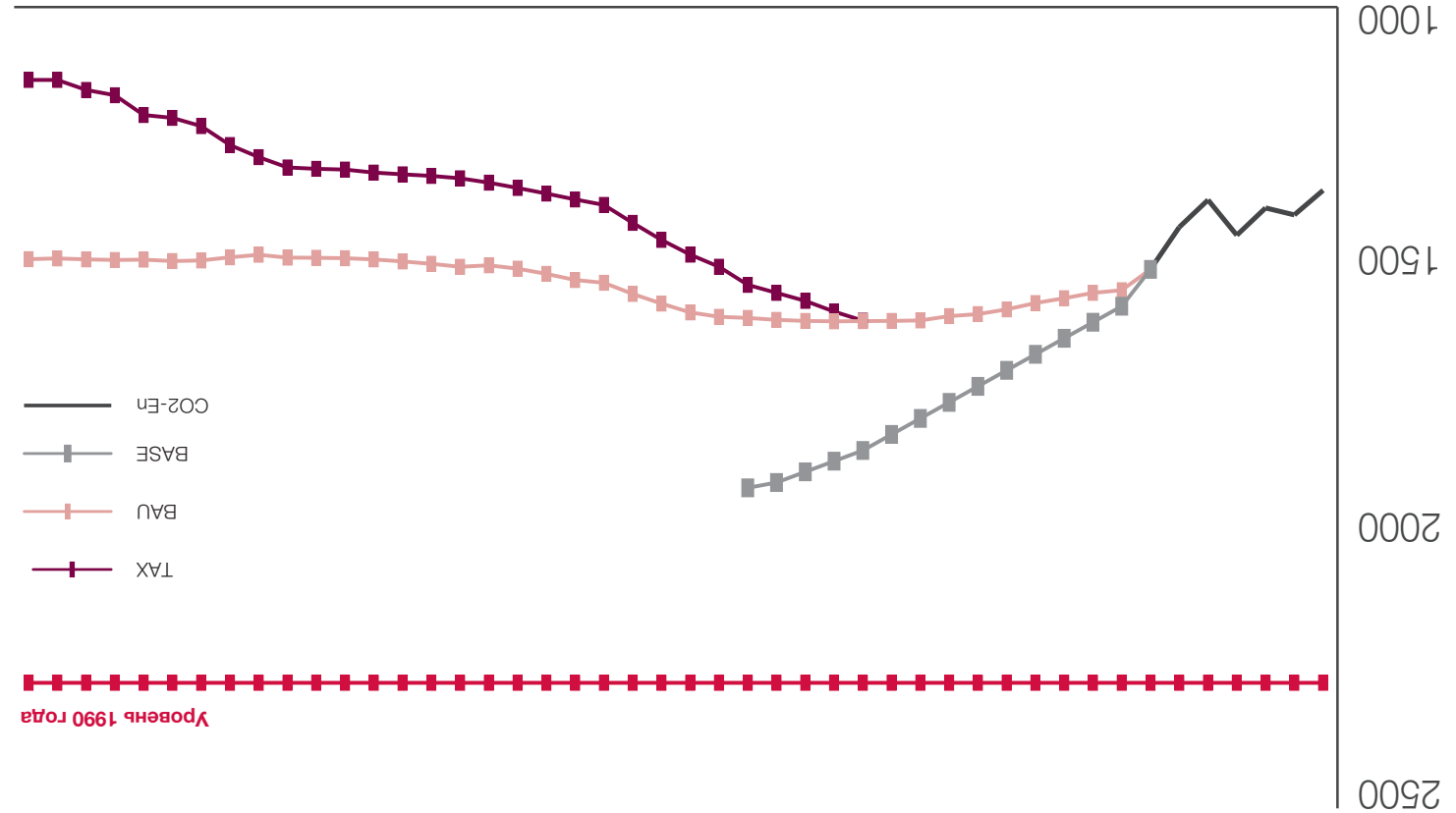

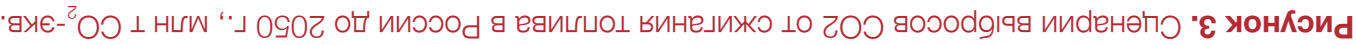

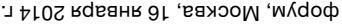

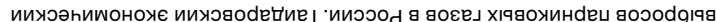

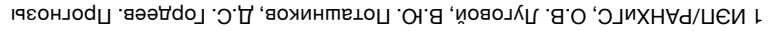

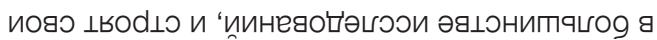

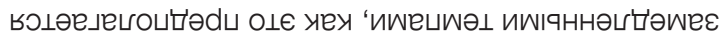

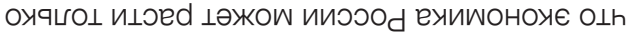

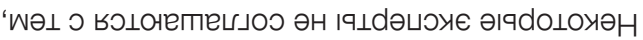

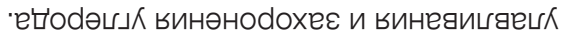

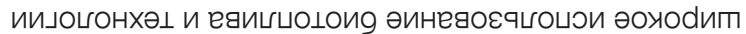

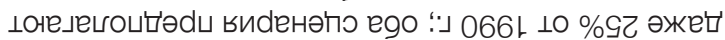

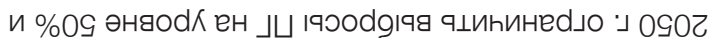

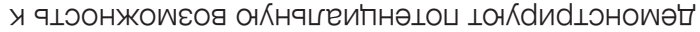

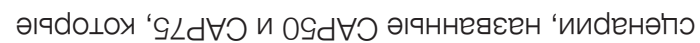

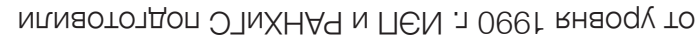

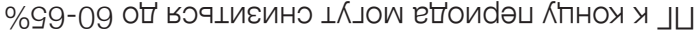

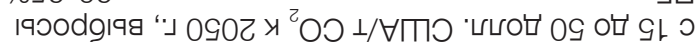

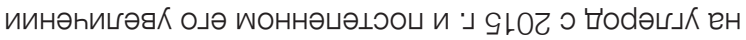

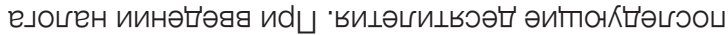

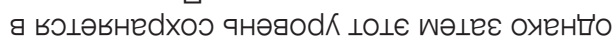

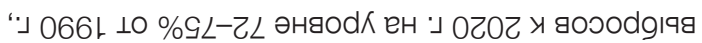

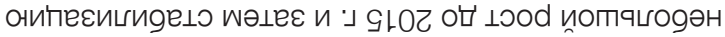

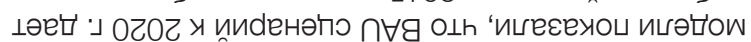

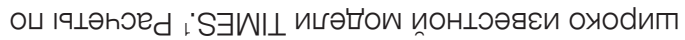

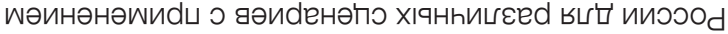

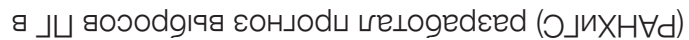

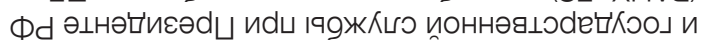

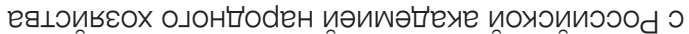

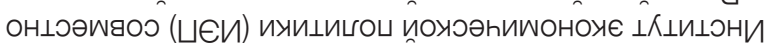

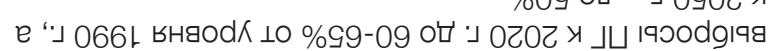

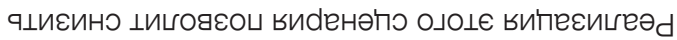

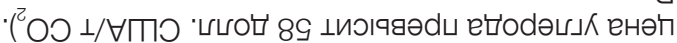

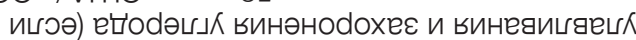
ии

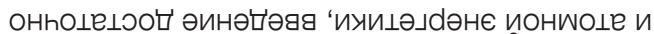

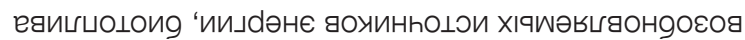

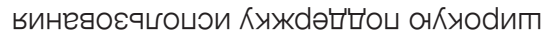

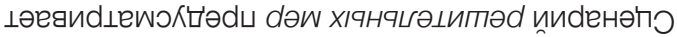

•əнаодर

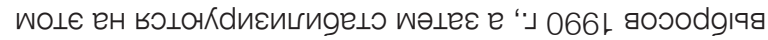

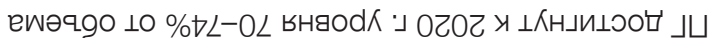

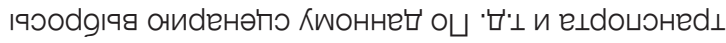

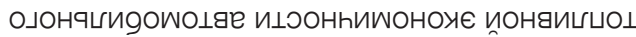

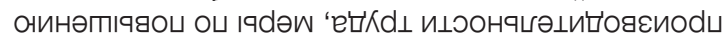

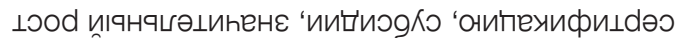

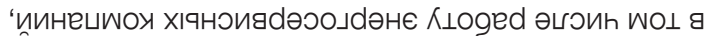

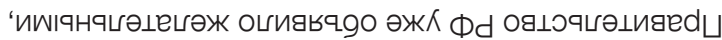

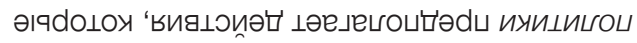

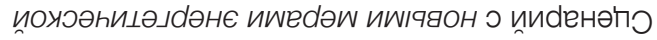
'ப'॰ а \%

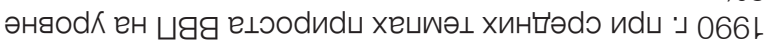

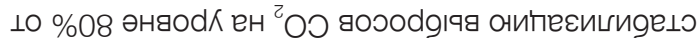

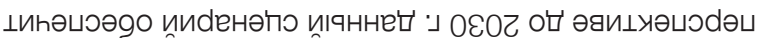

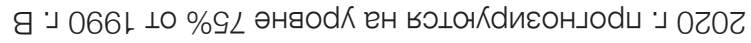

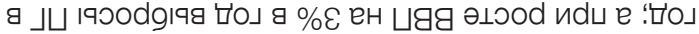

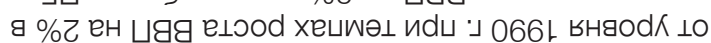




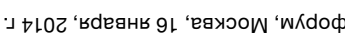

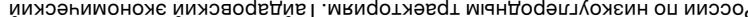

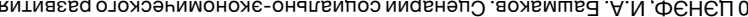
'

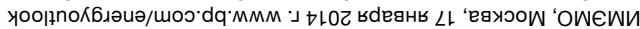

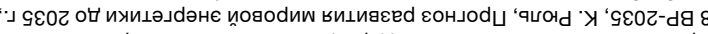

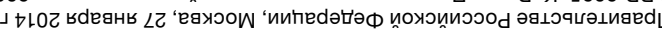

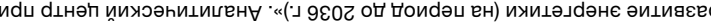

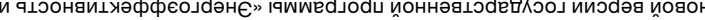
виће

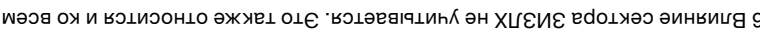

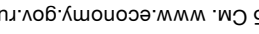

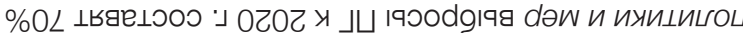

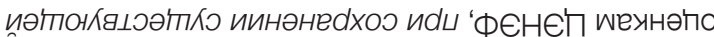

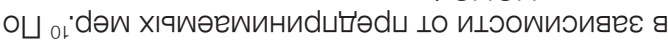

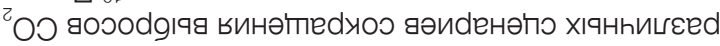

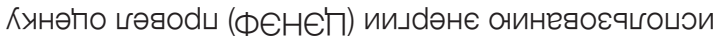

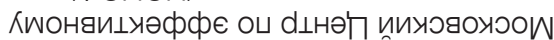

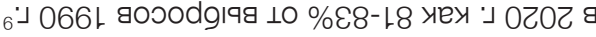

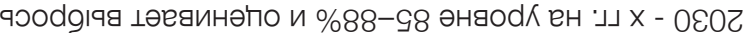

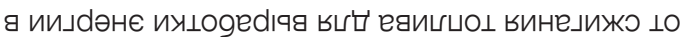

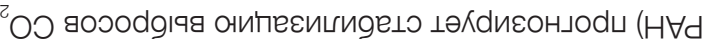

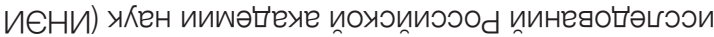

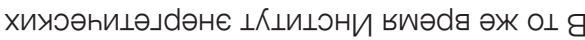

\% $\%$ SL-EL - 」 $0 Z 0 乙$

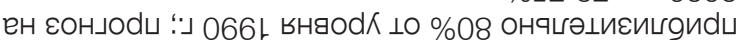

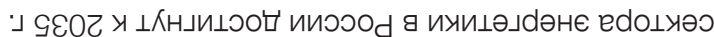

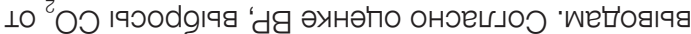

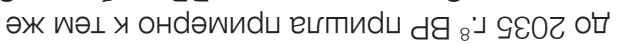

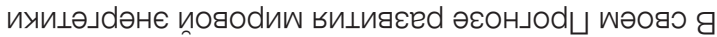

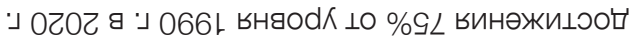

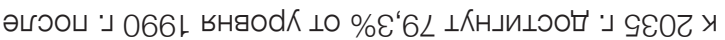

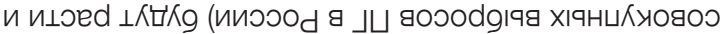

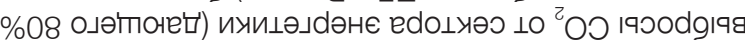

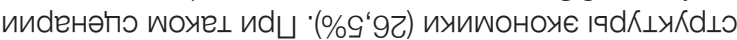

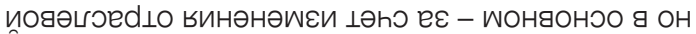

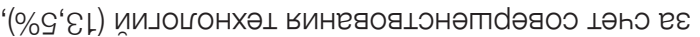

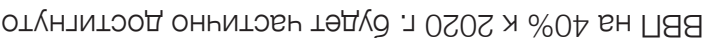

ицวохพәо」dәнє әинәтпе

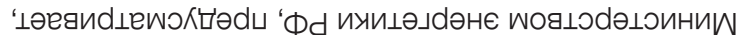

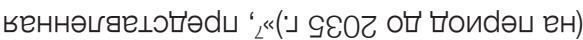

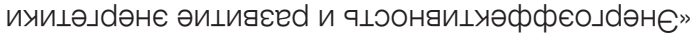

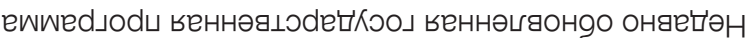

$$
\text { 9'иицdәне }
$$

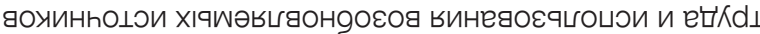

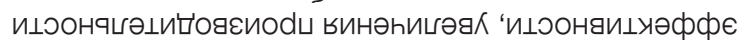

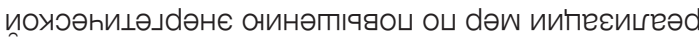

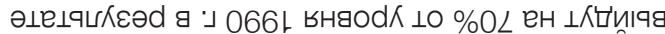

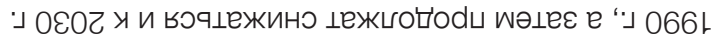

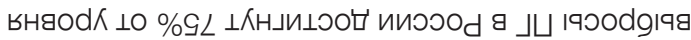

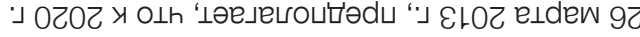

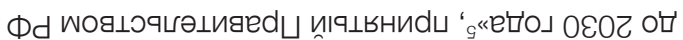

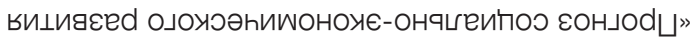

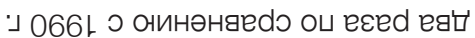

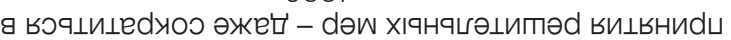

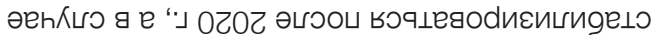

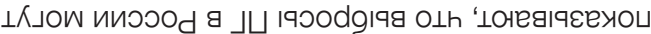

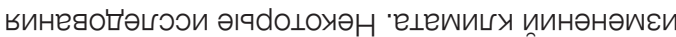

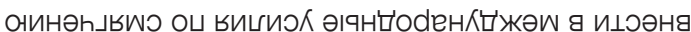

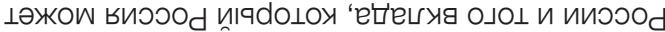

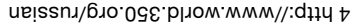
n':uolunojasn':MMM//:dz74 \&

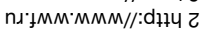

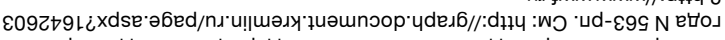

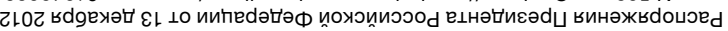

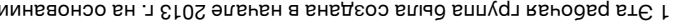

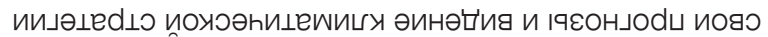

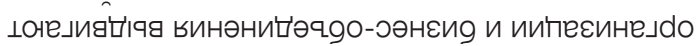

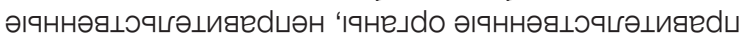

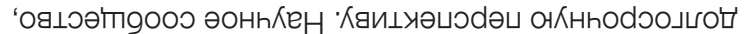

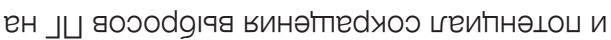

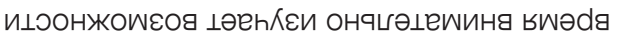
әәтььоцоен а виоэод "」

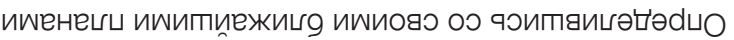

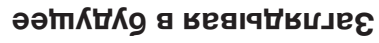

'ецешицу винәнәшєи wәเgodப

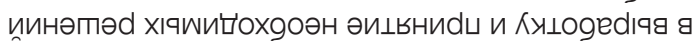

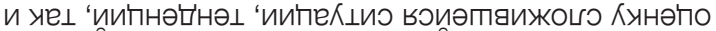

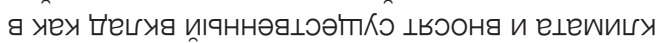

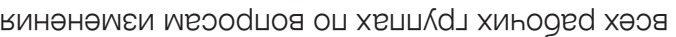

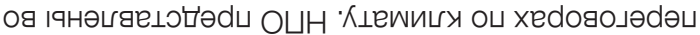

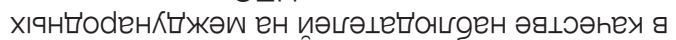

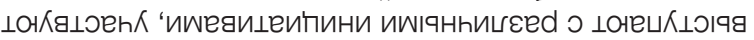

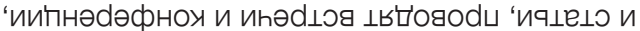

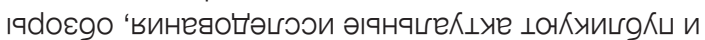

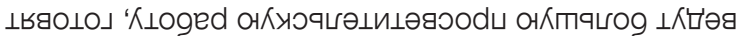

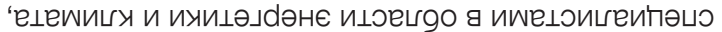

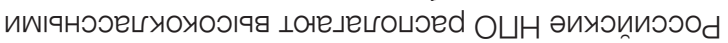

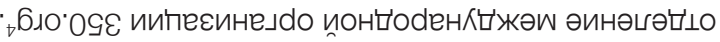

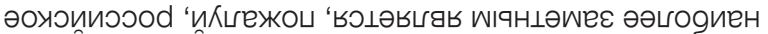

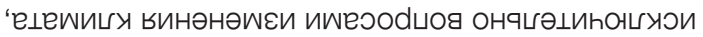

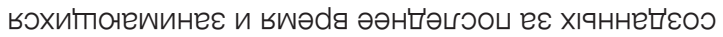

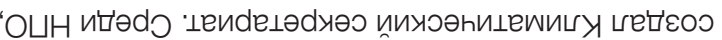

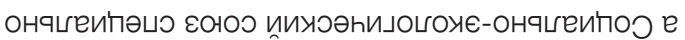

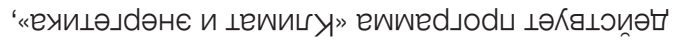

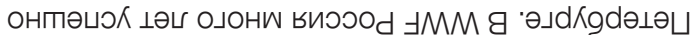

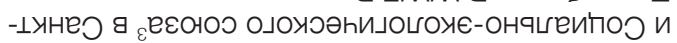

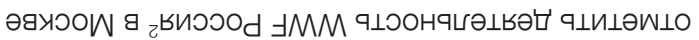

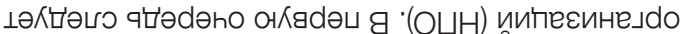

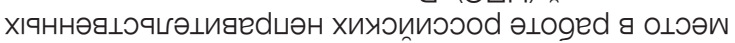
әон'பи

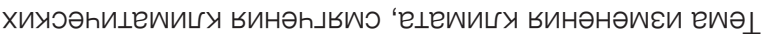

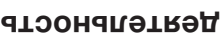
ьеннәяцәәтाо и ецешичх әинәнәшєИ

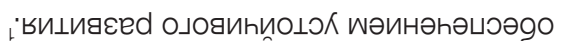

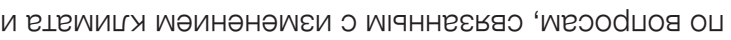

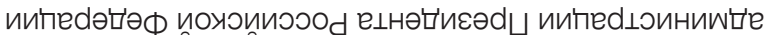

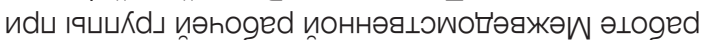

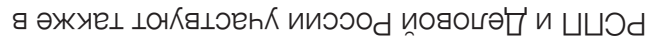

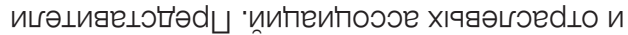

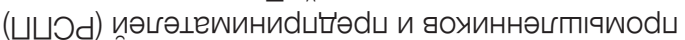

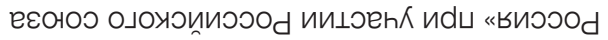

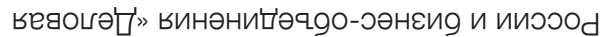

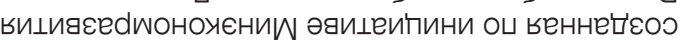

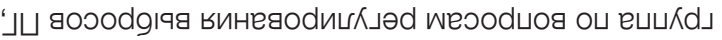

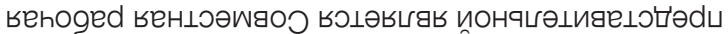

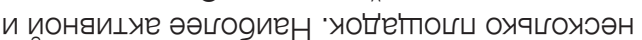

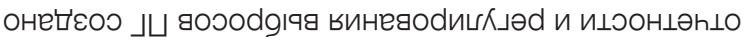

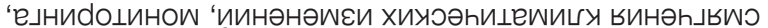

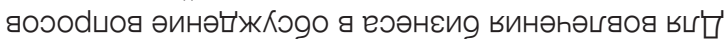




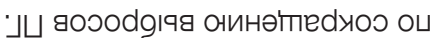

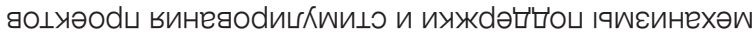

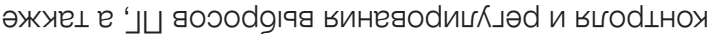

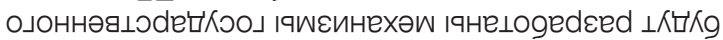

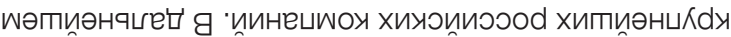

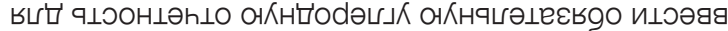

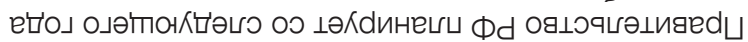

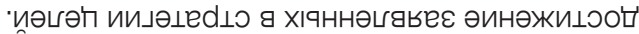

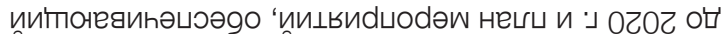

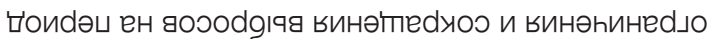

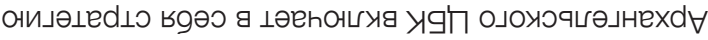
\lrcorner$\sqcup$ иพе

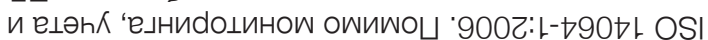

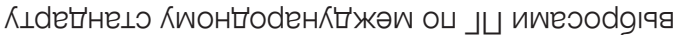

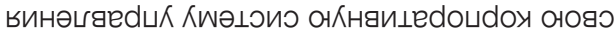

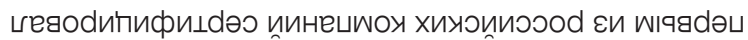

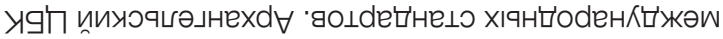

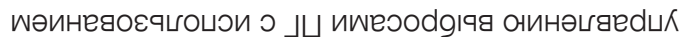

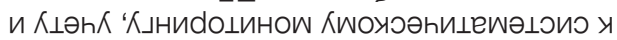

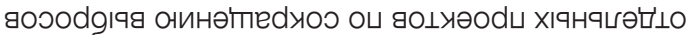

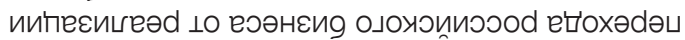

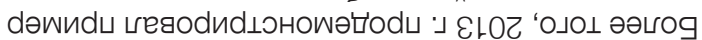
·」 घoวodgıя винә'пе

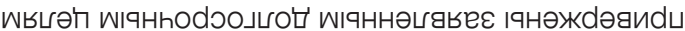

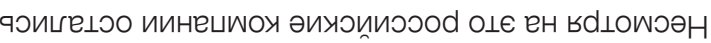

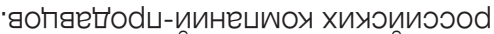

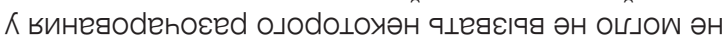

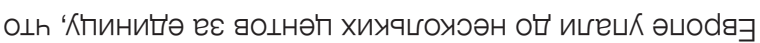

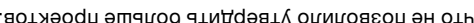

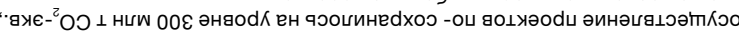

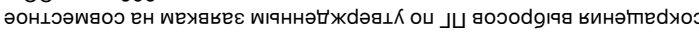

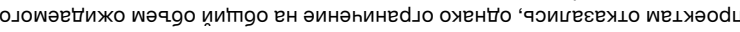

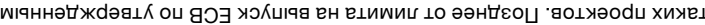

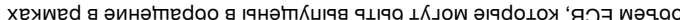
" - ¿

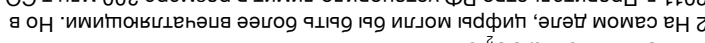

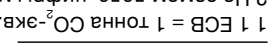

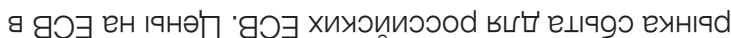

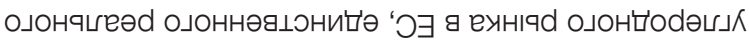

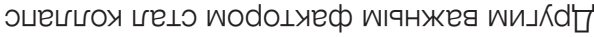

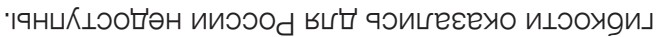

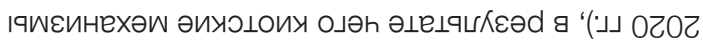

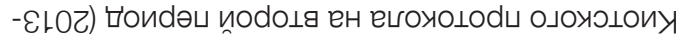

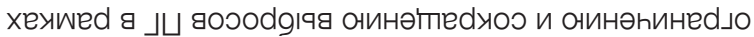

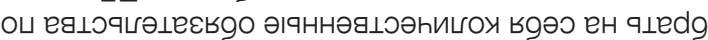
еце

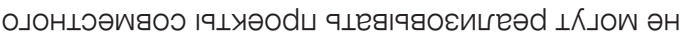

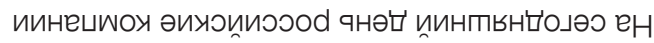

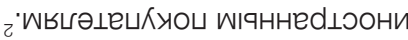

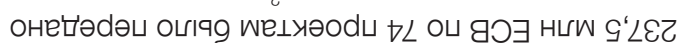

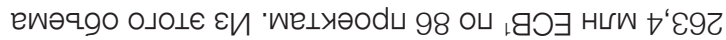

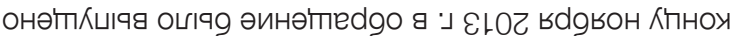

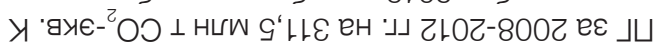

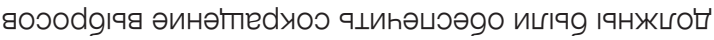
І

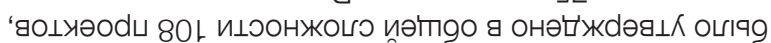

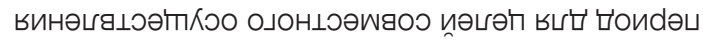

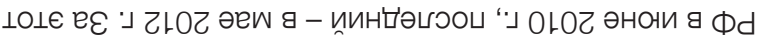

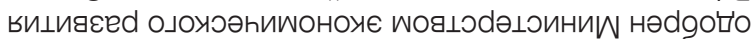

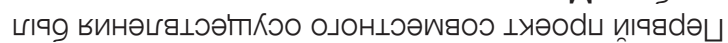

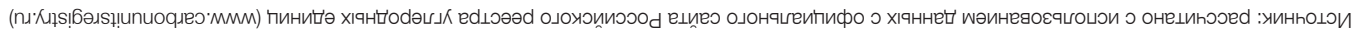

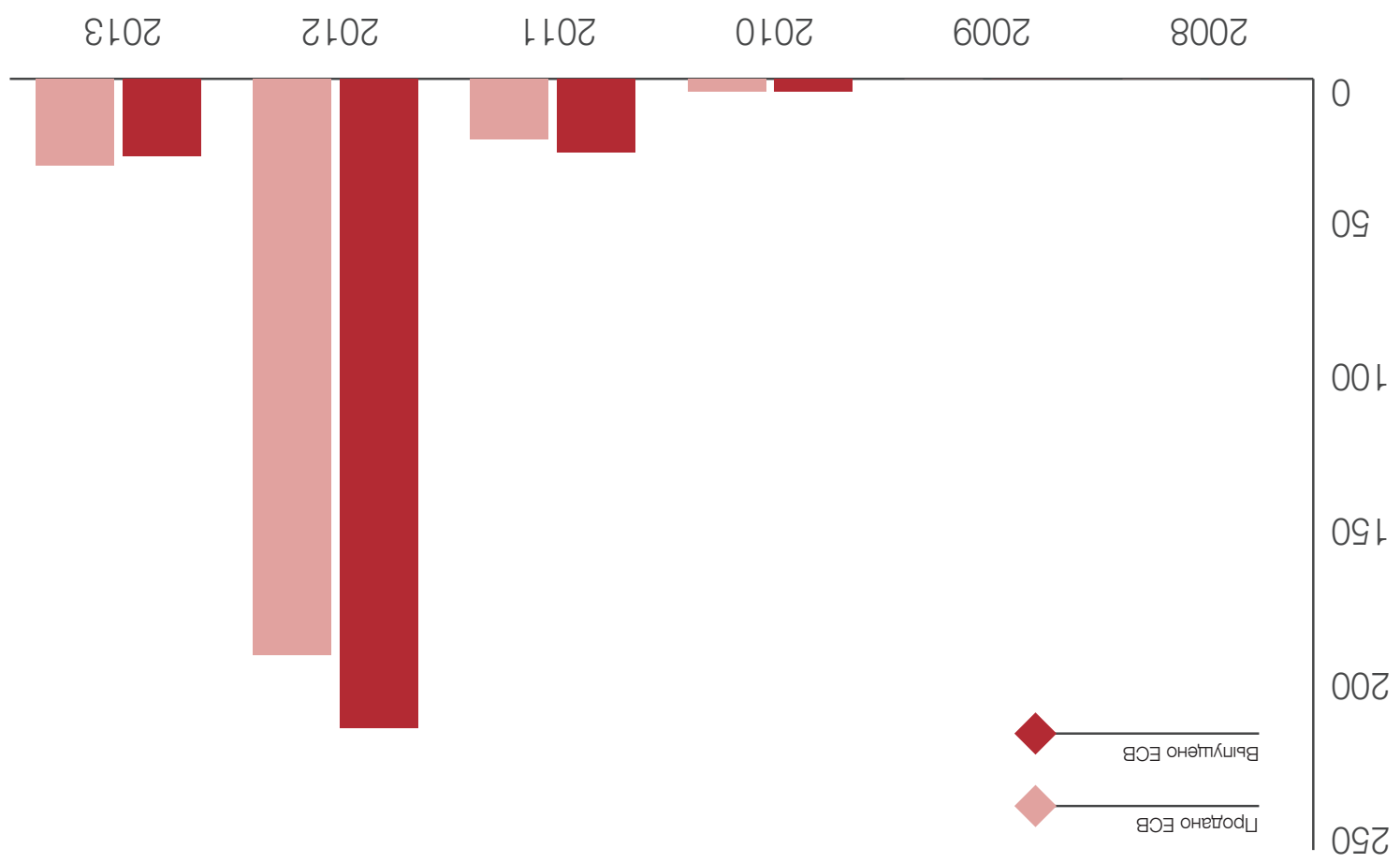

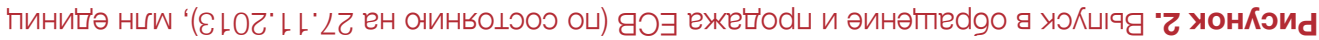




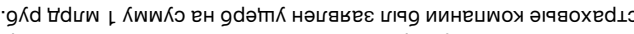

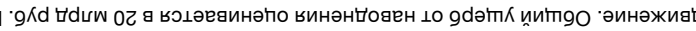

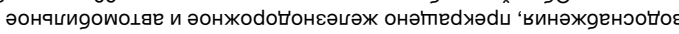

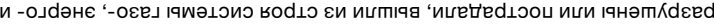

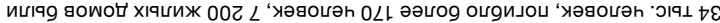

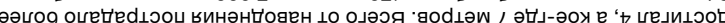

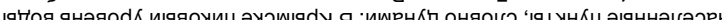

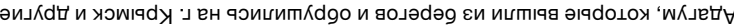

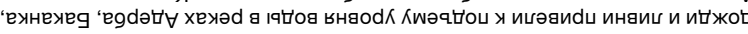

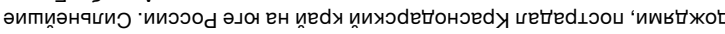

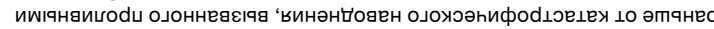

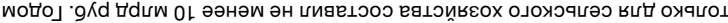

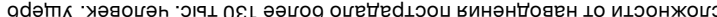
иə)

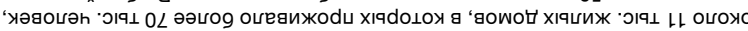

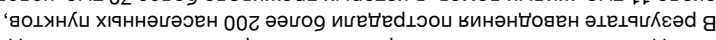

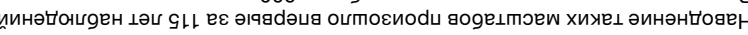

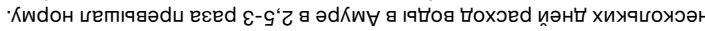

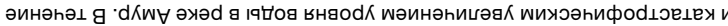
иพех

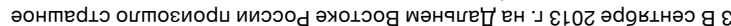

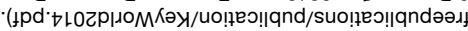

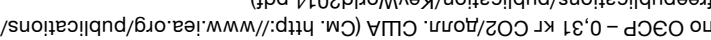

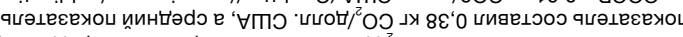

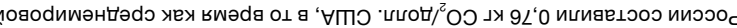

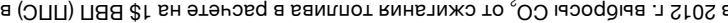

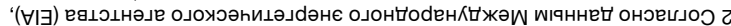

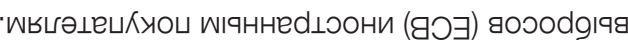
винә'пе

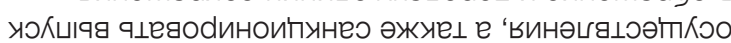

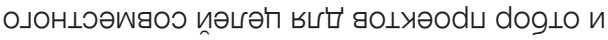

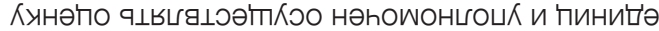

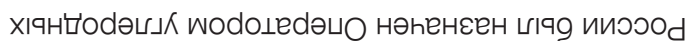

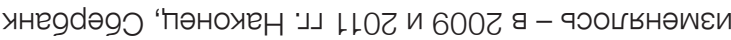

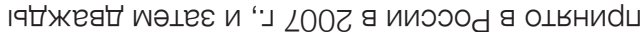

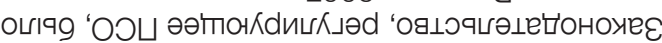

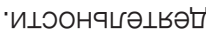

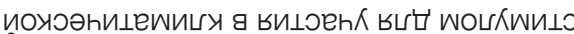

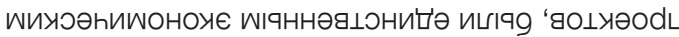

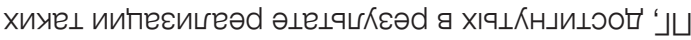

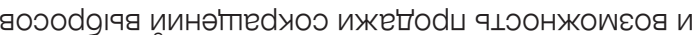

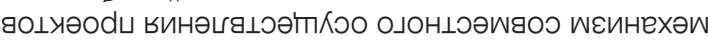

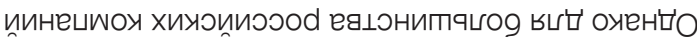

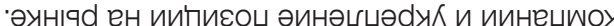

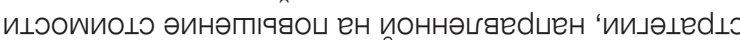

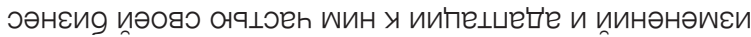

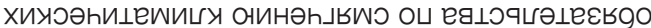

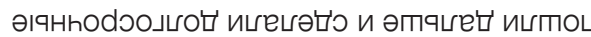

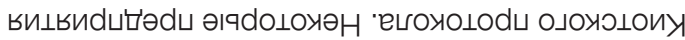

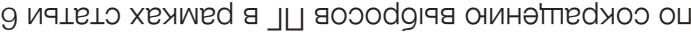

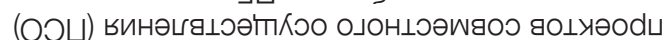

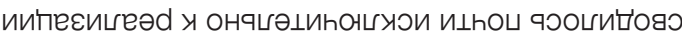

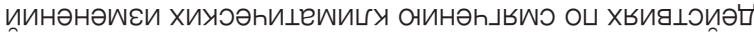

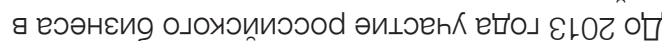

ецешиц् винәнәшєи оинәһ」ьшо

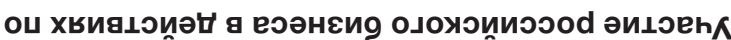

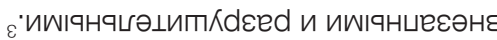

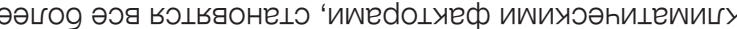

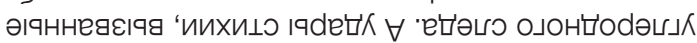

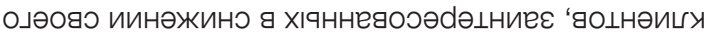

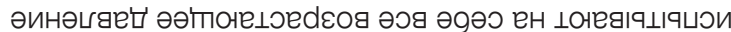

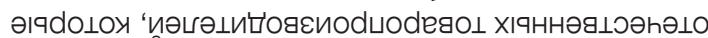

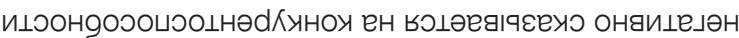

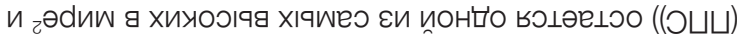

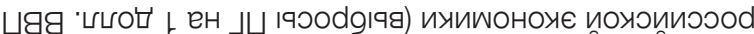

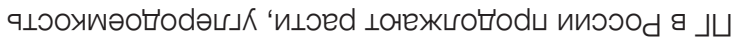

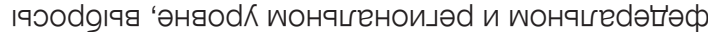

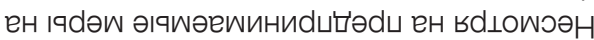

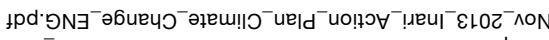

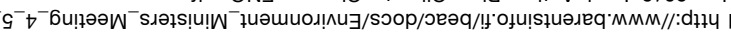

」

иdени а ецәяо о олохоәһиц्रd $\forall$-oda

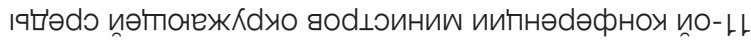

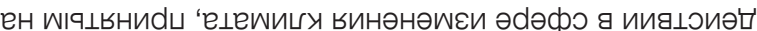

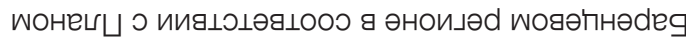

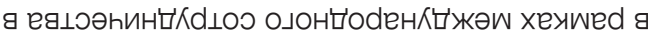

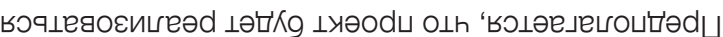

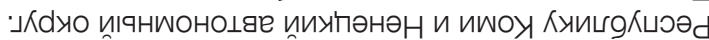

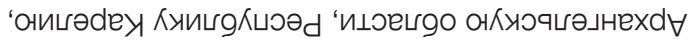

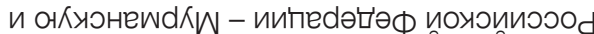

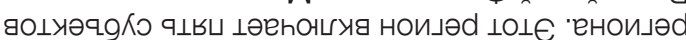

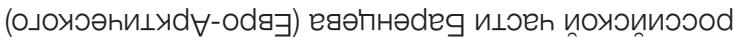
ьப'ப' ии

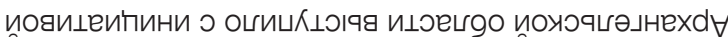

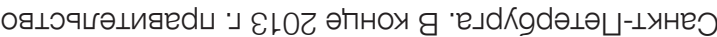

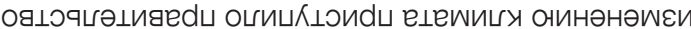

х ииПе

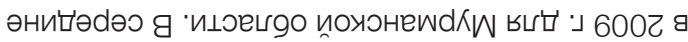

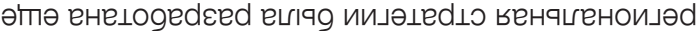

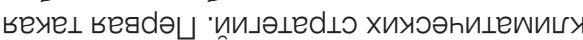

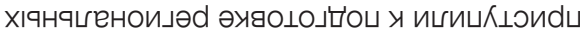

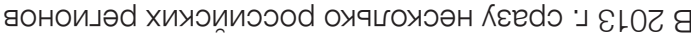
"едцоивєох

о

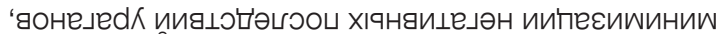

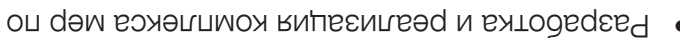

‘ицонаицуе ионнияец

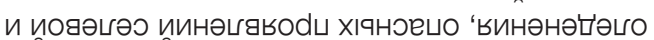

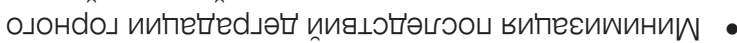

؛енеәуо олояодиN вняодК винәпाяяоч и иинәнц'ояен ' ехой хьияогоК а иинәнцовен ецой ьинәһицәяК

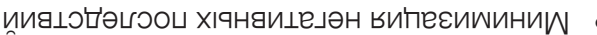

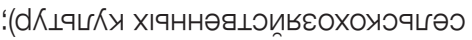

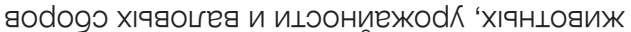

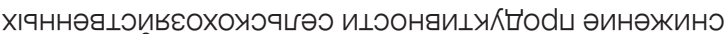

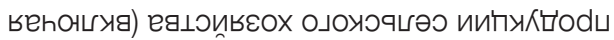

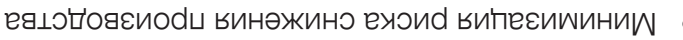

؛ийеdәむәәФ иохоииоวод

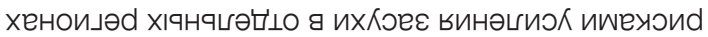

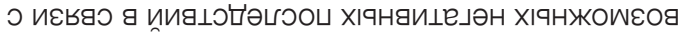

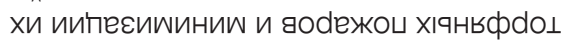

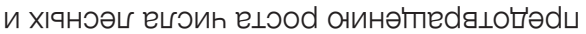

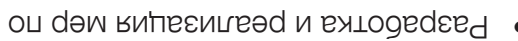

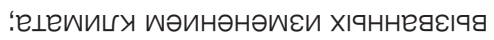

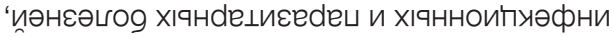

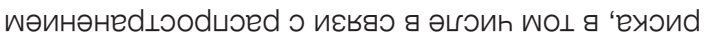

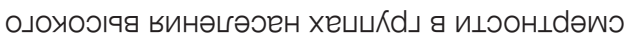

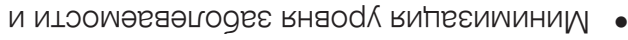

:уеу 'wejoduog

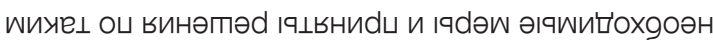

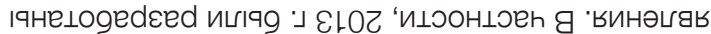

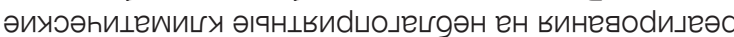

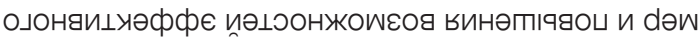

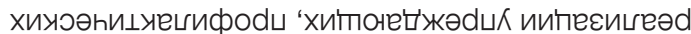

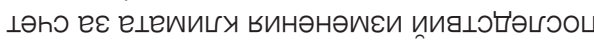




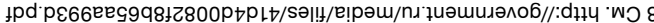
' १เея

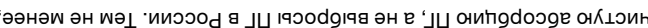

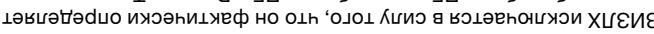

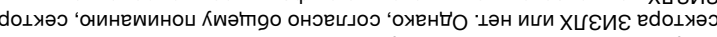

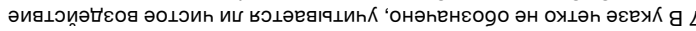

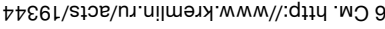

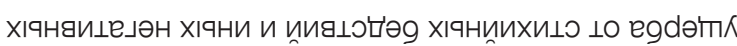

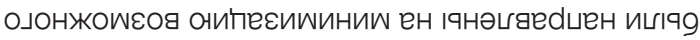

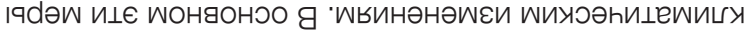

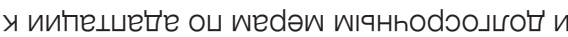

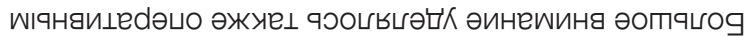

ப в

әинә'пе

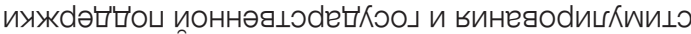

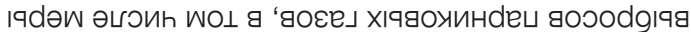

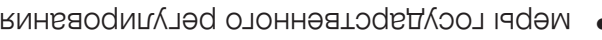

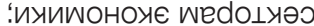

оப ل」 воэodgıя винәтпе

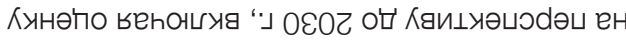

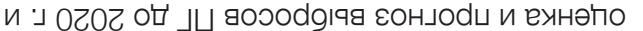

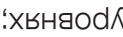

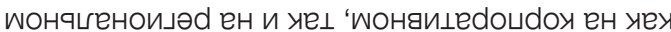

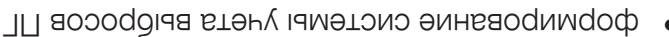

:Иıวеh

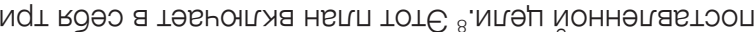

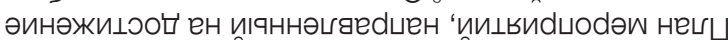

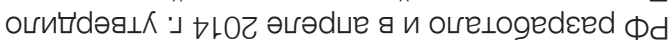

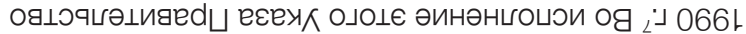

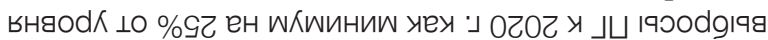

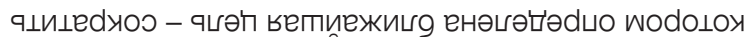

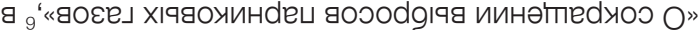

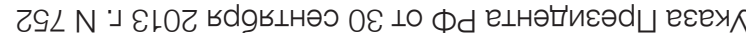

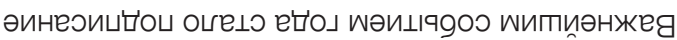

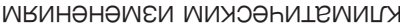

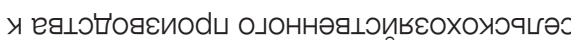

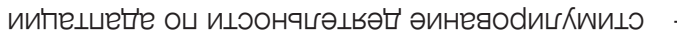

:еgцОивєох

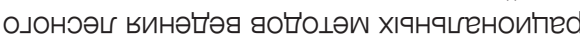

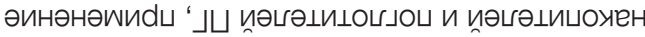
уех яоวәч ея॰วәһеу әинәпाчяоப и енеdхо

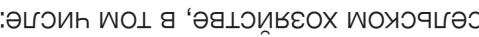

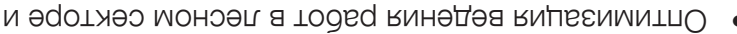

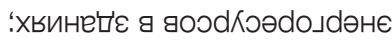

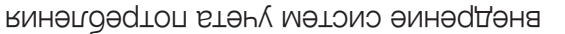

؛иинечwох XIवноияdəวо」dәнє

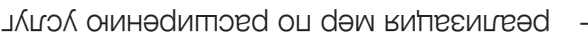

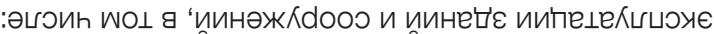

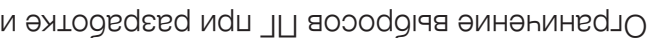

؛

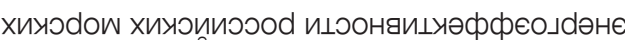
оинәпाчgоч оப dəw exıogedeed -

'иипеияе иочонеЏжеd」 а 」」 воэоdgıя

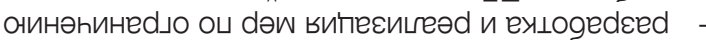

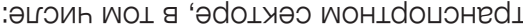

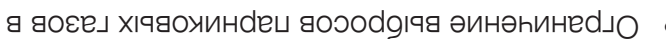

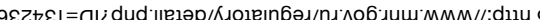

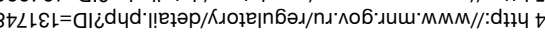

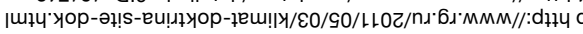

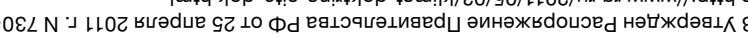

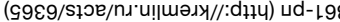

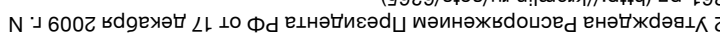

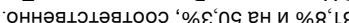

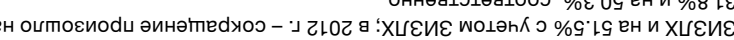

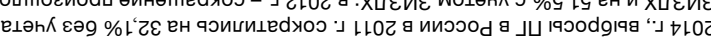

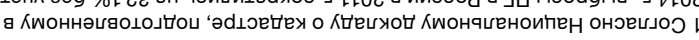

؛Hе_OW

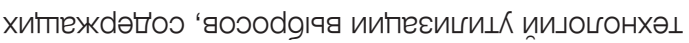

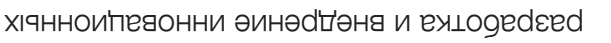

:ии」dәнє ионwоце ьинеgовqчочои әяоноо ен йцогонхәц хІवнноипеяонни әинәdџәня -

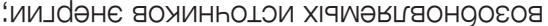

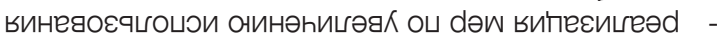

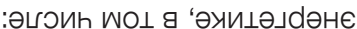

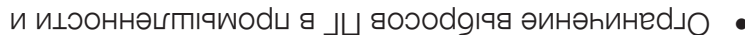

؛」 gоэоdgıя әинәжино и әинәһинед」о

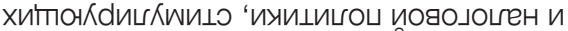

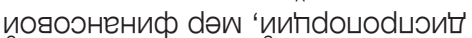

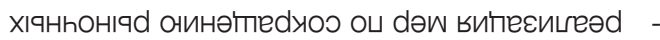

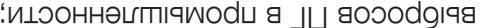

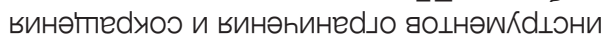

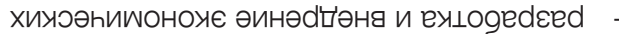

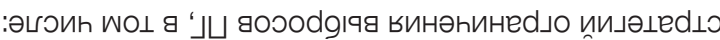

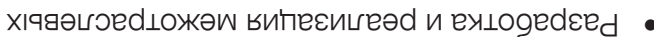

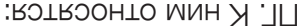
gоวоdgıя әинә'пе

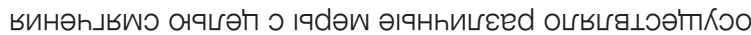

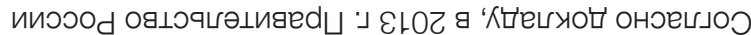

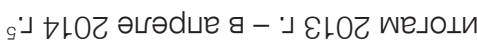

оப 'иоdoıа '

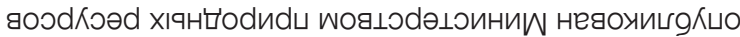

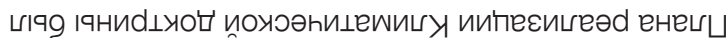

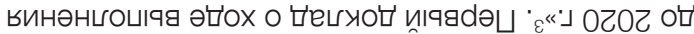

ఛоиdәப ен Іяни

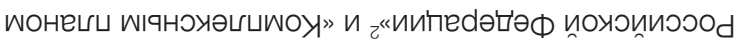

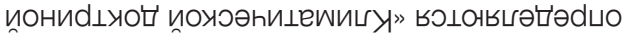

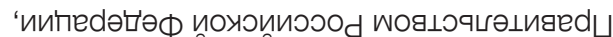

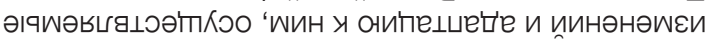

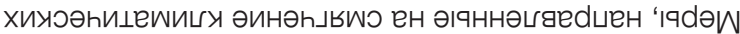

мин х випецчете

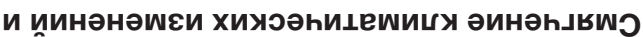

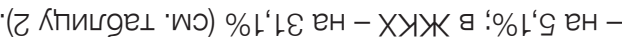

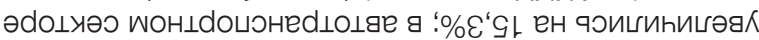

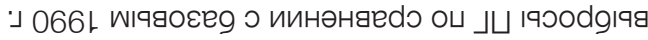

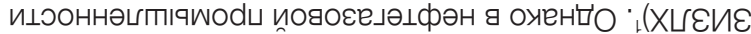

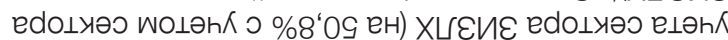

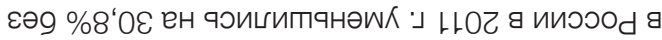

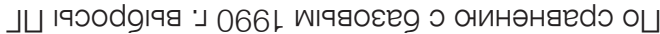

$$
\perp \text { HएW 9'G }
$$

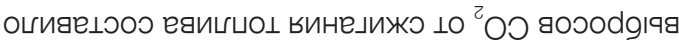

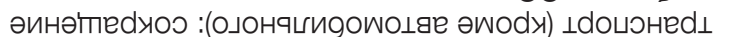




\begin{tabular}{|c|c|c|c|}
\hline ย'乙દよ & 6'08 & L‘ 19 & 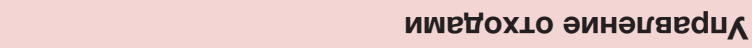 \\
\hline 068 & 乌“เE & 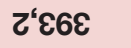 & ичวеdıо әиһоdப \\
\hline レ‘ட\&เ & 9‘てレ & L'Z6 & 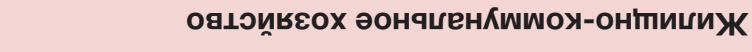 \\
\hline 8'0ع & L'9 & $\varepsilon^{\prime} \nabla G$ & 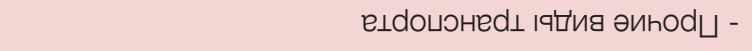 \\
\hline 9‘えL & 乙‘08 & G’OLL & 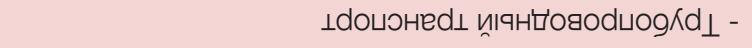 \\
\hline \multirow[t]{2}{*}{ L'90 L } & L’981 & $G^{\prime} \angle L L$ & 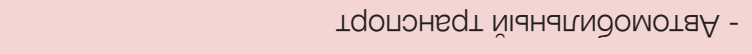 \\
\hline & & & 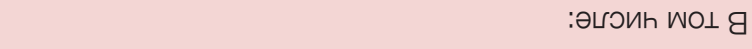 \\
\hline 8'乙8 & 9‘ع8乙 & 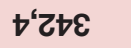 & 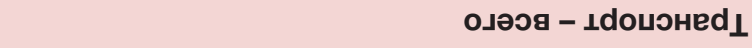 \\
\hline s'Et & เ‘99เ & เ‘6LE & 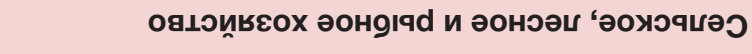 \\
\hline L't9 & ย’てヤト & て’O乙て & 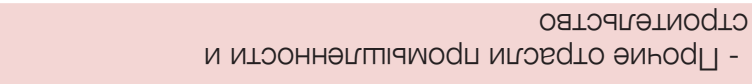 \\
\hline L'69 & 9‘વع & O'L & 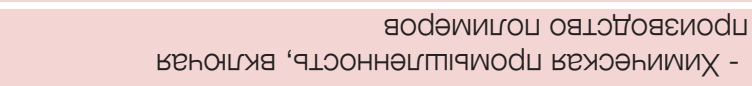 \\
\hline \multirow[t]{2}{*}{ 乙‘OL } & O‘Eヤ L & L'EOZ & 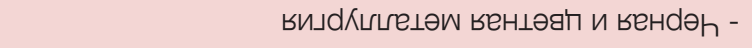 \\
\hline & & & 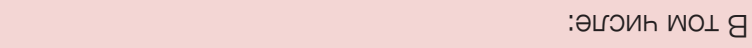 \\
\hline 969 & 6'0Zع & $0 ‘ s<t$ & 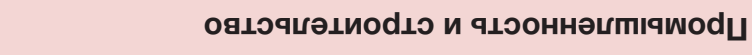 \\
\hline 9‘00 L & $\nabla^{\prime} \varepsilon L$ & $0^{\prime} \varepsilon L$ & 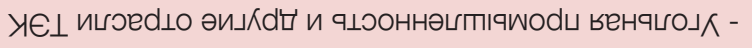 \\
\hline ย’Gト & ヤ゙ヤレカ & $\nabla * 69 \varepsilon$ & 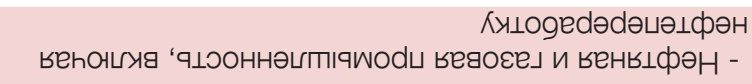 \\
\hline \multirow[t]{2}{*}{ G'OL } & 9‘628 & $0 ‘ 9 \angle L L$ & 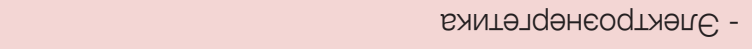 \\
\hline & & & 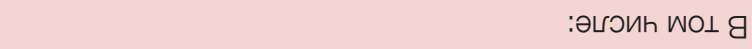 \\
\hline \multirow[t]{2}{*}{$6 ' 18$} & $\nabla^{6}\llcorner L \varepsilon レ$ & G‘809 ا & 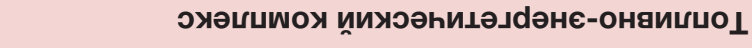 \\
\hline & & & :хин $\varepsilon И$ \\
\hline 乙‘69 & 8‘0Zદ 乙 & 6'เGE $\varepsilon$ & 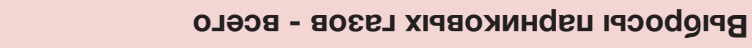 \\
\hline 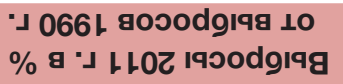 & •レトOZ & ل & 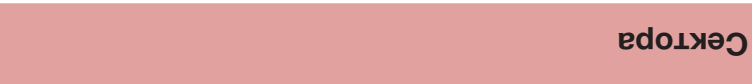 \\
\hline
\end{tabular}

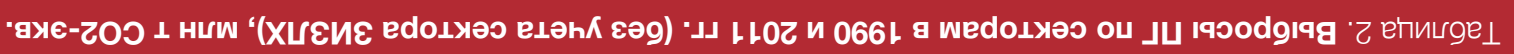

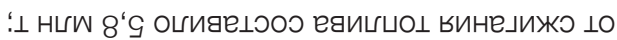

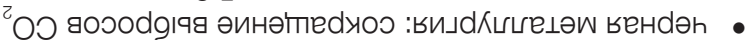

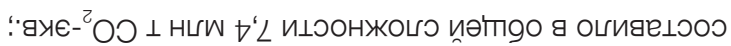

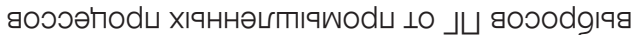
әинәтпе

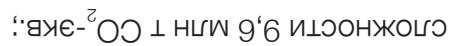

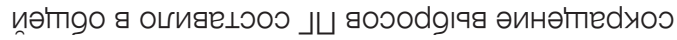
:ояцоивєох әоноәц и әоняІаd ‘әохочьәо•

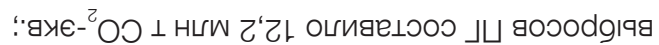
әинәтпе

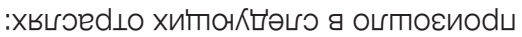

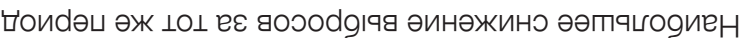

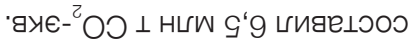

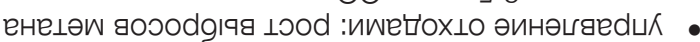

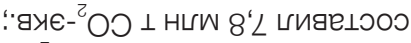

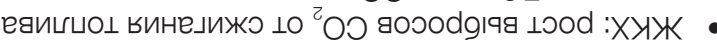


"ave- ${ }^{2} \mathrm{OO} \perp \mathrm{H} \amalg W$ 乙’O

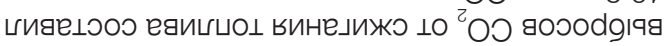

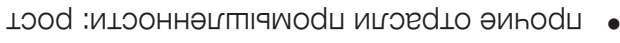

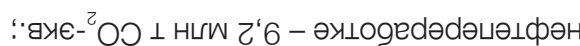

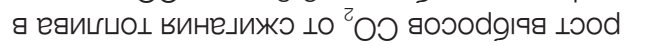

$$
\text { "gye- }{ }^{2} \mathrm{OO} \perp \mathrm{HLW}
$$

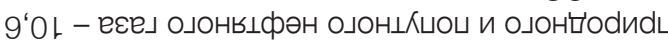

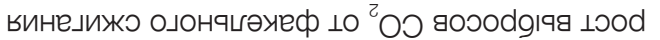

$$
\text { " "aye- }{ }^{2} \mathrm{OO} \perp \mathrm{H} \amalg W \text { L’OL }
$$

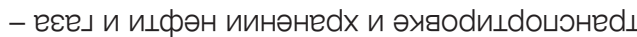

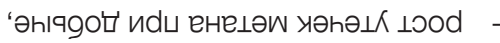

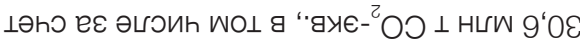

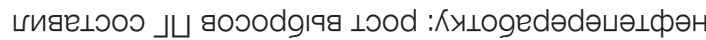

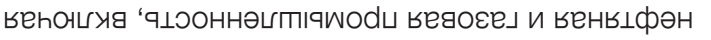

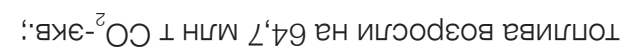

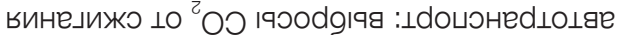

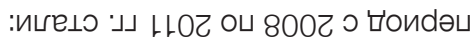

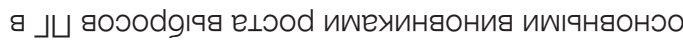

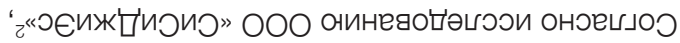

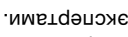

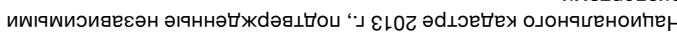

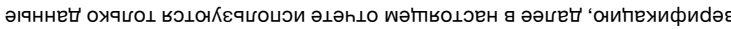

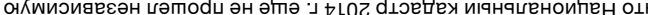

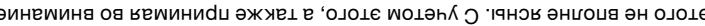

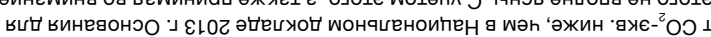

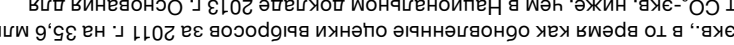

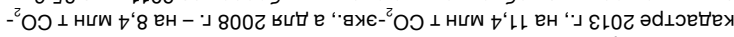

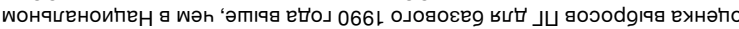
веяон 'ицоонцәен g 'винәжино Кнодоцо а '

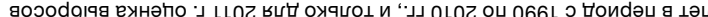

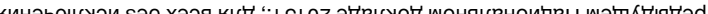
sинен '

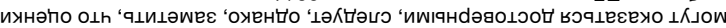

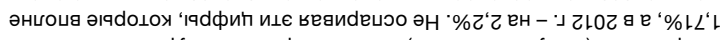

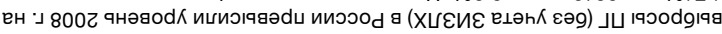

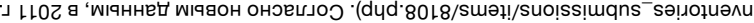

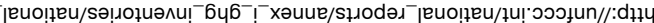
HO० HOO

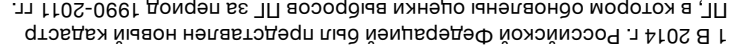

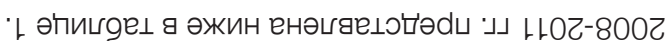

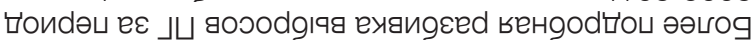

i\%عL'E

ен ици "

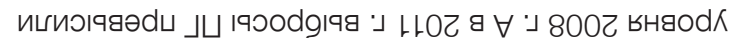

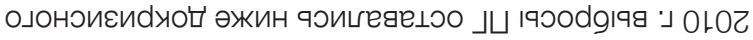

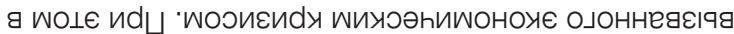

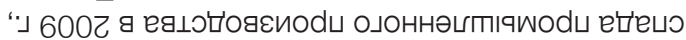

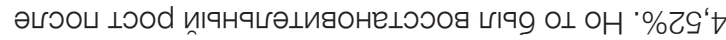

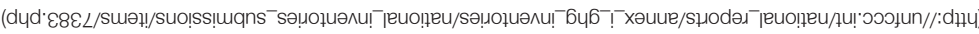

\begin{tabular}{|c|c|c|c|c|}
\hline 8‘0Zદ 乙 & ๕'しเてて & オ‘レてレ & †‘Lع乙 乙 & 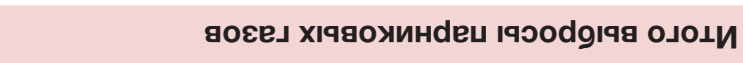 \\
\hline S'0 & L’0 & $8 \times 0$ & $8^{\prime} 0$ & 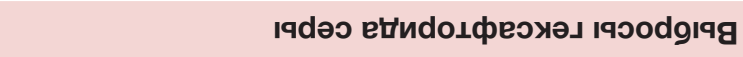 \\
\hline L'6 & 6‘0 & เ’0ト & ヤ゙ヤト & 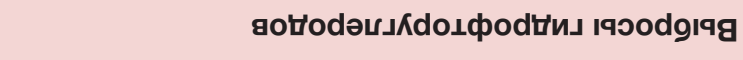 \\
\hline 乌'乙 & L'Z & s'Z & L’E & 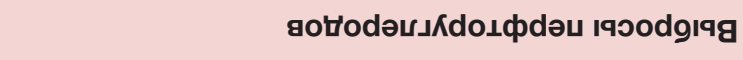 \\
\hline 9‘டL & 8‘とレ & 8‘9レ & て‘9レ & ецове иวихеє І9วоdgІ૧g \\
\hline 9992 & $9^{\prime} \varepsilon L$ & レ゙カL & E'OL & 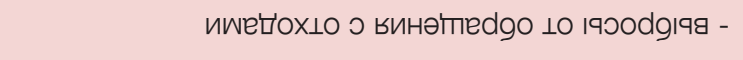 \\
\hline \multirow[t]{2}{*}{ เ’乙৪E } & 乙'69ع & $\nearrow^{\prime}\llcorner\nabla \varepsilon$ & $\angle ’ Z L \varepsilon$ & 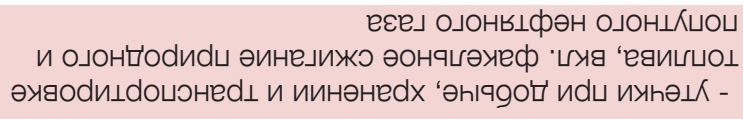 \\
\hline & & & & :хин єи \\
\hline 9'909 & L‘L6t & L't9t & 6 ' $26 t$ & 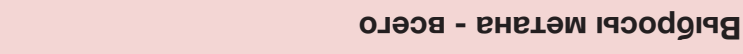 \\
\hline$\varepsilon^{\prime} \angle G L$ & ย’\&૬ เ & L'6EL & $Z^{\prime} \angle G L$ & 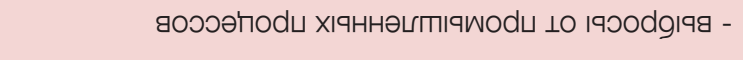 \\
\hline \multirow[t]{2}{*}{ เ’เ6† レ } & く’レレレ & 9 '09ع ᄂ & 8 '92t L & 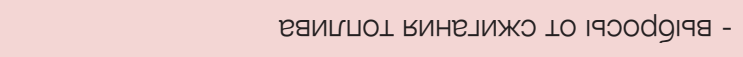 \\
\hline & & & & :хин єи \\
\hline ナ゙†89 レ & 乙‘869 L & t‘9Z9 ᄂ & ع'609 ᄂ & 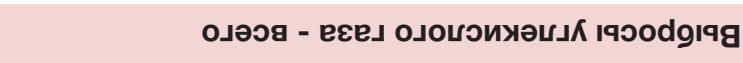 \\
\hline •」トーОZ & נ & - $600 \mathrm{Z}$ & $800 Z$ & gове」 ХІवяохинdе山 І9эоdgІ9g \\
\hline
\end{tabular}

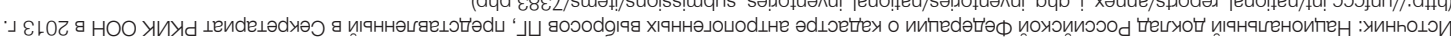




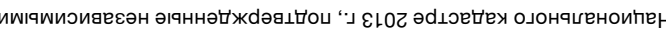

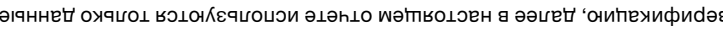

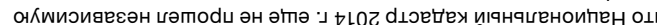

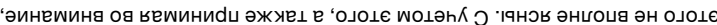

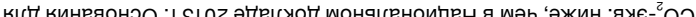
(2)

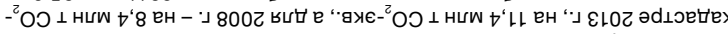

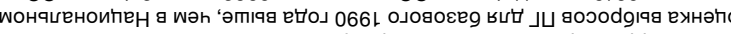
веяон 'ицоон

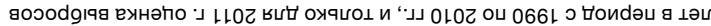

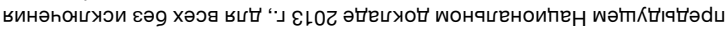
ब wәh 'ә⿴囗十

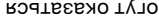

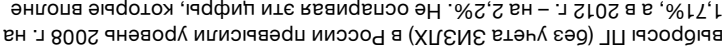

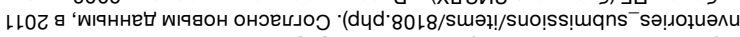

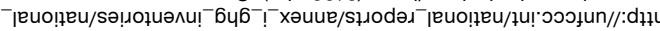

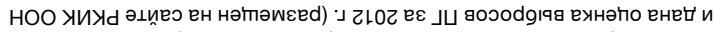

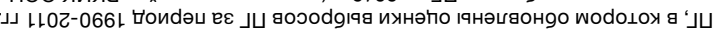

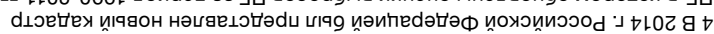

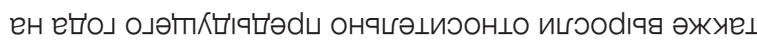

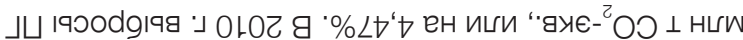

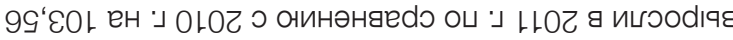

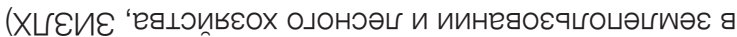

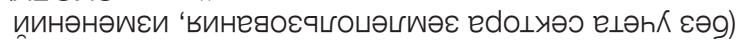

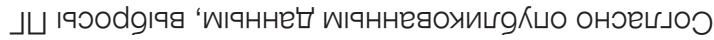

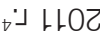

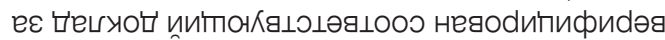

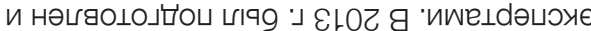

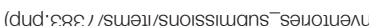

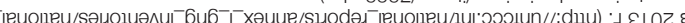
HOO > > И४

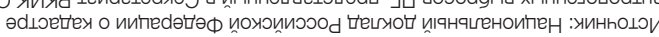

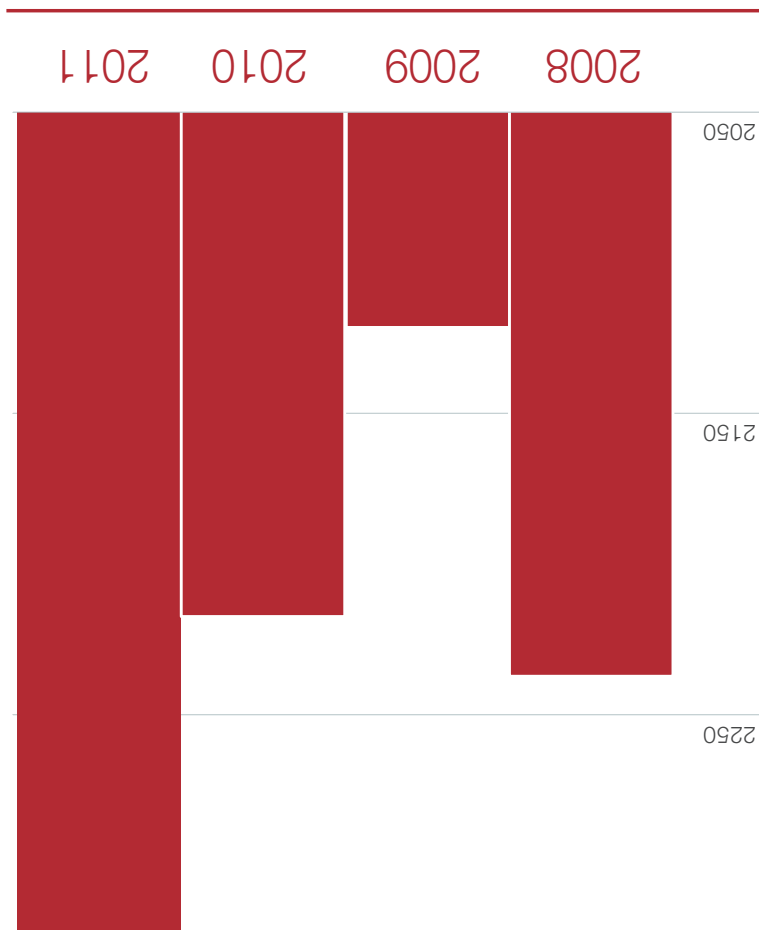

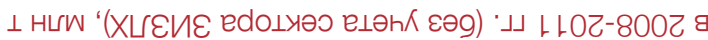

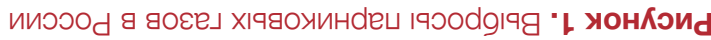

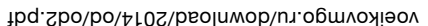
//:dџџ - · †

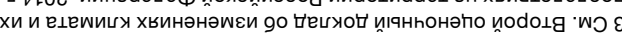

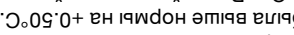

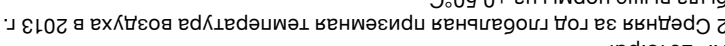

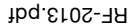

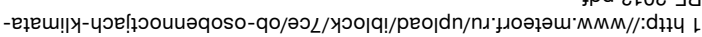

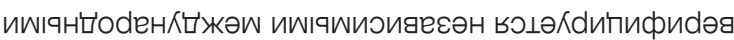

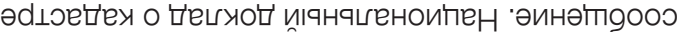

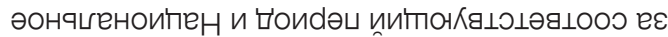

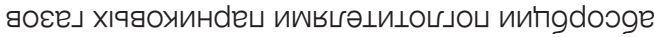

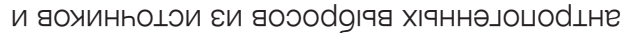

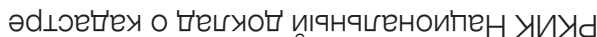

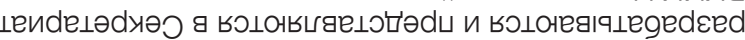

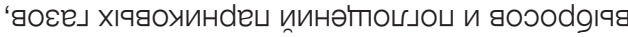

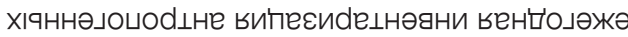

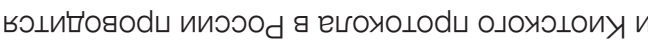

(УИУ્) ецешицг иинәнәшєи 90 НОО ийнәанох

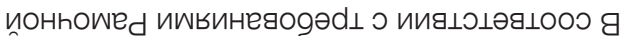

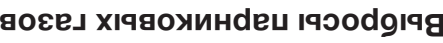

$\%$ L'G eH ИLИ

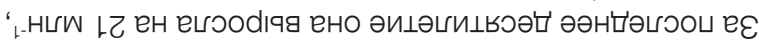

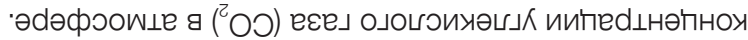

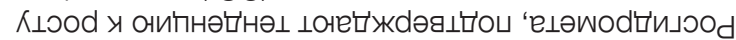

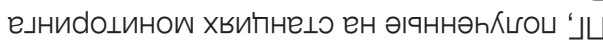

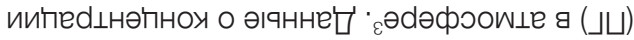
gове」 ХІवgохинdеч иипеdцнәПноу әинәнәшєи

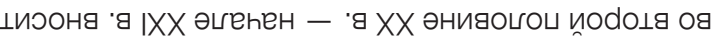

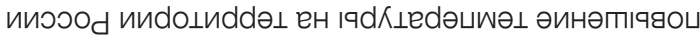

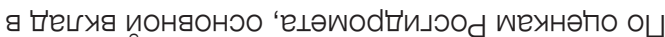

'I9wdoH

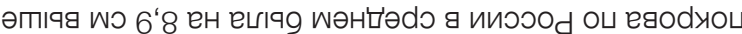

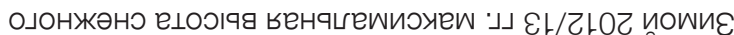

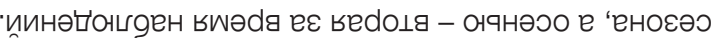

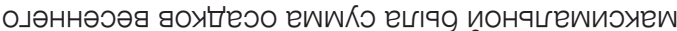

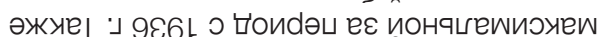

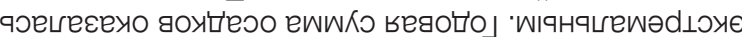

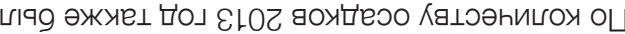

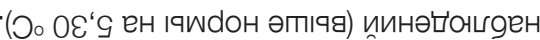

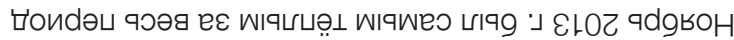

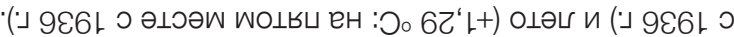

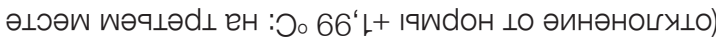

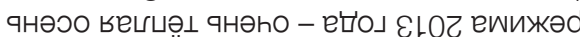

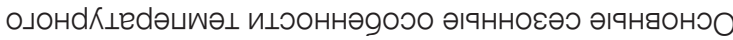

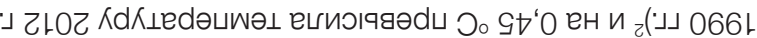

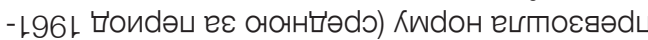

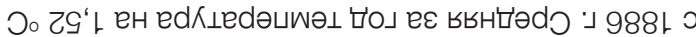

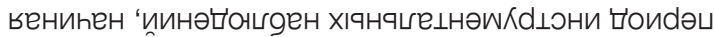

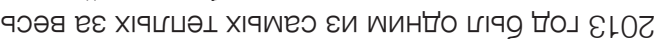

'ı

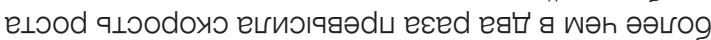

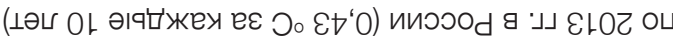

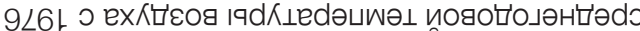

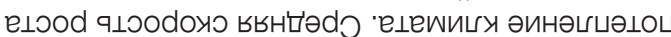

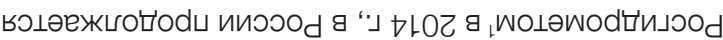

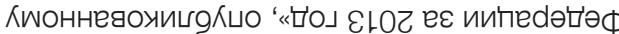

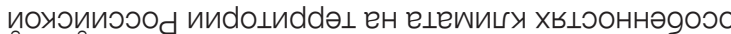

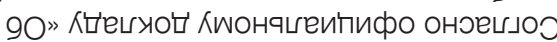

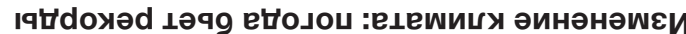


วИด

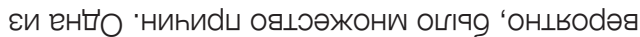

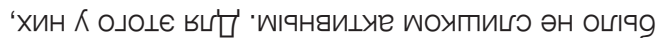

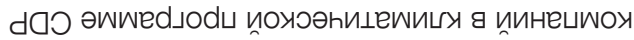

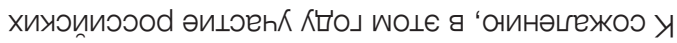

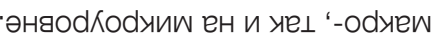

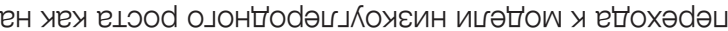

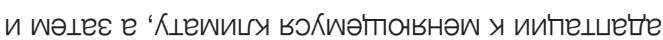

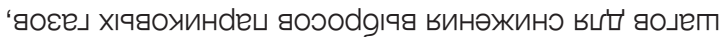

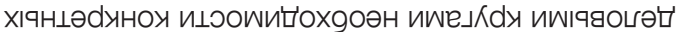

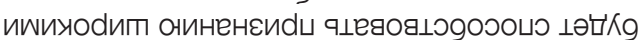

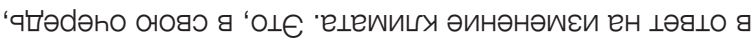

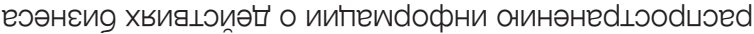

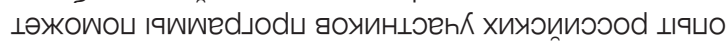

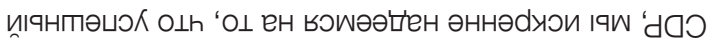

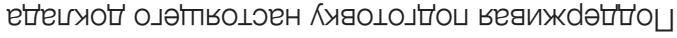

Иинәнәшеи

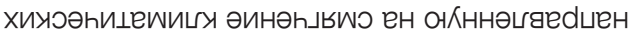

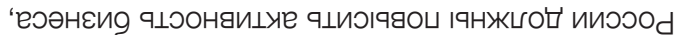

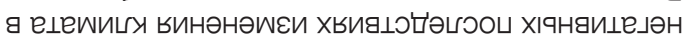

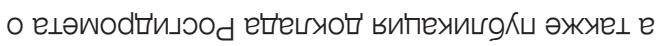

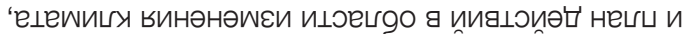

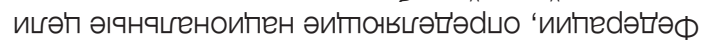

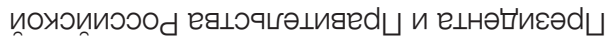

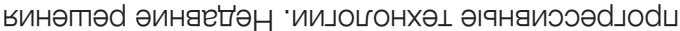

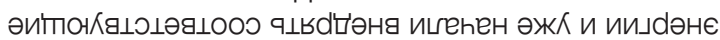

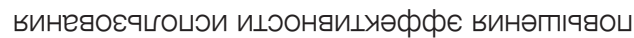

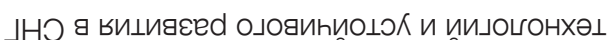
XIव виэวо

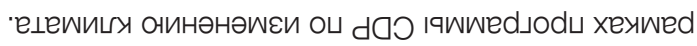

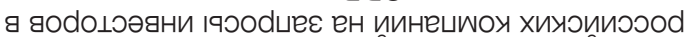

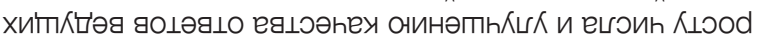

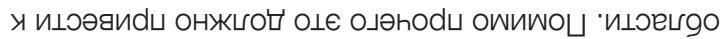

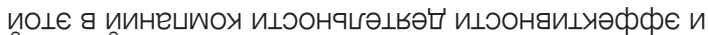

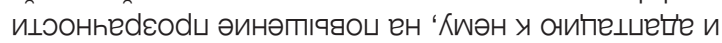

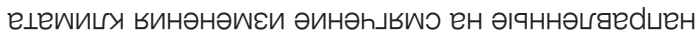

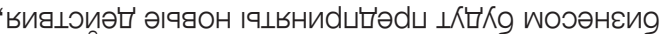

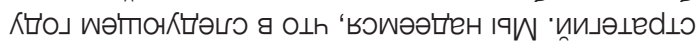

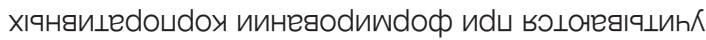

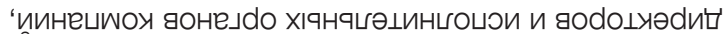

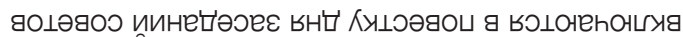

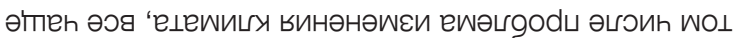

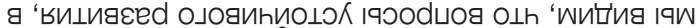

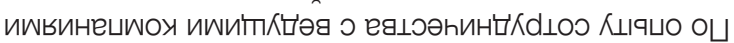

•вициявеd

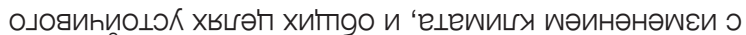

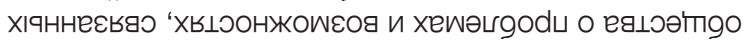

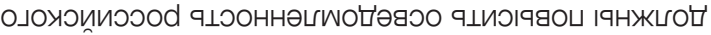

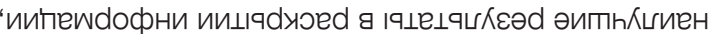

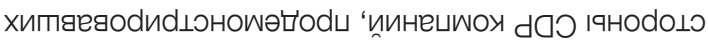
оว әиненєиdப и О」

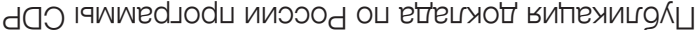

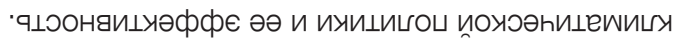
иəовว ৭

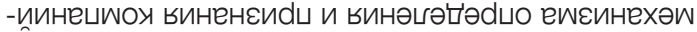

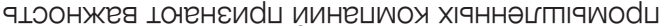

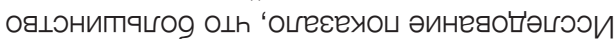

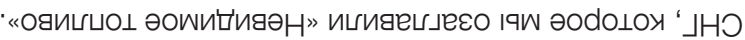

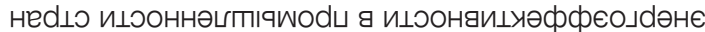

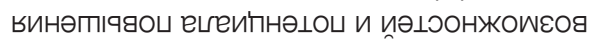

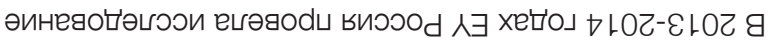

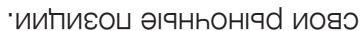

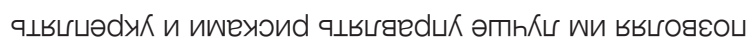

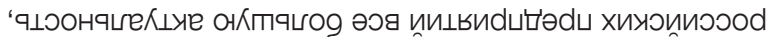

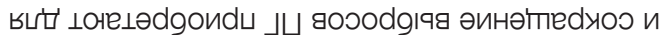

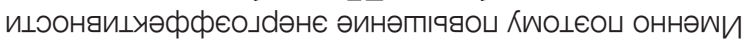

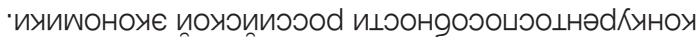

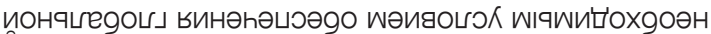

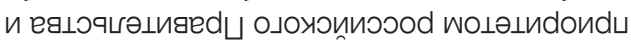

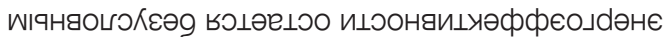

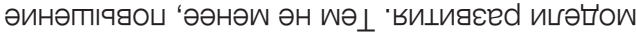

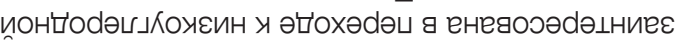

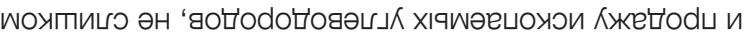

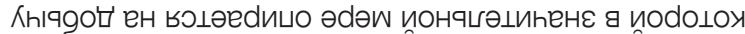

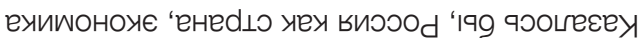

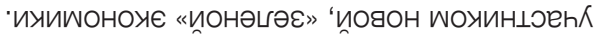

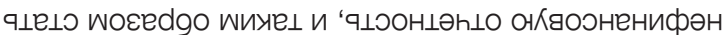

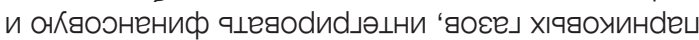

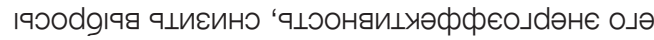

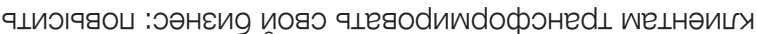

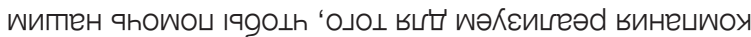
вень'оdeнК'

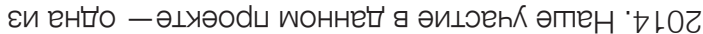

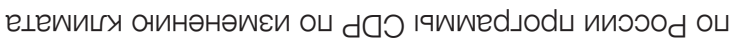

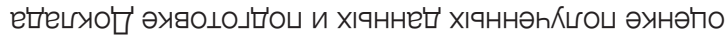

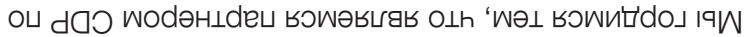




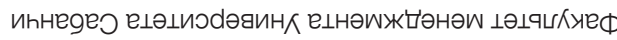

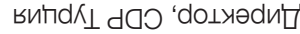

'ұелел еs|әw

"ец्रәоdч ойевинелdo

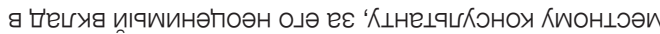

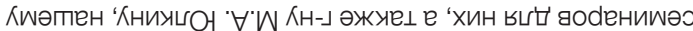

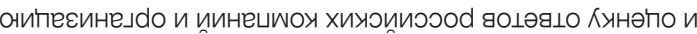

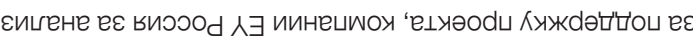

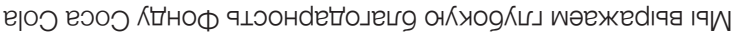

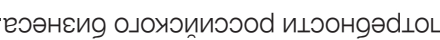

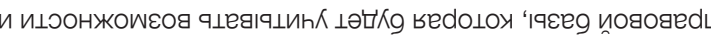

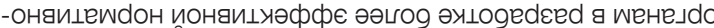

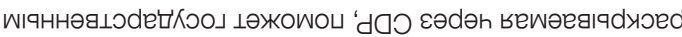

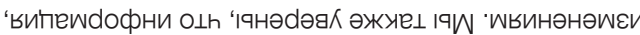

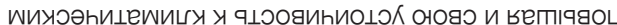

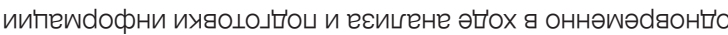

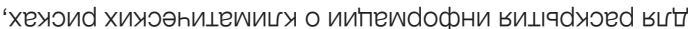

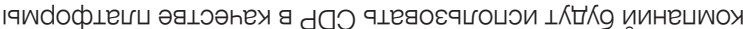

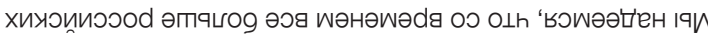

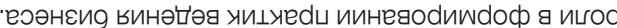

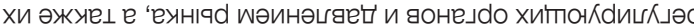

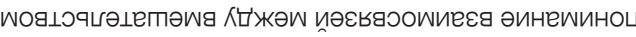

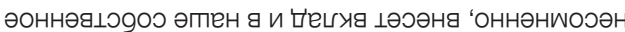

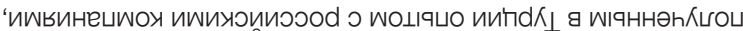

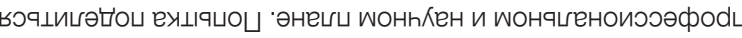

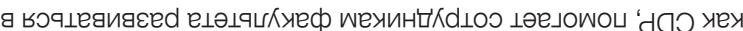
'

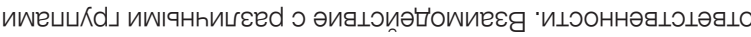

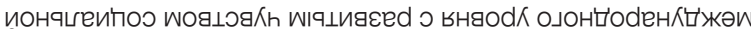

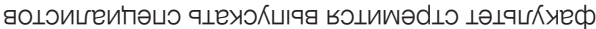

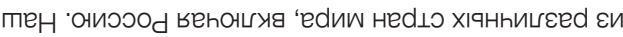

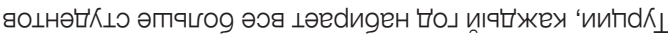

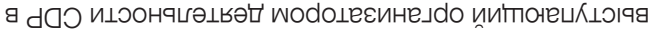

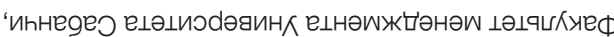

'Иинәнәшеи

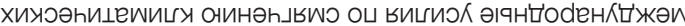

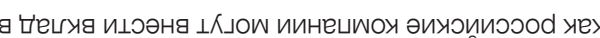

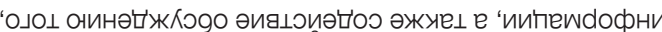

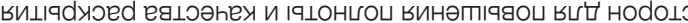

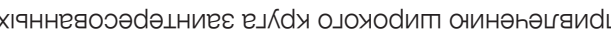
оч 'ий

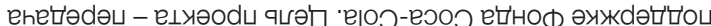

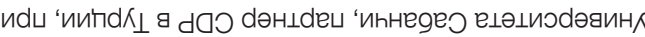

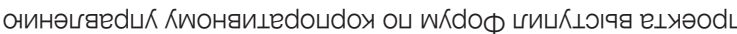

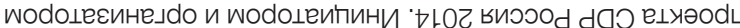

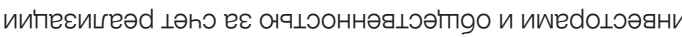

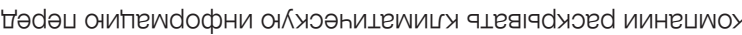

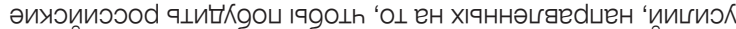

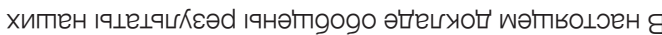

'ецеพицт्र ОИнәнәшєи

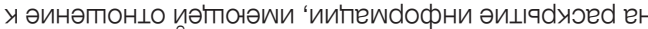

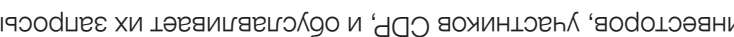

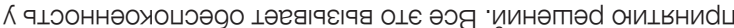

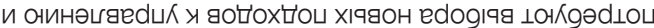

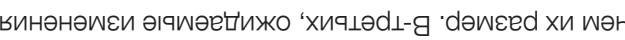

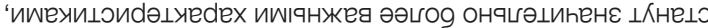

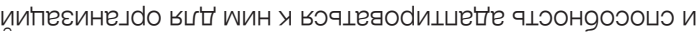

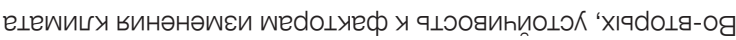

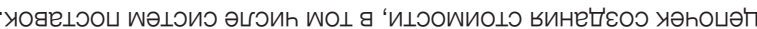
иипеєи $/$ К

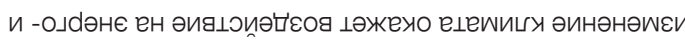

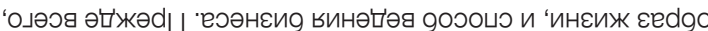

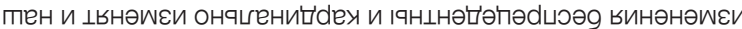

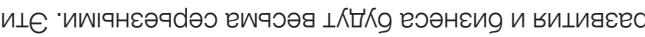

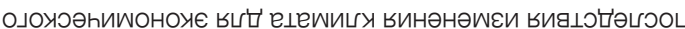

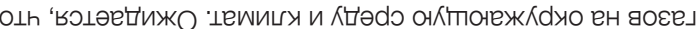

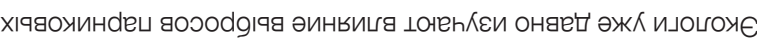

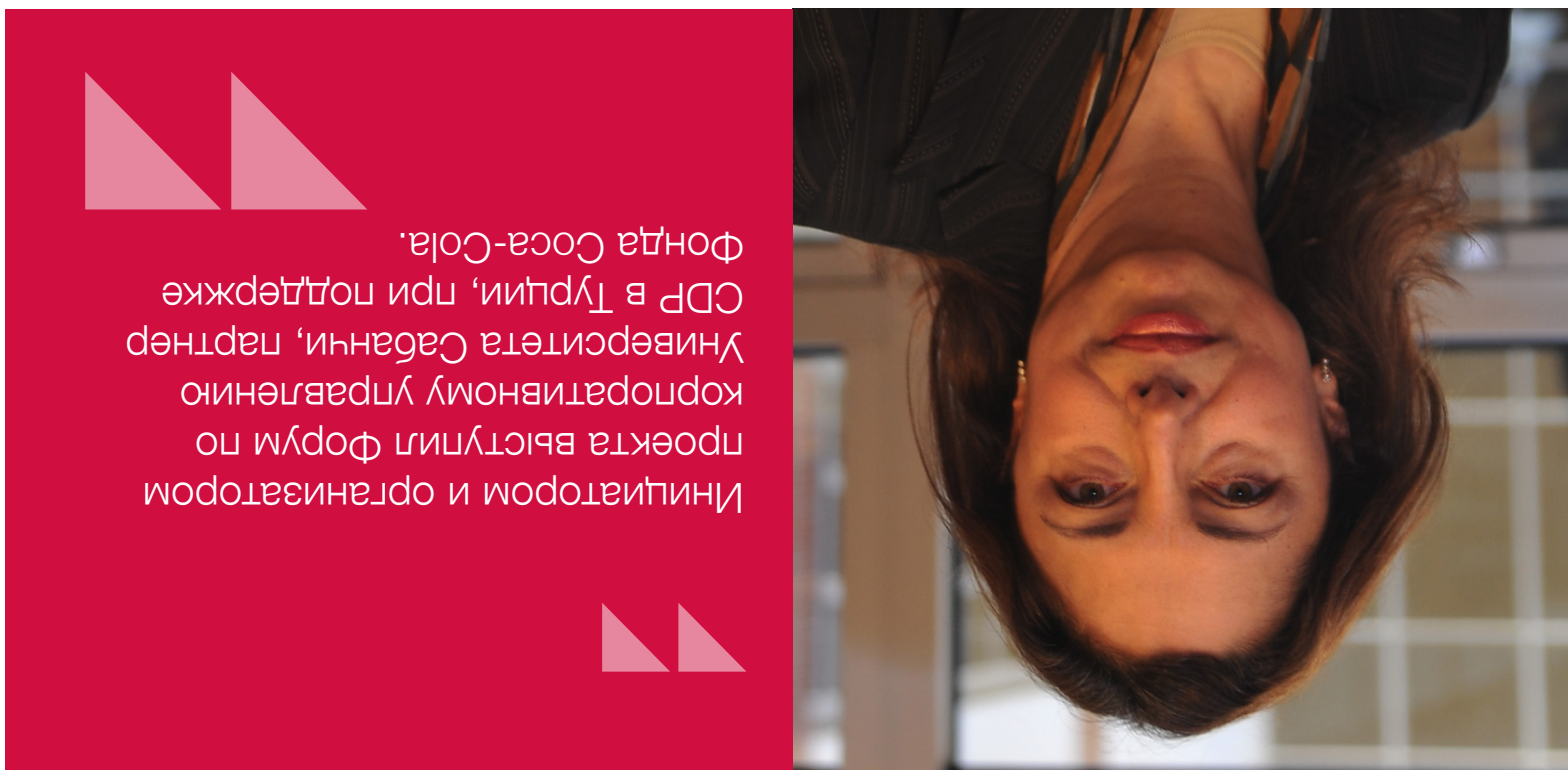




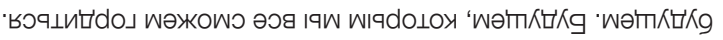

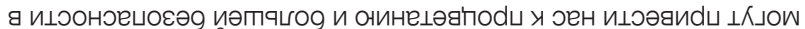

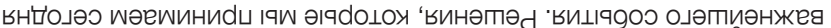

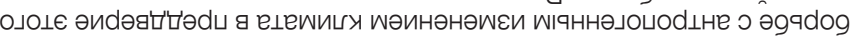

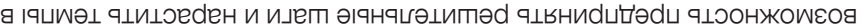

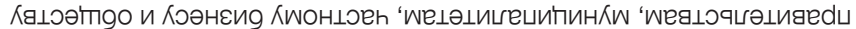

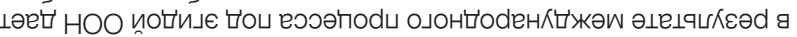

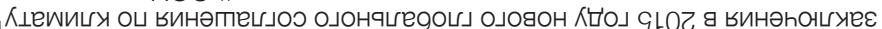

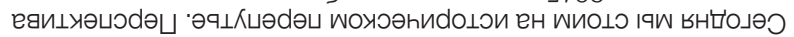

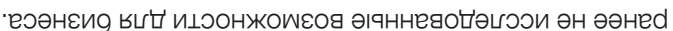

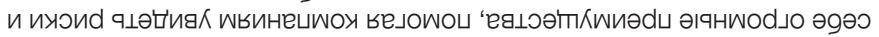

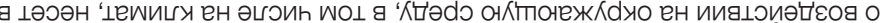

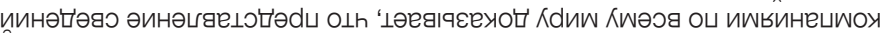

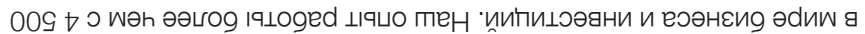

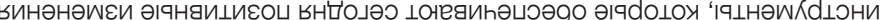

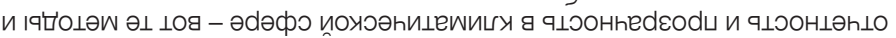

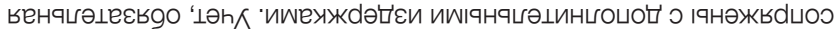

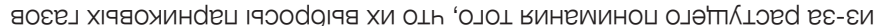

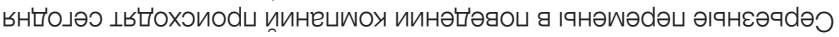

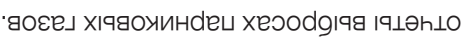

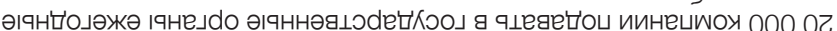

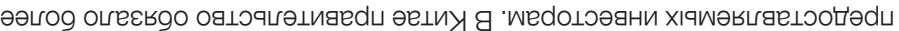

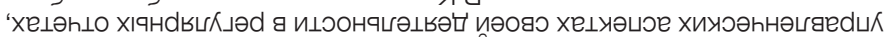

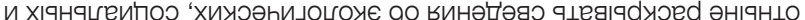

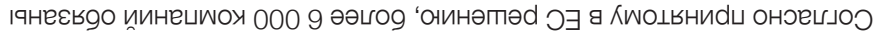

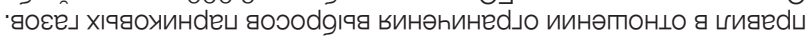

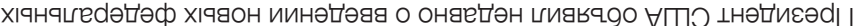

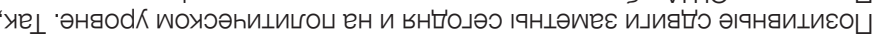

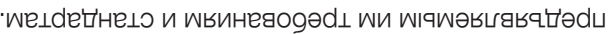
иเеяоя

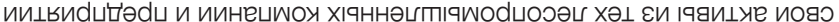

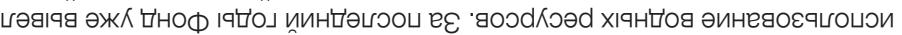

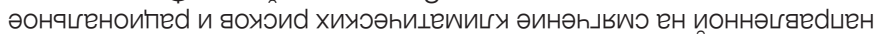

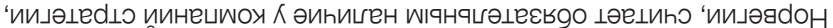

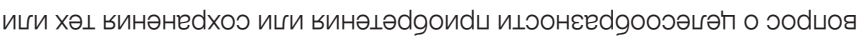

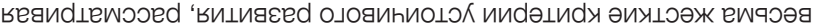

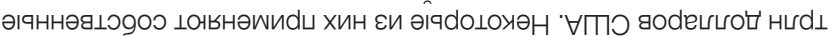

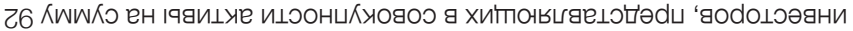

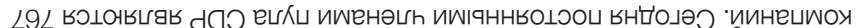

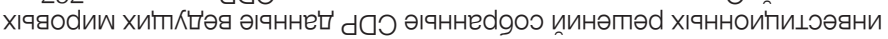

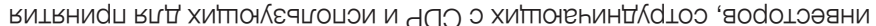

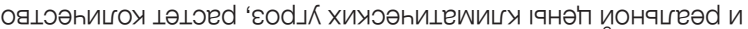

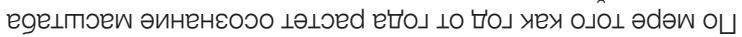

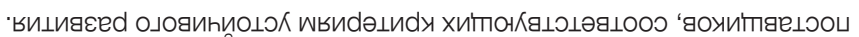

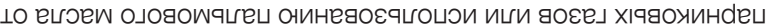
goวodgıя инә乌пе

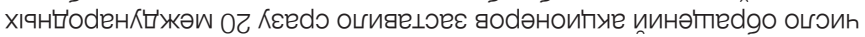

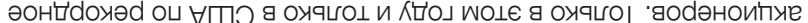

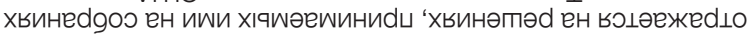

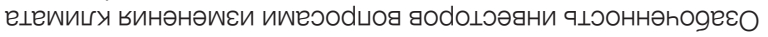

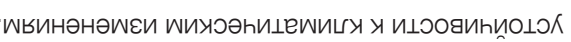

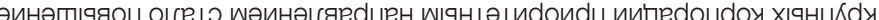

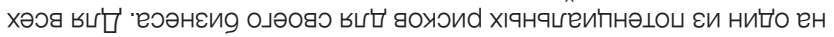

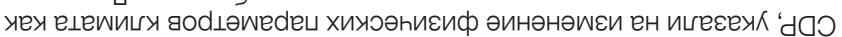

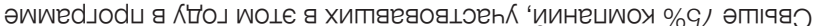

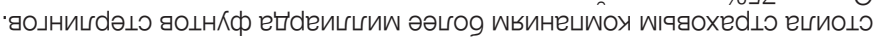

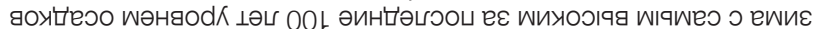

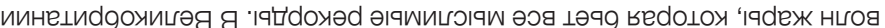

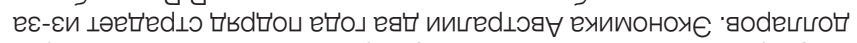

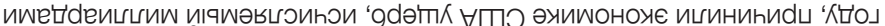

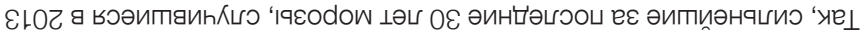

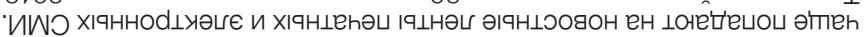

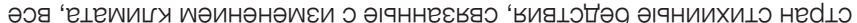

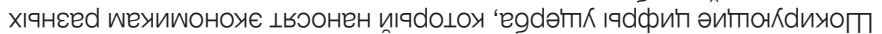

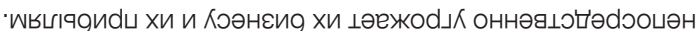

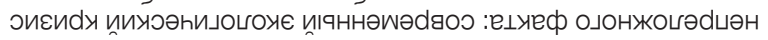

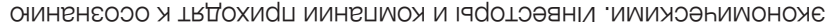

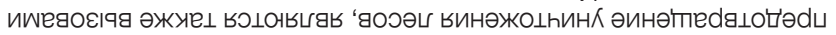

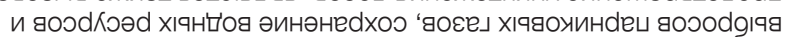
әинәтпе

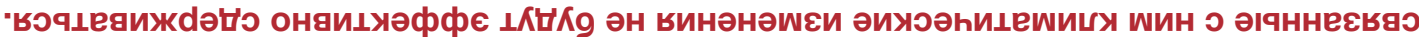

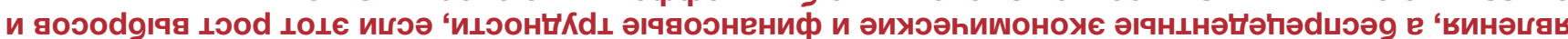

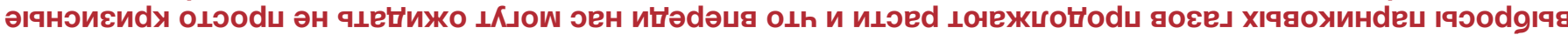

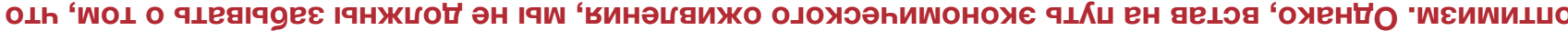

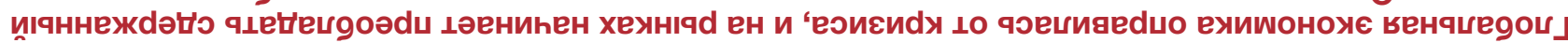
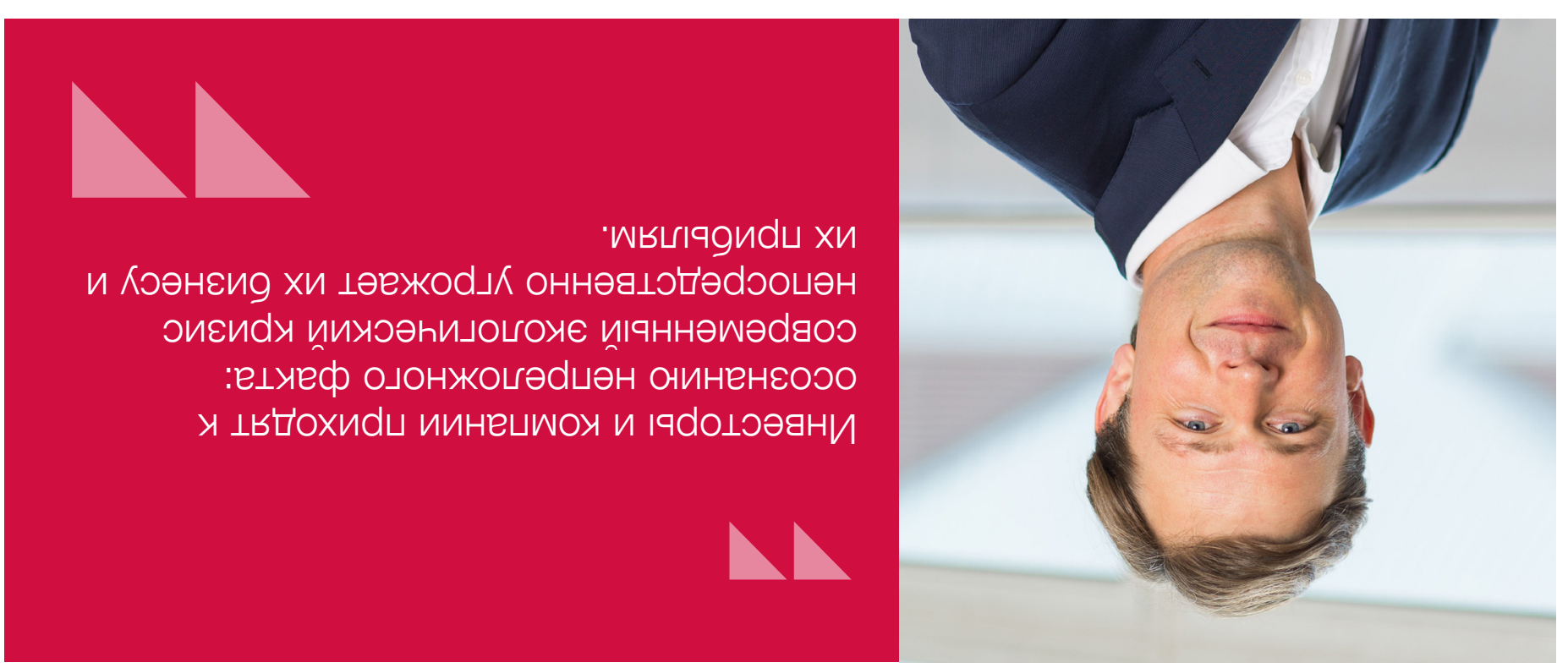

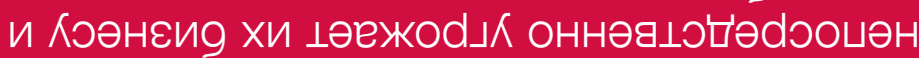

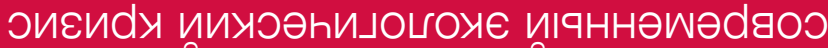

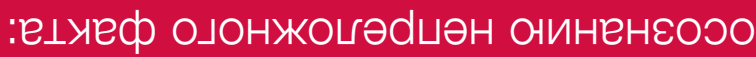

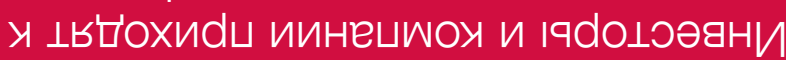

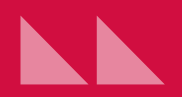




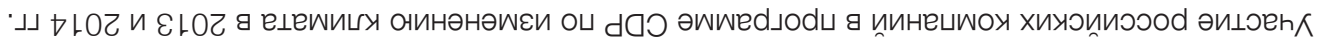

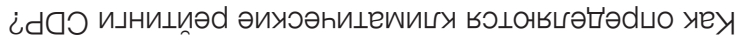

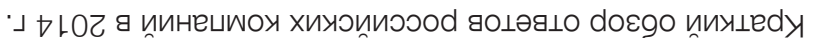

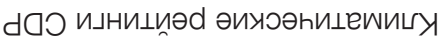

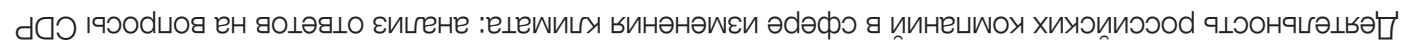
ииоวод в ииПнәШнәц әихоәһицеพицत्र әІаняоноО

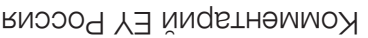
әинәธ๋әя

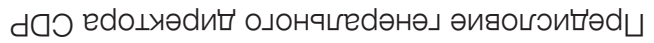


$\cdots \perp$ -

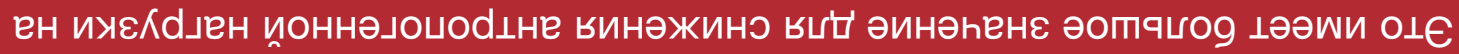

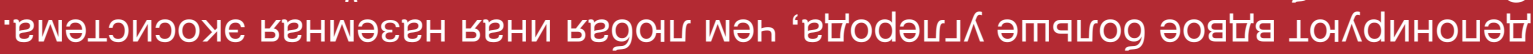

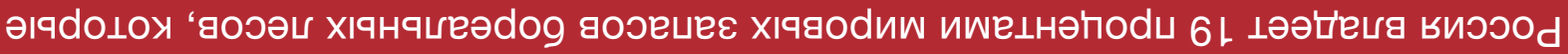

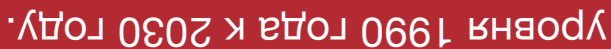

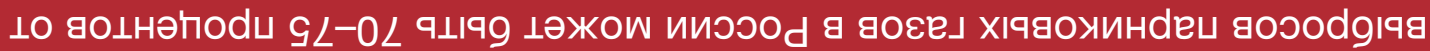

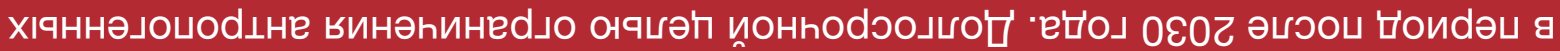

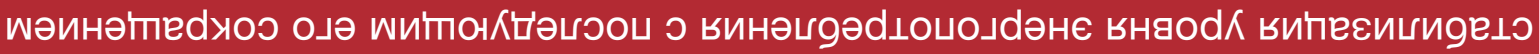

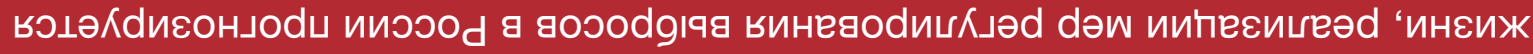

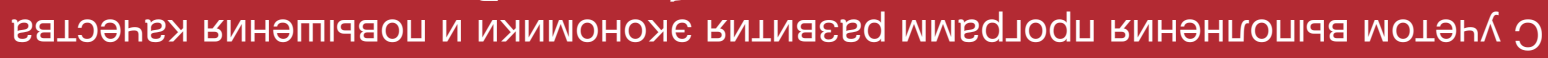

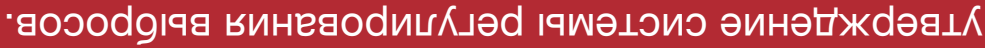

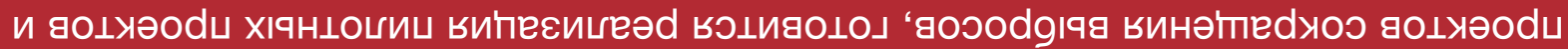

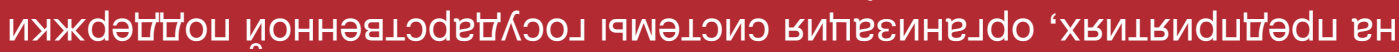

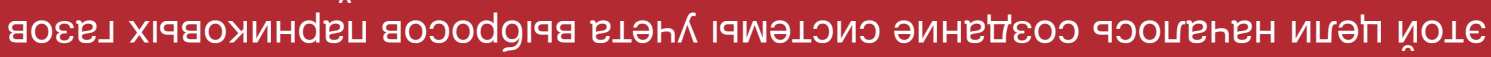

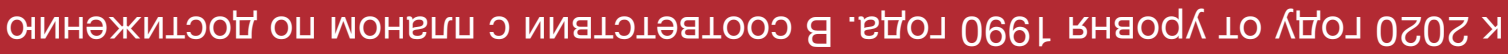

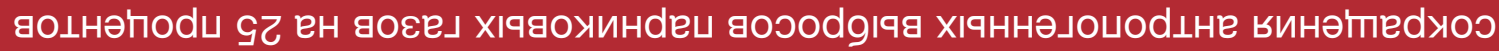

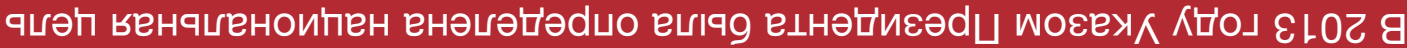

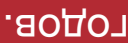

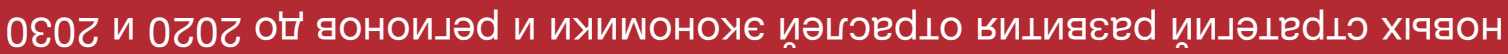

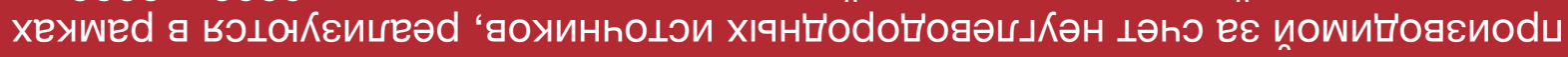

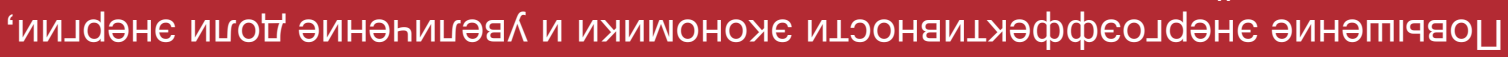

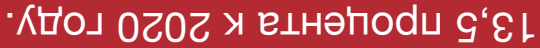

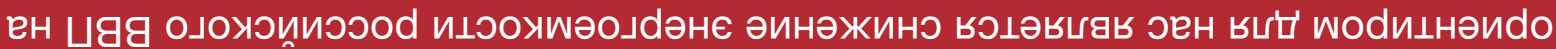

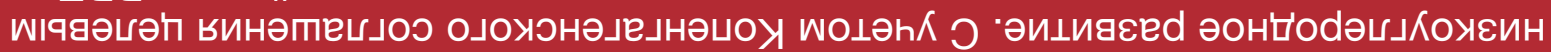

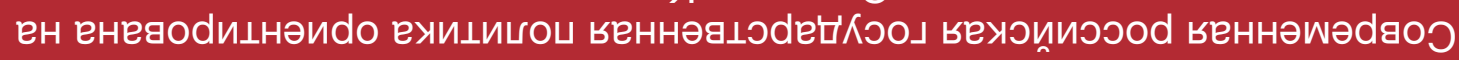

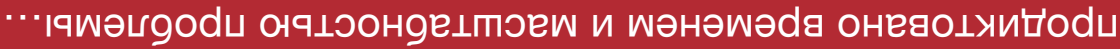

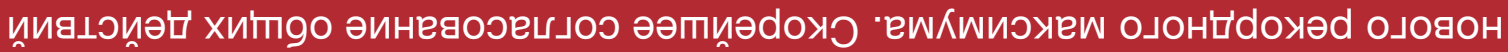

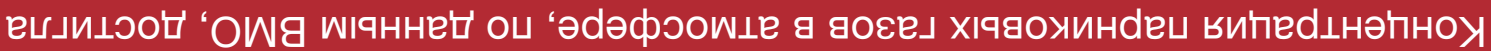

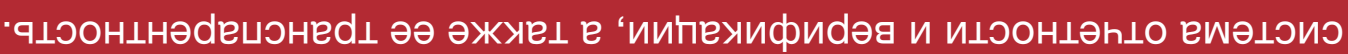

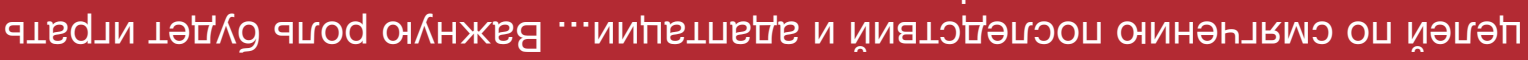

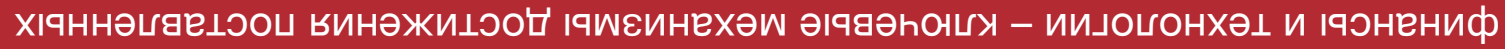

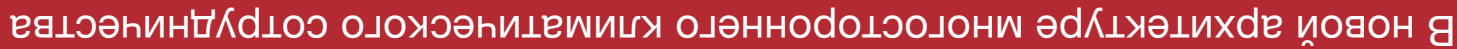

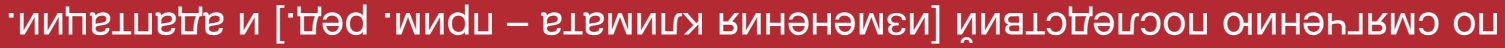

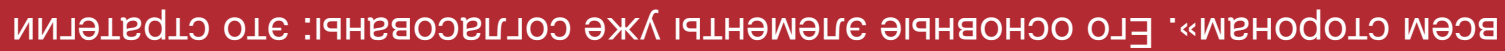

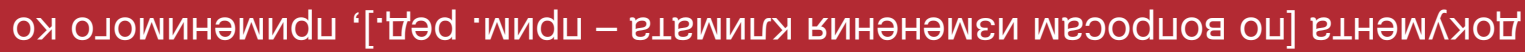

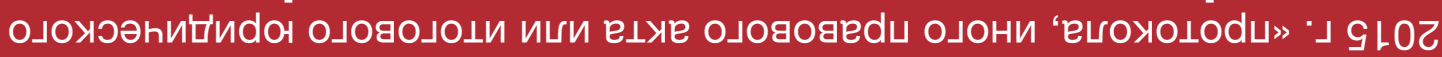

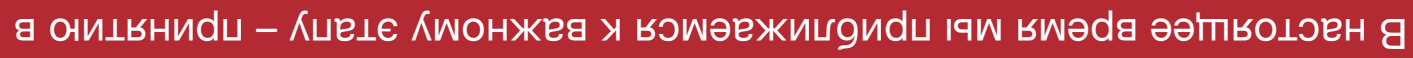

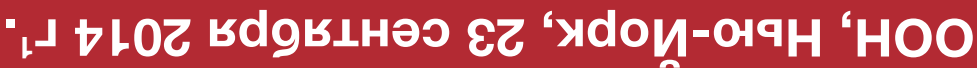

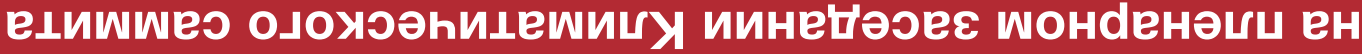

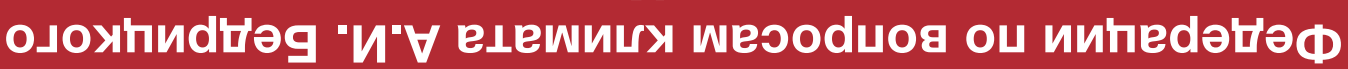

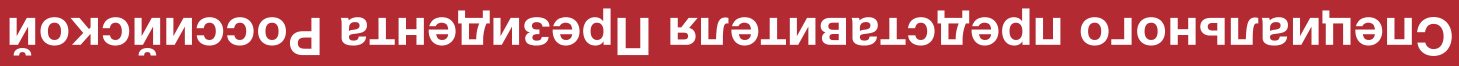

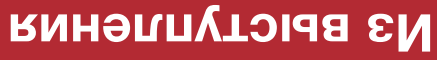




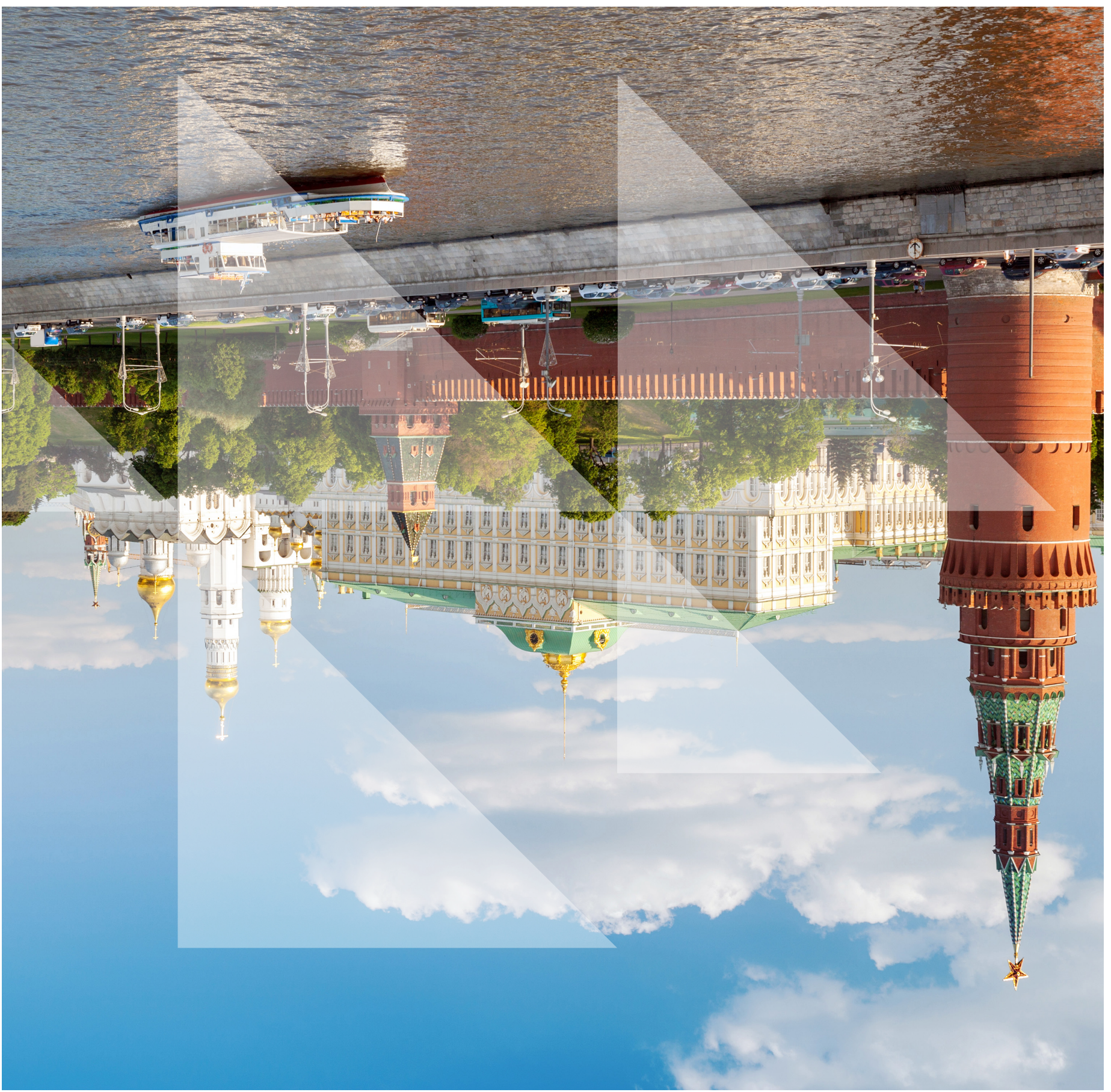

